\title{
Polycyclic Aromatic Hydrocarbon Structure Index
}

Lane C. Sander and Stephen A. Wise

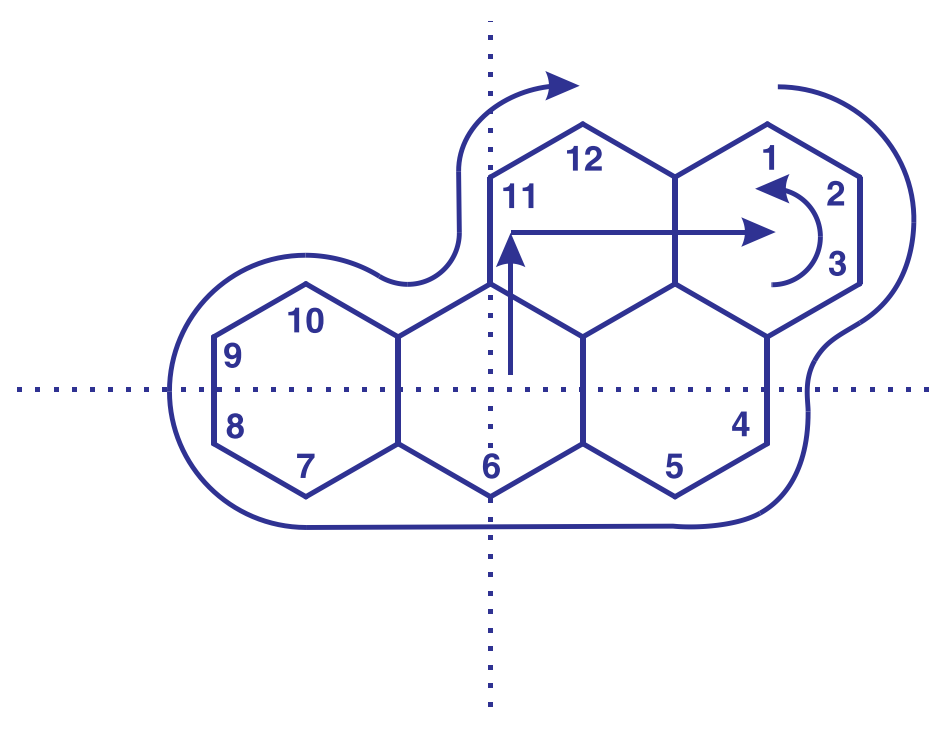

This publication is available free of charge from:

https://doi.org/10.6028/NIST.SP.922e2020 


\section{NIST Special Publication 922}

\section{Polycyclic Aromatic Hydrocarbon Structure Index}

Lane C. Sander and Stephen A. Wise

Chemical Science and Technology Laboratory National Institute of Standards and Technology

Gaithersburg, MD 20899-0001

December 1997

revised August 2020

This publication is available free of charge from:

https://doi.org/10.6028/NIST.SP.922e2020

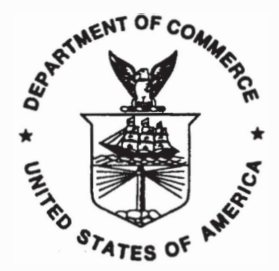

U.S. Department of Commerce Wilbur L. Ross, Jr., Secretary

National Institute of Standards and Technology

Walter Copan, NIST Director and Under Secretary of Commerce for Standards and Technology 
National Institute of Standards and Technology

Special Publication 922

Natl. Inst. Stand. Technol.

Spec. Publ. 922

105 pages (Dec. 1997)

CODEN: NSPUE2
U.S. Government Printing Office Washington: 1997

For sale by the Superintendent of Documents U.S. Government Printing Office Washington, DC 20402 


\section{Errata for SP 922 (printed document) Corrected entries are listed below}

19<smiles>C1=CC2CC3CCCC4CC(C1)CC2C43</smiles>

33<smiles>C1=CC2CCC3CCC4CCCC5CCC(C1)C2C3C54</smiles>

34<smiles>C1CC2CCC3CC4CCCC5CC(C1)C2C3C54</smiles>

41<smiles>C1CC2C(C1)C1CCCC3CCC4CCCC2C4C31</smiles>

52<smiles>C1=CC2CCC3CCC4CCCC5CCC(C1)C2C3C54</smiles>

160<smiles>C1CC2CC3CCC4CCC5CCC6CC7CCCC7C6C5C4C3CC2C1</smiles>

161<smiles>C1=CC2CC3CCC4CCC5CC6CCCC6CC5C4CC3CC2C1</smiles>

Acephenanthrylene

Cyclopenta[jkjphenanthrene

Cyclopenta[col]pyrene

Acepyrene

Acepyrylene

Benz[mno]aceanthrylene

Naphtho[1,8,7,6-cdeffluorene

1H-Cyclopenta[e]pyrene

5H-Benzo[cd]pyrene

Pyrenindene

Cyclopent $[b]$ indeno[ $[4,5-g]-$ phenanthrene

Cyclopent[b]indeno[5,6-g]phenanthrene
191-34-4

201-06-9

$\mathrm{C}_{16} \mathrm{H}_{10}$

202

1.291

11.70
9.067

3.890

27208-37-3

$\mathrm{C}_{18} \mathrm{H}_{10}$

226

1.200

11.52

9.602
3.887

203-13-4

$\mathrm{C}_{18} \mathrm{H}_{10}$

226

1.222

11.83

9.679

3.884

109587-09-9 $\quad \mathrm{C}_{19} \mathrm{H}_{12} \quad 240$

72088-81-4 $\quad \mathrm{C}_{24} \mathrm{H}_{14}$

302

72088-82-5 $\quad \mathrm{C}_{24} \mathrm{H}_{14}$

302 


\title{
Polycyclic Aromatic Hydrocarbon Structure Index
}

\author{
Lane C. Sander and Stephen A. Wise \\ Chemical Science and Technology Laboratory \\ National Institute of Standards and Technology \\ Gaithersburg, MD 20899
}

This tabulation is presented as an aid in the identification of the chemical structures of polycyclic aromatic hydrocarbons (PAHs). The Structure Index consists of two parts: (1) a cross index of named PAHs listed in alphabetical order, and (2) chemical structures including ring numbering, name(s), Chemical Abstract Service (CAS) Registry numbers, chemical formulas, molecular weights, and length-to-breadth ratios (L/B) and shape descriptors of PAHs listed in order of increasing molecular weight. Where possible, synonyms (including those employing alternate and/or obsolete naming conventions) have been included. Synonyms used in the Structure Index were compiled from a variety of sources including "Polynuclear Aromatic Hydrocarbons Nomenclature Guide," by Loening, et al. [1], "Analytical Chemistry of Polycyclic Aromatic Compounds," by Lee et al. [2], "Calculated Molecular Properties of Polycyclic Aromatic Hydrocarbons," by Hites and Simonsick [3], "Handbook of Polycyclic Hydrocarbons," by J. R. Dias [4], "The Ring Index," by Patterson and Capell [5], "CAS 12th Collective Index," [6] and "Aldrich Structure Index" [7]. In this publication the IUPAC preferred name is shown in large or bold type.

The data supplied in this Structure Index comply with IUPAC rules for naming fused ring systems, detailed in "The Nomenclature of Organic Compounds" [8] and "A Guide to IUPAC Nomenclature of Organic Compounds" [9]. Names are based on the largest fragment with a trivial name. A list of recognized compounds specified by IUPAC convention is presented in Table 1. When a choice exists, the fragment lowest in the list (i.e., with the largest number) should be used. The simplest attachments are then selected for naming. Structures are typically oriented such that 1) the greatest number of rings in a row are aligned horizontally, 2) the maximum number of rings are positioned in the upper right quadrant, and 3) the least number of rings are positioned in the lower left quadrant. Numbering begins with the uppermost ring the furthest to the right, with the most counterclockwise carbon atom not involved with ring fusion. The numbering proceeds clockwise around the structure with hydrogenated carbon atoms. The numbering of Anthracene and Phenanthrene are "retained exceptions" to this rule. In this publication, double bonds are not explicitly indicated, but aliphatic carbons are designated by associated hydrogen atoms.

The $\mathrm{L} / \mathrm{B}$ ratio is a shape-descriptive parameter that has been used in numerous studies of PAH retention in both liquid and gas chromatography [10-12]. This Structure Index represents the most comprehensive compilation of $\mathrm{L} / \mathrm{B}$ values published. $\mathrm{L} / \mathrm{B}$ values were calculated using algorithms based on the approach of Wise et al. [12]. A representation of this approach is illustrated in Figures 1 and 2. The molecular structure of each compound was generated using PC-Model and MMX molecular modeling programs (Serena Software, Bloomington, IN). ${ }^{\S}$ For planar PAHs, L/B can be determined from a two dimensional representation of the molecule. The structure is rotated

\footnotetext{
${ }^{1}$ Certain commercial equipment, instruments, or materials are identified in this report to specify adequately the experimental procedure. Such identification does not imply recommendation or endorsement by the National Institute of Standards and Technology, nor does it imply that the materials or equipment identified are necessarily the best available for the purpose.
} 
and various "trial" values for $\mathrm{L} / \mathrm{B}$ are calculated until a maximum value for $\mathrm{L} / \mathrm{B}$ is determined. Because different values for L/B will result for nonplanar molecules depending on the initial orientation, a procedure was developed to provide unambiguous orientation. The algorithm begins with an arbitrary molecular orientation. The molecule is sequentially rotated about $\mathrm{x}, \mathrm{y}$, and $\mathrm{z}$ axes and $x y, y z$, and $x z$ projections are determined with each rotation. An orientation is set such that when a box is drawn about the molecule to enclose the van der Waals surface, the minimum dimension is aligned with the $\mathrm{z}$ axis and the maximum dimension, with the $\mathrm{x}$ axis. $\mathrm{L} / \mathrm{B}$ is then calculated from the xy projection as if the molecule were planar. L/B values for planar PAHs determined using this algorithm are identical to $\mathrm{L} / \mathrm{B}$ values generated using the more simplistic planar iterative program. The dimensions of the bounding box $(\AA)$ are listed in small type below the values for L/B (dimensions $\mathrm{x}, \mathrm{y}$, and $\mathrm{z}$, respectively). A measure of solute thickness is provided by the " $z$ " box dimension. For compounds that contain only aromatic carbons, values greater than $\sim 3.9 \AA$ are indicative of nonplanarity.

Isomers are listed in order of increasing L/B for groups of the same overall ring structure. Isomers containing five-membered rings are grouped separately from isomers containing only sixmembered rings. When further distinction is possible, isomers are grouped based on the number of shared carbon atoms within the five-membered rings. For example, isomers of molecular weight (MW) 302 containing a five-membered ring with three shared carbon atoms are listed before isomers with five shared carbon atoms within the five-membered ring. MW 302 isomers containing only six-membered rings are grouped last.

The material presented is not intended to be all inclusive; however, an effort was made to include most common PAHs of environmental interest. Data was compiled from the sources provided in the reference list, and only compounds containing five- and six-membered rings are included. (Compounds were not arbitrarily drawn and named simply to enumerate all possible isomers.) Where possible, CAS numbers are supplied to facilitate literature searches. The absence of CAS numbers does not signify absence from the CAS Registry. Finally, a tabulation listing the theoretical number of possible six-membered ring configurations for various molecular weights is provided in Table 2. More detailed information on the synthesis and physical properties of many of these isomers is provided in reference [4]. The last column of this table lists the corresponding number of structures provided in this Index.

Considerable effort has been expended to make the Structure Index as accurate as possible. The authors welcome comments, corrections, and suggestions for improvements in the next revision. 
Table 1. Parent Compounds (IUPAC Convention, listed in order of increasing priority) [9]

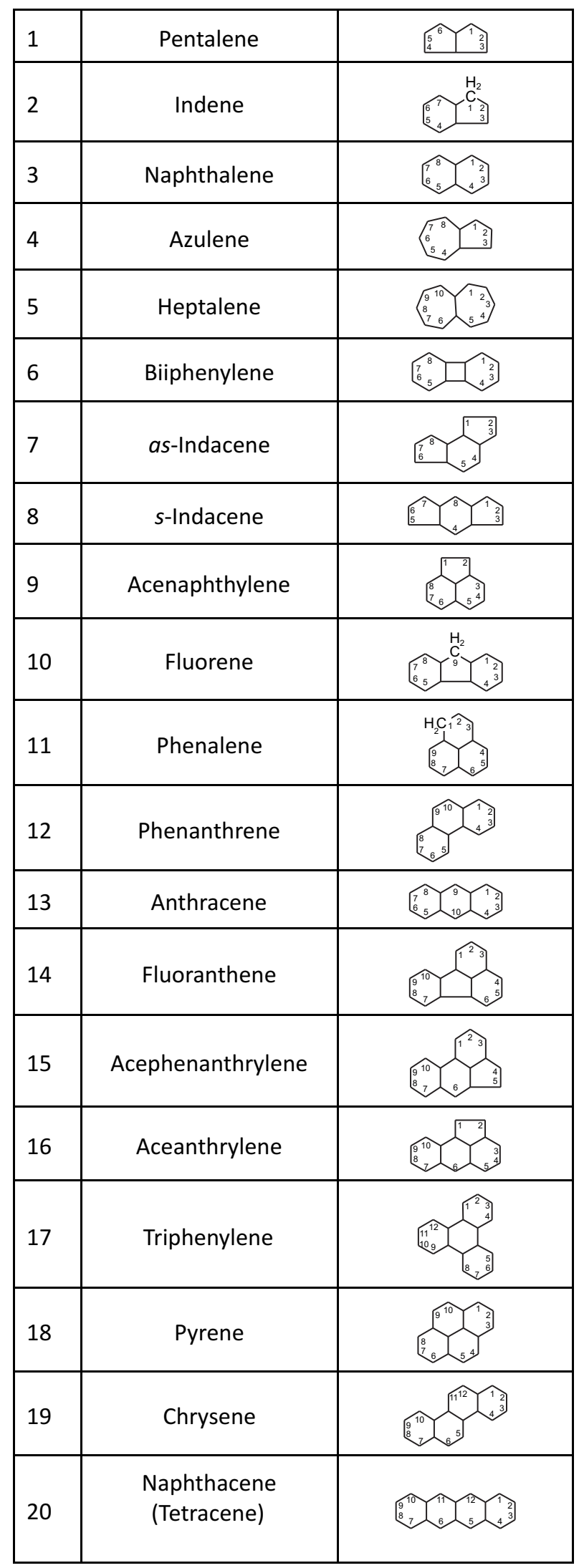

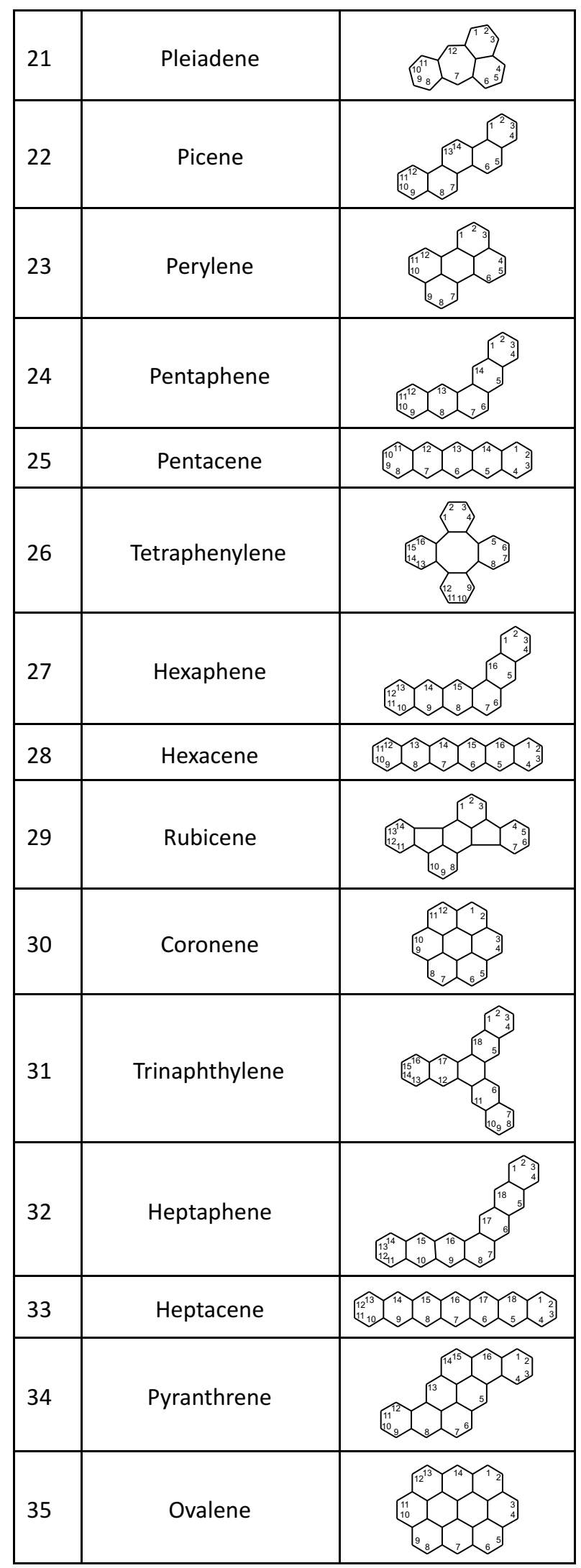


Table 2. Possible Configurations for Six Membered Ring PAH Isomers ${ }^{1}$

\begin{tabular}{|c|c|c|c|c|c|c|}
\hline MW & $\begin{array}{l}\text { Chemical } \\
\text { Formula }\end{array}$ & $\begin{array}{l}\text { Number of } \\
\text { Rings }\end{array}$ & $\begin{array}{l}\text { Internal } \\
\text { Carbons }^{2}\end{array}$ & $\begin{array}{c}\text { Isomers } \\
\text { (theoretical) }\end{array}$ & $\begin{array}{c}\text { Isomers } \\
\text { (synthesized) }\end{array}$ & $\begin{array}{l}\text { Isomers } \\
\text { (listed) }\end{array}$ \\
\hline 78 & $\mathrm{C}_{6} \mathrm{H}_{6}$ & 1 & 0 & 1 & 1 & 1 \\
\hline 128 & $\mathrm{C}_{10} \mathrm{H}_{8}$ & 2 & 0 & 1 & 1 & 1 \\
\hline 178 & $\mathrm{C}_{14} \mathrm{H}_{10}$ & 3 & 0 & 2 & 2 & 2 \\
\hline 202 & $\mathrm{C}_{16} \mathrm{H}_{10}$ & 4 & 2 & 1 & 1 & 1 \\
\hline 228 & $\mathrm{C}_{18} \mathrm{H}_{12}$ & 4 & 0 & 5 & 5 & 5 \\
\hline 252 & $\mathrm{C}_{20} \mathrm{H}_{12}$ & 5 & 2 & 3 & 3 & 3 \\
\hline 276 & $\mathrm{C}_{22} \mathrm{H}_{12}$ & 6 & 4 & 2 & 2 & 2 \\
\hline 278 & $\mathrm{C}_{22} \mathrm{H}_{14}$ & 5 & 0 & 12 & 12 & 12 \\
\hline 300 & $\mathrm{C}_{24} \mathrm{H}_{12}$ & 7 & 6 & 1 & 1 & 1 \\
\hline 302 & $\mathrm{C}_{24} \mathrm{H}_{14}$ & 6 & 2 & 13 & 13 & 13 \\
\hline 326 & $\mathrm{C}_{26} \mathrm{H}_{14}$ & 7 & 4 & 9 & 5 & 9 \\
\hline 328 & $\mathrm{C}_{26} \mathrm{H}_{16}$ & 6 & 0 & 37 & 27 & 37 \\
\hline 350 & $\mathrm{C}_{28} \mathrm{H}_{14}$ & 8 & 6 & 8 & 5 & 8 \\
\hline 352 & $\mathrm{C}_{28} \mathrm{H}_{16}$ & 7 & 2 & 62 & 24 & 45 \\
\hline 374 & $\mathrm{C}_{30} \mathrm{H}_{14}$ & 9 & 8 & 3 & 2 & 3 \\
\hline 376 & $\mathrm{C}_{30} \mathrm{H}_{16}$ & 8 & 4 & 58 & 11 & 50 \\
\hline 378 & $\mathrm{C}_{30} \mathrm{H}_{18}$ & 7 & 0 & $>123$ & 23 & 93 \\
\hline 398 & $\mathrm{C}_{32} \mathrm{H}_{14}$ & 10 & 10 & 1 & 1 & 1 \\
\hline 400 & $\mathrm{C}_{32} \mathrm{H}_{16}$ & 9 & 6 & 46 & 7 & 40 \\
\hline 402 & $\mathrm{C}_{32} \mathrm{H}_{18}$ & 8 & 2 & $>289$ & 16 & 8 \\
\hline 424 & $\mathrm{C}_{34} \mathrm{H}_{16}$ & 10 & 8 & 37 & 2 & 16 \\
\hline 426 & $\mathrm{C}_{34} \mathrm{H}_{18}$ & 9 & 4 & very large & 8 & 14 \\
\hline 428 & $\mathrm{C}_{34} \mathrm{H}_{20}$ & 8 & 0 & $>411$ & 8 & 11 \\
\hline 448 & $\mathrm{C}_{36} \mathrm{H}_{16}$ & 11 & 10 & 20 & 2 & 20 \\
\hline 450 & $\mathrm{C}_{36} \mathrm{H}_{18}$ & 10 & 6 & very large & & 9 \\
\hline 452 & $\mathrm{C}_{36} \mathrm{H}_{20}$ & 9 & 2 & very large & & 13 \\
\hline 478 & $\mathrm{C}_{38} \mathrm{H}_{22}$ & 9 & 0 & $>1489$ & 6 & 5 \\
\hline
\end{tabular}

${ }^{1}$ Data adapted from Dias, "Handbook of Polycyclic Hydrocarbons" [4]

${ }^{2}$ Refers to carbon atoms not contained in the periphery of the ring structure 


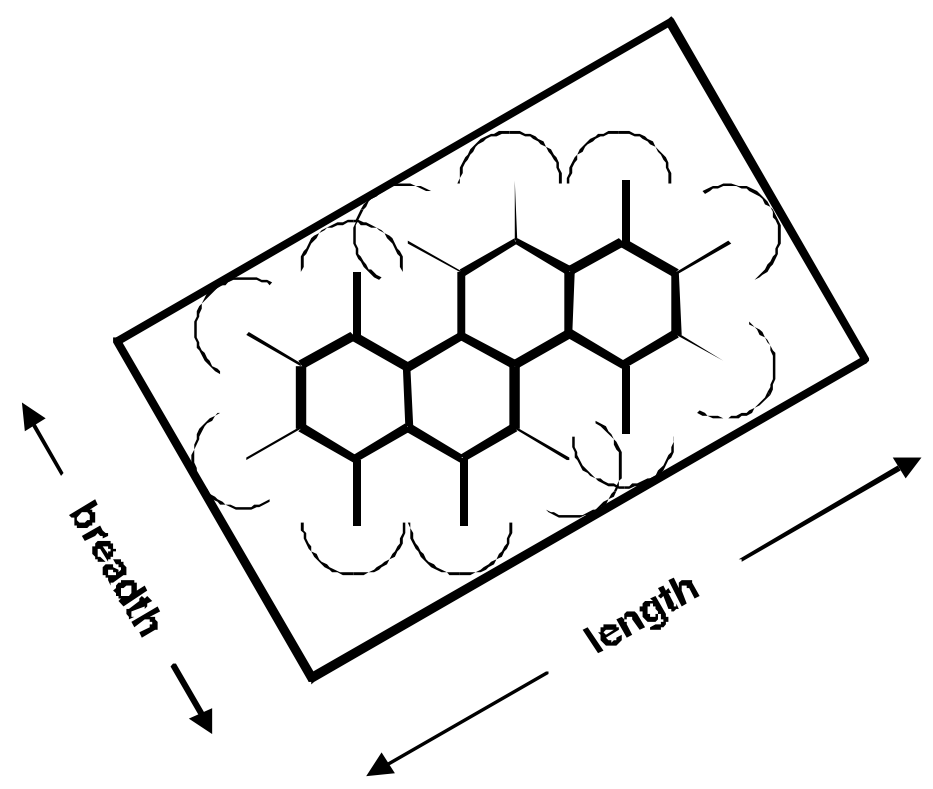

Figure 1. Depiction of Length-to-Breadth ratio for planar PAHs.

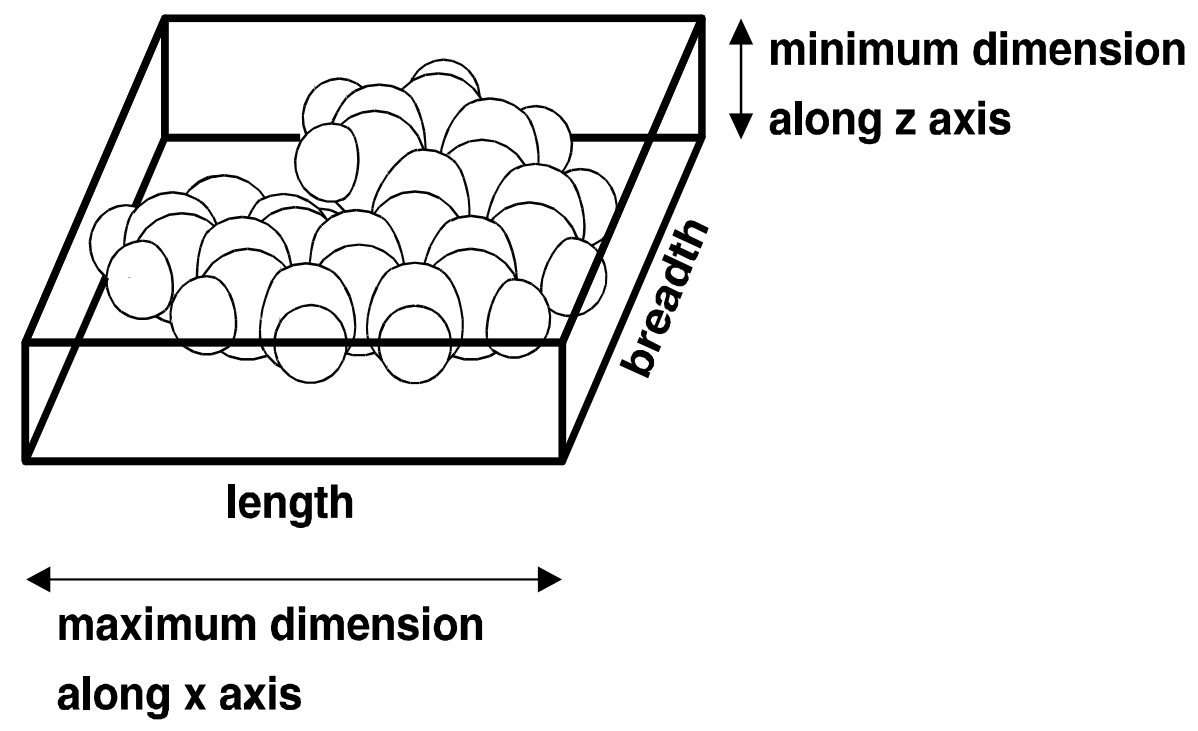

Figure 2. Depiction of Length-to-Breadth ratio algorithm for nonplanar molecules. 
Reference List

[1] Loening, K., Merritt, J., Later, D.W., and Wright, W., Polynuclear Aromatic Hydrocarbons Nomenclature Guide, Battelle Press, Columbus, 1990.

[2] Lee, M.L., Novotny, M., and Bartle, K.D., Analytical Chemistry of Polycyclic Aromatic Compounds, Academic Press, New York, 1981.

[3] Hites, R.A. and Simonsick, Jr.W.J., Calculated Molecular Properties of Polycyclic Aromatic Hydrocarbons, Elsevier, Amsterdam, 1987.

[4] Dias, J.R., Handbook of Polycyclic Hydrocarbons, Elsevier, Amsterdam, 1988.

[5] Patterson, A.M. and Capell, L.T., The Ring Index, Reinhold Publishing Corporation, New York, 1940.

[6] CAS 12th Collective Index (Compact Cambridge v C2.1), Cambridge Scientific Abstracts, Cambridge, 1994.

[7] Aldrich Structure Index, Aldrich Chemical Company, Inc., Milwaukee, 1992.

[8] Nomenclature of Organic Chemistry, 1979 Edition, Pergamon Press, Oxford, UK, 1979, pp. 20-31.

[9] A Guide to IUPAC Nomenclature of Organic Compounds, Blackwell Scientific Publications, London, 1997.

[10] Janini, G.M., Muschik, G.M., Schroer, J.A., and Zielinski, W.L., Anal.Chem., 48 (1976) 1879.

[11] Radecki, A., Lamparczyk, H., and Kaliszan, R., A Relationship Between the Retention Indices on Nematic and Isotropic Phases and the Shape of Polycyclic Aromatic Hydrocarbons, Chromatographia, 12 (1979) 595-599.

[12] Wise, S.A., Bonnett, W.J., Guenther, F.R., and May, W.E., A Relationship Between Reversed Phase C18 Liquid Chromatographic Retention and the Shape of Polycyclic Aromatic Hydrocarbons, J.Chromatogr.Sci., 19 (1981) 457-465. 


\section{Polycyclic Aromatic Hydrocarbons (Alphabetical Index)}

Aceanthrylene

Acenaphth[1,2- $a]$ anthracene

Acenaphth $[1,2-b]$ anthracene

Acenaphthalene

Acenaphthene

Acenaphtho[1,2,3-cde]pyrene

Acenaphtho[1,2-b]phenanthrene

Acenaphtho[1,2-j]fluoranthene

Acenaphtho[1,2-k]cyclopenta $[c d]$ fluoranthene

Acenaphtho[1,2-k]fluoranthene

13H-Acenaphtho[1,8-ab]phenanthrene

Acenaphthylene

Aceperylene

Acephenanthrylene

Acepyrene

Acepyrylene

[6] Annulene

Anthanthrene

Anthra[1,2,3,4-rst $]$ pentaphene

Anthra[1,2-a]aceanthrylene

Anthra[1,2-a] anthracene

Anthra [1,2-a]benz $[j]$ anthracene

Anthra[1,2-a]naphthacene

Anthra[1,2- $b]$ phenanthrene

Anthra[1,9,8-abcd $]$ benzo[hi] coronene

Anthra[2,1,9,8,7-defghi]benzo[op]pentacene

Anthra[2,1,9,8,7-defghi] benzo[st]pentacene

Anthra[2,1,9,8,7-defghi $]$ benzo[uv]pentacene

Anthra[2,1,9,8-defgh $]$ benzo[rst]pentaphene

Anthra[2,1,9,8-defgh]pentaphene

Anthra[2,1,9,8-opqra]naphthacene

Anthra[2,1,9,8-stuva]pentacene

Anthra[2,1,9-qra]naphthacene

Anthra[2,1-a]aceanthrylene

Anthra[2,1-a]naphthacene

Anthra [2,3- $a]$ coronene

Anthra[2,3-a]naphthacene

Anthra[3,2,1,9,8-rstuva]benzo[ij]pentaphene

Anthra[3,2,1,9-pqra $]$ benzo $[c d]$ perylene

Anthra[7,8,9,1,2,3-rstuvwx] hexaphene

Anthra $[8,9,1,2$-cdefg $]$ benzo $[a]$ naphthacene

Anthra [8,9,1,2-lmnop]benzo[ $a]$ naphthacene

Anthra $[9,1,2-a b c]$ coronene

Anthra $[9,1,2-b c d]$ perylene

Anthra $[9,1,2-c d e]$ benzo[rst $]$ pentaphene

Anthra[9,1-bc]fluorene

Anthracene

Anthraceno[2,1- $a]$ anthracene
Anthraceno-1',2',1,2-anthracene 277

Anthracin 15

Anthrodianthrene 374

Benz $[4,10]$ anthra $[1,9,8-a b c d]$ coronene $\quad 625$

$15 H$-Benz $[4,5]$ indeno[1,2-l]phenanthrene 246

9H-Benz [4,5]indeno[2,1-c]phenanthrene 249

7H-Benz [5,6]indeno[1,2-a]phenanthrene 251

Benz $[5,6]$ indeno[2,1- $a]$ phenalene 179

7H-Benz $[5,6]$ indeno[2,1-a]phenanthrene 252

9H-Benz[5,6]indeno[2,1-c]phenanthrene 245

Benz $[a]$ aceanthrylene $\quad 68$

Benz $[a]$ acenaphthylene 18

Benz $[a]$ acephenanthrylene $\quad 59$

1,2:5,6-Benz $[a]$ anthracene $\quad 130$

Benz $[a]$ anthracene 38

Benz $[a]$ indeno[1,2-c]fluorene $\quad 176$

Benz $[a]$ indeno $[2,1-c]$ naphthalene $\quad 165$

Benz $[a]$ indeno[5,6-g]fluorene 174

Benz $[a]$ ovalene

Benz $[b]$ anthracene 40

Benz $[b]$ indeno[2,1- $h]$ fluorene $\quad 170$

11H-Benz $[b c]$ aceanthrylene $\quad 47$

Benz $[c]$ indeno[2,1-a]fluorene 166

Benz $[d]$ aceanthrylene $\quad 67$

Benz $[d]$ ovalene

1H-Benz $[d e]$ anthracene 32

Benz $[d e]$ indeno[2,1-b]anthracene $\quad 178$

Benz $[$ def $]$ indeno $[1,2,3-h i]$ chrysene $\quad 260$

Benz $[$ def $]$ indeno $[1,2,3-q r]$ chrysene 259

Benz $[e]$ aceanthrylene 61

3,4-Benz[e]acephenanthrylene 69

Benz $[e]$ acephenanthrylene

$1 H$-Benz $[e]$ indene 10

$3 H$-Benz $[e]$ indene

$1 H$-Benz $[f]$ indene

$1 H$-Benz $[f g]$ aceanthrylene

$5 H$-Benz $[f g]$ acenaphthylene $\quad 16$

$10 H$-Benz $[g]$ indeno $[2,1-a]$ phenanthrene $\quad 247$

Benz[j]aceanthrylene $\quad 64$

Benz[j]acephenanthrylene 63

Benz $[k]$ acephenanthrylene $\quad 65$

Benz $[l]$ aceanthrylene $\quad 62$

Benz $[l]$ acephenanthrylene $\quad 60$

Benz $[m n o]$ aceanthrylene $\quad 34$

Benz $[$ mno $]$ indeno $[1,7,6,5-c d e f]$ chrysene $\quad 157$

Benz [mno]indeno[5,6,7,1-defg $]$ chrysene $\quad 154$

4,5-Benz-10,11-(1',2'-naphtha)chrysene 518

1,2:5,6-Benzanthracene 130 
1,2-Benzanthracene

2,3-Benzanthracene

1,2-Benzanthrene

$1 H$-meso-Benzanthrene

Benzanthrene

Benzanthreno- $B z-1, B z-2: 2,3$-naphthalene

Benzene

9,10[1',2']-Benzenoanthracene, 9,10-dihydro

7,8-Benzfluoranthene

2,3-Benzidene

Benzine

Benzo[1,2- $\left.a, 3,4-a^{\prime}, 5,6-a^{\prime}\right]$ triacenaphthylene

Benzo[1,2- $\left.a, 4,5-a^{\prime}\right]$ diacenaphthylene

Benzo[2,1-a:3,4-a']dianthracene

Benzo[3,4]phenanthro[2,1,10,9,8,7-pqrstuv]pentaphene

Benzo[6,7]phenanthro[4,3-b]chrysene

Benzo $[a]$ anthanthrene

Benzo $[a]$ coronene

$1 H$-Benzo $[a]$ cyclopent $[h]$ anthracene

9H-Benzo $[a]$ cyclopent $[i]$ anthracene

Benzo $[a]$ cyclopenta $[d e]$ naphthacene

Benzo $[a]$ cyclopenta $[f g]$ naphthacene

Benzo $[a]$ cyclopenta $[h i]$ naphthacene

Benzo $[a]$ cyclopenta $[m n]$ naphthacene

Benzo $[a]$ cyclopenta $[o p]$ naphthacene

Benzo $[a]$ fluoranthene

$11 H$-Benzo[a]fluorene

Benzo $[a]$ fluorene

Benzo $[a]$ heptacene

Benzo $[a]$ hexacene

Benzo $[a]$ hexaphene

Benzo $[a]$ naphth $[2,1-j]$ anthracene

Benzo $[a]$ naphthacene

Benzo $[a]$ naphtho[1,2,3,4-ghi]perylene

Benzo[ $[a]$ naphtho[1,2-c]naphthacene

Benzo $[a]$ naphtho[1,2- $h]$ anthracene

Benzo $[a]$ naphtho[1,2-j]naphthacene

Benzo $[a]$ naphtho[1,2-l] naphthacene

Benzo[a]naphtho[2,1,8-cde]perylene

Benzo $[a]$ naphtho[2,1,8-hij]naphthacene

Benzo $[a]$ naphtho[2,1,8-lmn]perylene

Benzo $[a]$ naphtho[2,1- $h]$ pyrene

Benzo $[a]$ naphtho[2,1-j]naphthacene

Benzo $[a]$ naphtho[2,1-l]naphthacene

Benzo[a]naphtho[7,8,1,2,3-pqrst]pentaphene

Benzo $[a]$ naphtho $[8,1,2-c d e]$ naphthacene

Benzo $[a]$ naphtho $[8,1,2-k l m]$ perylene

Benzo $[a]$ naphtho $[8,1,2-$ Imn $]$ naphthacene

Benzo $[a]$ pentacene

Benzo $[a]$ pentaphene

Benzo $[a]$ perylene

Benzo $[a]$ phenanthrene

Benzo $[a]$ picene
Benzo $[a]$ pyranthrene $\quad \mathbf{5 8 8}$

Benzo[a]pyrene $\quad 74$

Benzo $[b]$ anthanthrene $\quad 269$

Benzo $[b]$ chrysene $\quad 131$

5H-Benzo $[b]$ cyclopenta $[$ def $]$ chrysene $\quad 147$

$13 H$-Benzo $[b]$ cyclopenta $[d e f]$ triphenylene $\quad 140$

Benzo $[b]$ cyclopenta $[h i]$ chrysene $\quad 204$

$4 H$-Benzo[ $[b]$ cyclopenta $[j k l]$ triphenylene $\quad 139$

4H-Benzo[b]cyclopenta[mno]chrysene $\quad 149$

Benzo $[b]$ cyclopenta $[q r]$ chrysene $\quad 197$

Benzo[b]fluoranthene $\quad 69$

11 $H$-Benzo[b]fluorene $\quad 31$

Benzo[b]naphthacene 133

Benzo $[b]$ naphtho[1,2,3,4-pqr]perylene 382

Benzo $[b]$ naphtho $[1,2-k]$ chrysene $\quad 518$

Benzo $[b]$ naphtho[1,2-l]chrysene 499

Benzo $[b]$ naphtho[2,1-g]chrysene 449

Benzo $[b]$ naphtho[2,1-k]chrysene $\quad 502$

Benzo $[b]$ naphtho[2,1-p]chrysene 440

Benzo $[b]$ naphtho[2,3-g]chrysene 439

Benzo[b]naphtho[2,3-j]chrysene 508

Benzo $[b]$ naphtho[2,3-l]chrysene 478

Benzo $[b]$ naphtho[8,1,2-pqr $]$ chrysene 343

Benzo $[b]$ pentahelicene 274

Benzo $[b]$ pentaphene 298

Benzo $[b]$ perylene $\quad 238$

Benzo $[b]$ phenanthrene 38

Benzo $[b]$ picene $\quad 302$

Benzo $[b]$ triphenylene $\quad 123$

Benzo $[b c]$ naphtho[1,2,3-ef]coronene $\quad 577$

Benzo[bc]naphtho[3,2,1-ef]coronene $\quad 574$

Benzo $[c]$ chrysene $\quad 126$

Benzo $[c]$ cyclopenta $[h i]$ chrysene $\quad 190$

4H-Benzo $[c]$ cyclopenta $[$ mno $]$ chrysene $\quad 143$

Benzo[c]cyclopenta $[q r]$ chrysene $\quad 187$

7H-Benzo[c]fluorene 29

Benzo[c]fluorene $\quad 29$

Benzo $[c]$ hexaphene $\quad 506$

Benzo $[c]$ naphtho[1,2-l]chrysene $\quad 495$

Benzo $[c]$ naphtho $[2,1-m]$ pentaphene $\quad 608$

Benzo $[c]$ naphtho[2,1-p]chrysene $\quad 429$

Benzo[c]naphtho[2,3-l]chrysene 477

Benzo[c]naphtho[7,8,1,2,3-pqrst]pentaphene 419

Benzo $[c]$ naphtho $[8,1,2-$ ghi $]$ chrysene $\quad 333$

Benzo[c]pentahelicene 278

Benzo[c]pentaphene 299

Benzo[c]phenanthrene $\quad 37$

Benzo $[c]$ picene $\quad 306$

Benzo[c]tetraphene 131

$1 H$-Benzo $[c d]$ fluoranthene $\quad 45$

Benzo $[c d]$ naphtho[3,2,1,8-pqra]perylene $\quad 314$

$2 H$-Benzo[cd]pyrene $\quad 50$

$3 H$-Benzo[cd]pyrene $\quad 49$ 
$5 H$-Benzo $[c d]$ pyrene

6H-Benzo $[c d]$ pyrene

Benzo $[d e]$ cyclopent $[a]$ anthracene

Benzo[de]cyclopent $[b]$ anthracene

Benzo[de]naphtho[2,1,8,7-qrst $]$ pentacene

Benzo[de]naphtho[3,2,1-mn]naphthacene

Benzo[de]naphtho[8,1,2,3-stuv]picene

7H-Benzo[de]pentacene

Benzo[def $]$ chrysene

Benzo[def]cyclopenta[hi]chrysene

4H-Benzo[def]cyclopenta[mno]chrysene

Benzo[def $]$ cyclopenta $[q r]$ chrysene

Benzo[def]fluorene

Benzo[def]phenanthrene

Benzo[def]pyranthrene

Benzo[e]anthanthrene

Benzo[e]cyclopenta $[j k]$ pyrene

Benzo[e]cyclopenta $[j k]$ pyrene

Benzo[e]fluoranthene

Benzo[e]phenanthro[1,10,9,8-opqra]perylene

Benzo[e]phenanthro[2,3,4,5-pqrab]perylene

Benzo[e]pyrene

Benzo[ef $]$ phenaleno $[9,1,2-a b c]$ coronene

Benzo[f]pentahelicene

Benzo[f]picene

Benzo $[f g]$ cyclopent $[a]$ anthracene

Benzo $[f g]$ naphtho[1,2,3-op $]$ naphthacene

Benzo $[g]$ chrysene

8H-Benzo $[g]$ cyclopenta $[m n o]$ chysene

Benzo $[g]$ naphtho[2,1-b]chrysene

Benzo $[g]$ naphtho $[8,1,2-a b c]$ coronene

Benzo $[g h i]$ cyclopenta $[c d]$ perylene

$1 H$-Benzo[ghi]cyclopenta $[p q r]$ perylene

Benzo[ghi]fluoranthene

Benzo[ghi]naphtho[2,1-a]perylene

Benzo[ghi]naphtho[2,1-b]perylene

Benzo[ghi]perylene

Benzo $[h]$ naphtho[1,2,3,4-rst $]$ pentaphene

Benzo[h]naphtho[7,8,1,2,3-pqrst $]$ pentaphene

Benzo $[h]$ pentaphene

Benzo[h]phenanthro[2,1,10,9,8,7-pqrstuv]pentaphene

4H-Benzo[hi]chrysene

7H-Benzo[hi]chrysene

Benzo[i]pentahelicene

Benzo[ij] naphtho[2,1,8,7-defg]pentaphene

Benzo[j]benzo[2,1-a:3,4- $\left.a^{\prime}\right]$ dianthracene

Benzo[j]fluoranthene

Benzo[j]naphtho[8,1,2-abc]coronene

Benzo[jk]fluorene

Benzo $[k]$ fluoranthene

Benzo $[k l]$ naphtho $[2,1,8,7-d e f g]$ pentaphene

Benzo $[l]$ cyclopenta[ $[c d]$ pyrene

Benzo[l]fluoranthene
Benzo $[l]$ naphtho[1,2-b]chrysene 494 Benzo $[l]$ naphtho[2,1-b]chrysene 496 Benzo[l]phenanthrene 36

Benzo $[I m]$ naphtho[1,8-ab]perylene $\quad 394$

Benzo $[l m]$ phenanthro $[5,4,3-a b c d]$ perylene $\quad 545$

Benzo[Imn]naphtho[2,1,8-qra]perylene 317

Benzo $[m]$ naphtho $[8,1,2-a b c]$ coronene $\quad 582$

Benzo[mno]fluoranthene 35

Benzo[mno]naphtho[1,2-c]chrysene $\quad 359$

Benzo[mno]naphtho[2,1-c]chrysene 332

Benzo[o]hexaphene $\quad \mathbf{5 0 5}$

$8 H$-Benzo[ $p]$ cyclopenta[def]chrysene 138

Benzo $[p]$ hexaphene 498

Benzo $[p]$ naphtho[1,2-b]chrysene $\quad 470$

Benzo $[p]$ naphtho $[1,8,7-$ ghi $]$ chrysene $\quad 327$

Benzo $[p]$ naphtho $[2,1-b]$ chrysene $\quad 469$

Benzo $[p]$ naphtho $[8,1,2-a b c]$ coronene $\quad 572$

Benzo $[p q r]$ dinaphtho[8,1,2-bcd:2',1',8'-lmn]perylene $\quad 585$

Benzo[pqr]naphtho[1,2-b]perylene 393

Benzo[pqr]naphtho[2,1,8-def]picene 406

Benzo[pqr]naphtho[2,1-b]perylene 410

Benzo[pqr]naphtho[8,1,2-bcd]perylene 318

Benzo[pqr]naphtho[8,1,2-cde]picene $\quad 427$

Benzo[pqr]picene $\quad 242$

Benzo $[q]$ hexaphene $\quad 476$

Benzo $[q r]$ naphtho[2,1,8,7-defg $]$ pentacene $\quad 416$

Benzo[qr]naphtho[2,1,8,7-fghi]pentacene 409

Benzo[qr]naphtho[3,2,1,8-defg]chrysene 264

Benzo[qrs]naphtho[3,2,1,8,7-defgh]pyranthrene $\quad 632$

Benzo[rst $]$ dinaphtho $\left[8,1,2-c d e: 2^{\prime}, 1^{\prime}, 8^{\prime}-k l m\right]$ pentaphene $\quad 642$

Benzo $[r s t]$ naphtho[2,1,8-fgh $]$ pentaphene $\quad 395$

Benzo[rst $]$ naphtho $[8,1,2-c d e]$ pentaphene $\quad 428$

Benzo[rst $]$ pentaphene 243

Benzo[rst $]$ phenanthro[1,10,9-cde]pentaphene $\quad 598$

Benzo[rst $]$ phenanthro[10,1,2-cde]pentaphene 599

Benzo[rst $]$ pyreno[1,10,9-cde]pentaphene 639

Benzo[s]picene 288

Benzo[st]naphtho[2,1,8,7-defg $]$ pentacene 408

Benzo[tuv]naphtho[2,1-b]picene 571

Benzo[uv]naphtho[2,1,8,7-defg]pentacene $\quad 405$

Benzo[uv]naphtho[2,1,8,7-defg]pentaphene 397

Benzo[vwx]hexaphene $\quad 361$

1,10-(peri)-Benzo-1,5-dihydropyrene 49

2,3-Benzo-6,7-naphthoanthracene 307

1,2-Benzoacenaphthylene 18

Benzobenzanthrene 102

2,3-Benzochrysene 131

15,16-Benzodehydrocholanthrene 222

o-meso-Benzodianthrene $\quad 324$

p-meso-Benzodianthrene 337

10,11-Benzofluoranthene 70

11,12-Benzofluoranthene $\quad 71$ 
2,13-Benzofluoranthene

2,3-Benzofluoranthene

3,4-Benzofluoranthene

7,10-Benzofluoranthene

8,9-Benzofluoranthene

Benzofluoranthene

1,2-Benzofluorene

2,3-Benzofluorene

3,4-Benzofluorene

Benzol

$1 H$-Benzonaphthene

1,12-Benzoperylene

1,2-Benzoperylene

2,3-Benzoperylene

1,2-Benzophenanthrene

2,3-Benzophenanthrene

3,4-Benzophenanthrene

9,10-Benzophenanthrene

2,3-Benzopicene

3,4-Benzopyrene

6,7-Benzopyrene

3,4-Benzotetraphene

1,2-Benzperylene

1,2-Benzpyrene

4,5-Benzpyrene

2,3-Benztriphenylene

2,3:1',8'-Binaphthylene

3,4-(o,o'-Biphenylene)cyclopentadiene

3,4-(o,o'-Biphenylene)fluorene

o-Biphenylenemethane

Biphenylenephenanthrene

o-Biphenylmethane

Bisanthrene

Ceranthrene

homeo-Cerodianthrene

Cholanthrene

Cholanthrylene

Chrysene

Chryseno[2,1-b]picene

Chrysofluorene

Corannulene

Coronene

1,2-Cyclo- $\Delta^{1,33^{\prime}}$-pentadienophenanthrene

1,2-Cyclo- $\Delta^{1,4^{\prime}}$-pentadienophenanthrene

Cyclohexatriene

$1 H$-Cyclopent $[a]$ anthracene

$1 H$-Cyclopent $[b]$ anthracene

Cyclopent $[b]$ indeno $[4,5-g]$ phenanthrene

Cyclopent $[b]$ indeno $[5,6-g]$ phenanthrene

Cyclopent $[i]$ indeno $[5,6-a]$ anthracene

$15 H$-Cyclopenta $[a]$ phenanthrene

$17 H$-Cyclopenta $[a]$ phenanthrene

$1 H$-Cyclopenta $[a]$ pyrene
$11 H$-Cyclopenta $[a]$ triphenylene $\quad 80$

8H-Cyclopenta $[b]$ phenanthrene $\quad 24$

Cyclopenta $[c d]$ perylene $\quad 110$

Cyclopenta $[c d]$ pyrene $\quad 33$

Cyclopenta[de]anthracene 20

Cyclopenta $[d e]$ naphthacene $\quad 66$

Cyclopenta[de]naphthalene 7

Cyclopenta $[d e]$ pentacene $\quad 210$

Cyclopenta[de]pentaphene $\quad 202$

Cyclopenta $[d e]$ picene $\quad 205$

4H-Cyclopenta $[$ def $]$ chrysene $\quad 48$

4H-Cyclopenta $[$ def $]$ phenanthrene $\quad 17$

4H-Cyclopenta $[d e f]$ triphenylene $\quad 46$

$1 H$-Cyclopenta $[e]$ pyrene $\quad 41$

Cyclopenta[ $[g]$ naphthacene $\quad 67$

Cyclopenta $[f g]$ pentacene $\quad 209$

Cyclopenta[f $g]$ pentaphene $\quad 192$

$11 H$-Cyclopenta $[g h i]$ perylene $\quad 78$

6H-Cyclopenta $[$ ghi $]$ picene $\quad 150$

Cyclopenta $[h i]$ chrysene $\quad 63$

Cyclopenta[jk]phenanthrene $\quad 19$

1H-Cyclopenta $[l]$ phenanthrene 22

$2 H$-Cyclopenta $[l]$ phenanthrene 23

Cyclopenta[pq]pentaphene $\quad 193$

13H-Cyclopenta[pqr]picene $\quad 148$

13H-Cyclopenta $[r s t]$ pentaphene $\quad 144$

Cyclopentaphenanthrene $\quad 17$

Decayclene $\quad 633$

Dehydro-8,9-trimethylene-1,2-benzanthracene 104

3,4,1,6-Di(1,8-naphthylene)benzene 257

1,9,5,10-Di(peri-naphthylene)anthracene 596

Di- $\beta$-naphthofluorene $\quad 96$

Dibenz $[a, c]$ anthracene $\quad 123$

Dibenz $[a, e]$ aceanthrylene $\quad 214$

Dibenz $[a, e]$ acephenanthrylene $\quad 212$

Dibenz $[a, h]$ anthracene $\quad 130$

Dibenz $[a, j]$ aceanthrylene $\quad 222$

Dibenz $[a, j]$ anthracene $\quad 127$

Dibenz $[a, k]$ acephenanthrylene $\quad 183$

$1 H$-Dibenz $[a, k l]$ anthracene $\quad 104$

$4 H$-Dibenz $[a, k l]$ anthracene $\quad 103$

7H-Dibenz $[a, k l]$ anthracene $\quad 102$

Dibenz $[a, l]$ aceanthrylene $\quad 215$

Dibenz $[a, n]$ triphenylene $\quad 273$

$13 H$-Dibenz $[b c, j]$ aceanthrylene $\quad 146$

13H-Dibenz $[b c, l]$ aceanthrylene $\quad 142$

Dibenz[de,kl]anthracene $\quad 73$

Dibenz $[e$, ghi $]$ indeno[1,2,3,4-pqra $]$ perylene $\quad 372$

Dibenz $[e, j]$ aceanthrylene $\quad 188$

Dibenz $[e, k]$ acephenanthrylene $\quad 228$

Dibenz $[e, l]$ aceanthrylene $\quad 195$

Dibenz[e,l]acephenanthrylene 224

1,2,6,7-Dibenzanthracene $\quad 129$ 
1,2,7,8-Dibenzanthracene

1,2:3,4-Dibenzanthracene

2,3:6,7-Dibenzanthracene

3,4,5,6-Dibenzanthracene

$\beta, \beta$ '-Dibenzanthracene

1,2,3,4-Dibenznaphthalene

Dibenzo $[a, c]$ chrysene

13H-Dibenzo[ $[a, c]$ fluorene

Dibenzo[a,c]naphthacene

Dibenzo $[a, c]$ pentacene

Dibenzo[a,c]pentaphene

Dibenzo[a,c]picene

Dibenzo $[a, c]$ tetraphene

Dibenzo $[a, c]$ triphenylene

Dibenzo[ $[a, c d]$ naphtho $[8,1,2,3-f g h i]$ perylene

Dibenzo $[a, d]$ coronene

13H-Dibenzo[ $a, d e]$ naphth $[2,3-h]$ anthracene

$4 H$-Dibenzo[a,de]naphthacene

4H-Dibenzo $[a, d e]$ pentacene

Dibenzo $[a, e]$ fluoranthene

Dibenzo[a,e]pyrene

Dibenzo $[a, f]$ fluoranthene

Dibenzo $[a, f]$ perylene

Dibenzo $[a, f]$ picene

Dibenzo $[a, f]$ tetraphene

Dibenzo[a,g]coronene

13H-Dibenzo $[a, g]$ fluorene

Dibenzo[a,ghi] naphtho[2,1,8-cde]perylene

Dibenzo[ $[a, g h i]$ naphtho[2,1,8-lmn]perylene

Dibenzo $[a, g h i]$ naphtho $[8,1,2-k l m]$ perylene

Dibenzo $[a, g h i]$ perylene

13H-Dibenzo[a,h]fluorene

Dibenzo $[a, h]$ pentaphene

Dibenzo $[a, h]$ phenanthrene

Dibenzo $[a, h]$ pyrene

13H-Dibenzo $[a, i]$ fluorene

Dibenzo $[a, i]$ pyrene

Dibenzo $[a, j]$ coronene

Dibenzo $[a, j]$ fluoranthene

Dibenzo $[a, j]$ naphthacene

Dibenzo $[a, j]$ perylene

Dibenzo $[a, j]$ plcene

Dibenzo $[a, j]$ tetracene

Dibenzo $[a, j k]$ fluorene

Dibenzo $[a, k]$ fluoranthene

Dibenzo $[a, k]$ tetraphene

Dibenzo $[a, l]$ fluoranthene

Dibenzo $[a, l]$ naphthacene

Dibenzo[ $[a, l]$ pentacene

Dibenzo[a,l]pyrene

Dibenzo $[a, m]$ pentaphene

Dibenzo $[a, m]$ tetraphene

Dibenzo $[a, n]$ pentacene
Dibenzo[a,n]perylene

Dibenzo $[a, o]$ pentaphene $\quad 451$

Dibenzo $[a, o]$ perylene $\quad 324$

Dibenzo $[a, o]$ picene $\quad 463$

Dibenzo[a,p]chrysene 288

Dibenzo[a,pqr]picene 355

Dibenzo[a,rst $]$ naphtho[8,1,2-cde $]$ pentaphene $\quad 597$

Dibenzo $[a, r s t]$ pentaphene $\quad 348$

Dibenzo[b,def]chrysene $\quad 244$

Dibenzo[b,e]fluoranthene 212

Dibenzo $[b, f]$ picene $\quad 466$

$8 H$-Dibenzo $[b, f g]$ pyrene $\quad 151$

Dibenzo[b,g]chrysene 286

$\mathbf{7 H}$-Dibenzo $[\boldsymbol{b}, \boldsymbol{g}]$ fluorene $\quad 88$

Dibenzo $[b, g]$ phenanthrene $\quad 125$

Dibenzo[b,ghi]fluoranthene $\quad 119$

Dibenzo[b,ghi]perylene 265

12H-Dibenzo[b,h]fluorene 96

Dibenzo[$[b, h]$ phenanthrene $\quad 128$

Dibenzo[b, $h]$ pyrene 243

Dibenzo[b,j]fluoranthene $\quad 227$

Dibenzo $[b, j]$ plcene $\quad 479$

Dibenzo[b,jk]fluorene $\quad 71$

Dibenzo $[\boldsymbol{b}, \boldsymbol{k}]$ chrysene $\quad 305$

Dibenzo[b,k]fluoranthene 225

Dibenzo[b,k]perylene 356

Dibenzo $[b, l]$ chrysene 296

Dibenzo[b,l]fluoranthene 220

Dibenzo $[b, m]$ picene $\quad 516$

13H-Dibenzo[b,mn]phenanthrene $\quad 101$

8H-Dibenzo $[b, m n]$ phenanthrene $\quad 100$

Dibenzo[b,mno]fluoranthene 118

Dibenzo $[b, n]$ pentaphene 497

Dibenzo $[b, n]$ perylene 352

Dibenzo $[b, n]$ picene $\quad 508$

Dibenzo $[b, p]$ chrysene $\quad 284$

Dibenzo[b,pqr]perylene $\quad 266$

Dibenzo $[b, q r]$ naphtho[3,2,1,8-defg $]$ chrysene $\quad 401$

Dibenzo $[b, s]$ picene $\quad 481$

Dibenzo[b,tuv $]$ naphtho[2,1-m]picene $\quad 655$

Dibenzo $[b, t u v]$ picene $\quad 368$

Dibenzo[bc,ef]coronene $\quad 374$

Dibenzo $[b c, k l]$ coronene $\quad 376$

Dibenzo $[c, f]$ tetraphene 284

Dibenzo $[c, g]$ chrysene $\quad 280$

$7 \boldsymbol{H}$-Dibenzo $[c, g]$ fluorene $\quad 85$

Dibenzo[c,g]phenanthrene $\quad 122$

Dibenzo $[c, h]$ pentaphene $\quad 462$

Dibenzo[c,hi] naphtho[3,2,1,8-mnop]chrysene 381

Dibenzo[c,i]cyclopenta $[a]$ fluorene $\quad 162$

Dibenzo[c,k]tetraphene 304

Dibenzo $[c, l]$ chrysene 293 
Dibenzo $[c, l m]$ fluorene

Dibenzo $[c, m]$ pentaphene

Dibenzo[c,m]picene

Dibenzo $[c, m]$ tetraphene

5H-Dibenzo $[c, m n]$ phenanthrene

Dibenzo[c,mno]chrysene

Dibenzo $[c, p]$ chrysene

Dibenzo[c,pqr]picene

Dibenzo $[c, r s t]$ pentaphene

Dibenzo[c,s]picene

Dibenzo $[c d, f g]$ anthanthrene

Dibenzo $[c d, h i]$ anthanthrene

Dibenzo $[c d, j k]$ pyrene

Dibenzo[cd,k]naphtho[3,2,1,8-pqra]perylene

Dibenzo $[c d, l m]$ anthanthrene

Dibenzo $[c d, l m]$ perylene

Dibenzo $[c d, n]$ naphtho[3,2,1,8-pqra]perylene

Dibenzo[de, $i j]$ naphtho[3,2,1,8,7-rstuv]pentaphene

Dibenzo[de,ij]naphtho[7,8,1,2,3-pqrst $]$ pentaphene

Dibenzo[de, $i j]$ pentaphene

Dibenzo[de,kl]pentaphene

Dibenzo[de,mn]naphthacene

Dibenzo[de,mn]naphtho[2,1,8-qra]naphthacene

Dibenzo[de,op]naphthacene

Dibenzo[de,qr]naphthacene

Dibenzo[de,qr]pentacene

Dibenzo[de,qr]tetracene

Dibenzo[de,st]pentacene

Dibenzo[de, $u v]$ pentacene

Dibenzo $[d e, u v]$ pentaphene

Dibenzo[def,mno]chrysene

Dibenzo[def,mno]cyclopenta $[h i]$ chrysene

Dibenzo[def,p]chrysene

Dibenzo[e,ghi]perylene

Dibenzo[e,l]pyrene

Dibenzo $[e f, h i]$ naphtho $[8,1,2-a b c]$ coronene

Dibenzo[ef,no]naphtho[8,1,2-abc]coronene

Dibenzo $[f, j]$ naphtho[1,2,3,4-pqr $]$ picene

Dibenzo $[f, j]$ picene

Dibenzo $[f, m]$ tetraphene

Dibenzo $[f, p q r]$ picene

Dibenzo[f,s]picene

Dibenzo $[f g, i j]$ naphtho[2,1,8-uva]pentaphene

Dibenzo $[f g, i j]$ naphtho[7,8,1,2,3-pqrst $]$ pentaphene

Dibenzo $[f g, i j]$ pentaphene

Dibenzo $[f g, i j]$ phenanthro[2,1,10,9,8,7-

pqrstuv|pentaphene

Dibenzo $[f g, i j]$ phenanthro[9,10,1,2,3-pqrst $]$ pentaphene

Dibenzo $[f g, o p]$ anthanthrene

Dibenzo $[f g, o p]$ naphthacene

Dibenzo $[f g, q r]$ pentacene

Dibenzo $[f g, s t]$ hexacene

Dibenzo[fgh,pqr]trinaphthylene
Dibenzo[g,p]chrysene

Dibenzo $[$ ghi,lm $]$ naphtho[1,8-ab $]$ perylene $\quad 537$

Dibenzo[ghi,mno]fluoranthene $\quad 53$

Dibenzo[ghi,n]naphtho[8,1,2-bcd $]$ perylene $\quad 544$

Dibenzo[ghi,pqr]perylene $\quad 159$

Dibenzo $[\boldsymbol{h}, \boldsymbol{r s t}]$ pentaphene $\quad 336$

Dibenzo $[h \boldsymbol{h i}, \boldsymbol{k} l]$ naphtho[8,1,2-abc]coronene $\quad 624$

Dibenzo[hi,qr]anthanthrene 318

Dibenzo[ $[i j, r s t]$ naphtho[2,1,8,7-defg]pentaphene $\quad 554$

Dibenzo[ij,rst $]$ phenanthro[9,10,1,2-defg $]$ pentaphene $\quad 641$

Dibenzo[ijk,tuv]peropyrene 376

Dibenzo $[j, l]$ fluoranthene 213

Dibenzo[j,lm $]$ naphtho[1,8-ab]perylene $\quad 593$

Dibenzo $[j, l m]$ phenanthro $[5,4,3-a b c d]$ perylene $\quad 636$

Dibenzo $[k l, n o]$ naphtho $[8,1,2-a b c]$ coronene $\quad 621$

Dibenzo $[k l, r s t]$ naphtho[2,1,8,7-defg $]$ pentaphene $\quad 556$

Dibenzo[mn,qr]fluoreno[2,1,9,8,7-defghi]naphthacene 373

Dibenzo[pq,uv]pentaphene $\quad 346$

Dibenzo $[q, v w x]$ hexaphene $\quad 568$

Dibenzo $[\boldsymbol{r s}, v w x]$ naphtho[2,1,8,7-klmn $]$ hexaphene $\quad 638$

Dibenzo-1,2,7,8-anthracene $\quad 127$

Dibenzo-2,3,11,12-fluoranthene 225

1,2,7,8-Dibenzo-4,5-phenanthrylenemethane 150

1,2,5,6-Dibenzoanthracene 130

2,3,6,7-Dibenzoanthracene 133

2,3,4,5-Dibenzocoronene 374

2,3,8,9-Dibenzocoronene 376

anti-diperi-Dibenzocoronene 376

vic-diperi-Dibenzocoronene 374

2,3,5,6-Dibenzofluoranthene 214

1,2,3,4-Dibenzofluorene $\quad 84$

1,2,5,6-Dibenzofluorene 90

1,2,7,8-Dibenzofluorene 92

2,3,6,7-Dibenzofluorene 96

1.12,2.3-Dibenzoperylene 266

1.12,4.5-Dibenzoperylene 265

2,3,10,11-Dibenzoperylene $\quad 352$

2,3,8,9-Dibenzoperylene 356

1,2,5,6-Dibenzophenanthrene $\quad 126$

2,3,6,7-Dibenzophenanthrene 128

2,3:7,8-Dibenzophenanthrene 131

3,4,5,6-Dibenzophenanthrene $\quad 122$

$\beta, \beta^{\prime}$-Dibenzophenanthrene 128

$\gamma, \gamma^{\prime}$-Dibenzophenanthrene $\quad 122$

1,2:4,5-Dibenzopyrene 234

1,2:9,10-Dibenzopyrene 232

2,3:4,5-Dibenzopyrene 232

3,4:8,9-Dibenzopyrene 232

3,4:9,10-Dibenzopyrene 243

4,5,6,7-Dibenzopyrene 232

4,5,8,9-Dibenzopyrene 243

4,5,9,10-Dibenzopyrene $\quad 237$

1.2,7.8-Dibenzotetracene 300 
1.2,9.10-Dibenzotetracene

1.2,5.6-Dibenzotetraphene

1,2:7,8-Dibenzphenanthrene

1,2:3,4-Dibenzpyrene

1,2:6,7-Dibenzpyrene

1,2:7,8-Dibenzpyrene

3,4:8,9-Dibenzpyrene

Dicyclopenta $[a, c]$ naphthacene

Dicyclopenta $[a, j]$ coronene

Difluorenylene

1,2-Dihydroacenaphthylene

1,2-Dihydroben $[j]$ aceanthrylene

10,15-Dihydrotribenzo $[a, f, k]$ trindene

Diindeno[1,2,3-cd:1',d',3'-jk]pyrene

Diindeno[1,2,3-de, $\left.1^{\prime}, 2^{\prime}, 3^{\prime}-k l\right]$ anthracene

Dinaphth [1,2-a:1',2'-h] anthracene

Dinaphth[1,2-a:2',1'-j] anthracene

Dinaphth $\left[2,3-a, 2^{\prime}, 3^{\prime}-c\right]$ anthracene

peri-Dinaphthalene

lin-Dinaphthanthracene

Dinaphtho[1,2,3-cd, 1',2',3'-lm]perylene

Dinaphtho[1,2,3-cd,3',2',1'-lm]perylene

Dinaphtho[1,2,3-fg:1',2',3'-qr]pentacene

Dinaphtho $\left[1,2,3-f g: 3 ', 2^{\prime}, 1^{\prime}-q r\right]$ pentacene

Dinaphtho[1,2-b,2',1'-n]perylene

Dinaphtho[1,2-b:1',2'-k]chrysene

Dinaphtho[1,8-ab:8',1',2',3'-fghi]perylene

Dinaphtho[1,8-bc:1',8'-mn]picene

Dinaphtho[2,1,8,7-defg:2',1',8',7'-ijkl]pentaphene

Dinaphtho[2,1,8,7-defg:2',1',8',7'-opqr]pentacene

Dinaphtho[2,1,8,7-defg:2',1',8',7'-qrst $]$ pentacene

Dinaphtho[2,1,8-cde,2',1',8'-lmn]perylene

Dinaphtho[2,1,8-fgh:3',2',1',8',7'-rstuv]pentaphene

Dinaphtho[2,1,8-fgh:7',8',1',2',3'-pqrst]pentaphene

Dinaphtho[2,1,8-jkl:2',1',8'-uva]pentacene

Dinaphtho[2,1-a:1',2'-l]naphthacene

Dinaphtho[2,1-a:2',1'-j]naphthacene

Dinaphtho[2,1-c 1',2'-g]phenanthrene

Dinaphtho[2,3-c:2',3'-m]pentaphene

Dinaphtho[3,2,1-fg:1',2',3'-ij]pentaphene

Dinaphtho[3,2,1-fg:3',2',1'-qr]pentacene

Dinaphtho[8,1,2-abc:2',1',8'-efg]coronene

Dinaphtho[8,1,2-abc:2',1',8'-hij]coronene

Dinaphtho $\left[8,1,2-a b c: 2^{\prime}, 1^{\prime}, 8^{\prime}-k l m\right]$ coronene

Dinaphtho $\left[8,1,2-a b c: 2^{\prime}, 1^{\prime}, 8^{\prime}\right.$-nop $]$ coronene

Dinaphtho[8,1,2-abc:8',1',2'-ghi]coronene

Dinaphtho[8,1,2-abc:8',1',2'-jkl]coronene

Dinaphtho[8,1,2-cde:7',8',1',2',3'-pqrst $]$ pentaphene

Dinaphtho[8,1,2-Imn:2',1',8'-qra]naphthacene

$\alpha, \alpha^{\prime}$-Dinaphthofluorene

Diphenanthro[3,4-c:4',3'-g]phenanthrene

Diphenylenemethane

9,10-Diphenylenephenanthrene
2,3,3',2'-Dipyrenylene

1,8-Ethylenenaphthalene

Fluoranthene

18

Fluorantheno[8,9-b]triphenylene $\quad 563$

$9 H$-Fluorene 11

Fluorene

Fluoreno[2,1-a]fluorene

Fluoreno[2,3-a]fluorene

Fluoreno[3,2,1,9-defg]chrysene

Fluoreno[3,2-b]fluorene

Fluoreno[3,4-b]fluorene

Fluoreno[4,3,2-de]anthracene

Fluoreno[4,3-c]fluorene

Fluoreno[9,1-ab]triphenylene

[6] Helicene

Heptacene

Heptaphene

Hexabenzobenzene

Hexacene

Hexahelicene

Hexaphene

Idryl

as-Indacene

$s$-Indacene

as-Indaceno $[2,3-a]$ phenanthrene

$1 H$-Indene

Indene

Indeno[1,2,3-cd]fluoranthene

Indeno $[1,2,3-c d]$ perylene

Indeno[1,2,3-cd]pyrene

Indeno[1,2,3-de]naphthacene

Indeno[1,2,3- $f g]$ naphthacene

Indeno[1,2,3-hi]chrysene

$8 H$-Indeno[1,2-a]anthracene

Indeno[1,2- $a]$ phenalene

$7 H$-Indeno[1,2- $a]$ phenanthrene

$7 H$-Indeno[1,2- $a]$ pyrene

$11 H$-Indeno[1,2-a] triphenylene

$13 H$-Indeno[1,2-b]anthracene

$12 H$-Indeno[1,2-b]phenanthrene

13H-Indeno[1,2-c]phenanthrene

9H-Indeno[1,2-e]pyrene

13H-Indeno[1,2-l]phenanthrene

Indeno[1,7,6,5-cdef]chrysene

Indeno[1,7a-a]phenanthrene

Indeno $[1,7-a b]$ chrysene

Indeno[1,7-ab]pyrene

Indeno $[1,7-a b]$ triphenylene

$11 \mathrm{H}$-Indeno[2,1,7-cde]pyrene

$3 H$-Indeno[2,1,7-cde]pyrene

13H-Indeno[2,1,7-qra]naphthacene

13H-Indeno[2,1- $a$ ] anthracene

$5 H$-Indeno[2,1- $a]$ chrysene
11

171

169

258

173

167

177

168

321

271

521

497

159

$\mathbf{3 0 7}$

271

298 
Indeno[2,1- $a]$ phenalene

$11 H$-Indeno $[2,1-a]$ phenanthrene

$11 H$-Indeno[2,1-a]pyrene

$8 H$-Indeno[2,1-b]phenanthrene

9H-Indeno[2,1-c]phenanthrene

Indeno[3,2,1,7-defg]chrysene

Indeno $[4,3,2,1-c d e f]$ chrysene

Indeno[5,6,7,1-defg]chrysene

Indeno[5,6,7,1-pqra]perylene

Indeno[6,7,1,2-defg $]$ naphthacene

$1 H$-Indeno[6,7,1-mna] anthracene

Indeno[7,1,2,3-cdef $]$ chrysene

4H-Indeno[7,1,2-ghi]chrysene

Indeno[7,1- $a b]$ naphthacene

Indeno[7,1-ab]triphenylene

Indeno[7,1-bc]chrysene

Indeno-2',3'-3,4-pyrene

Isochrysene

Isochrysofluorene

Isonaphthofluorene

1',9-Methylene-1,2,5,6-dibenzanthracene

1',9-Methylene-1,2-benzanthracene

2,2'-Methylenebiphenyl

4,5-Methylenephenanthrene

Naphth $\left[1^{\prime}, 2^{\prime}: 5,6\right]$ indeno[1,2,3-cd] pyrene

Naphth $[1,2-a]$ aceanthrylene

Naphth [1,2-a]acephenanthrylene

Naphth[1,2- $a]$ anthracene

Naphth[1,2- $d]$ acenaphthylene

Naphth $[1,2-e]$ acephenanthrylene

Naphth[1,2-j] aceanthrylene

Naphth[1,2-k] acephenanthrylene

Naphth $\left[2^{\prime}, 1^{\prime}: 4,5\right]$ indeno[1,2,3-cd]pyrene

Naphth[2,1-a] aceanthrylene

Naphth[2,1-a]anthracene

Naphth[2,1-d]acenaphthylene

Naphth[2,1-e]aceanthrylene

Naphth[2,1-e] acephenanthrylene

Naphth[2,1-k] acephenanthrylene

Naphth[2,1-l]aceanthrylene

Naphth[2,1-l]acephenanthrylene

Naphth[2,3-a] aceanthrylene

Naphth[2,3-e]acenaphthylene

Naphth[2,3-e] acephenanthrylene

Naphth[2,3-l] acephenanthrylene

$5 H$-Naphth $[3,2,1-d e]$ anthrene

1,9,8-(diperi)-Naphth-2,9-dihydroanthracene

2',1'-Naphtha-1,2-fluorene

1',2'-Naphtha-2,3-fluorene

1',3'-Naphtha-3,4-pyrene

Naphthacene

Naphthaceno[2,1,12,11-opqra]naphthacene

Naphthaceno[4,5,6,7,8-defghij]naphthacene
peri-Naphthacenonaphthacene

1,2-(1,8-Naphthalenediyl)benzene

Naphthalin

Naphthanthracene

Naphthanthracene

1,8,9-Naphthanthrene

$13 H$-meso- $\alpha$-Naphthanthrene

$8 H$-meso- $\alpha$-Naphthanthrene

lin-Naphthanthrene

Naphthanthrene

$1 H$ - $\alpha$-Naphthindene

$1 H$ - $\beta$-Naphthindene

$3 H$ - $\alpha$-Naphthindene

Naphtho(2',3':7,8)fluoranthene

Naphtho(2',3':8,9)fluoranthene

Naphtho-(2'3':4,5)pyrene

Naphtho[1,2,3,4-def $]$ chrysene

Naphtho[1,2,3,4-ghi]fluoranthene

Naphtho[1,2,3,4-ghi]perylene

Naphtho[1,2,3,4-rst]pentaphene

9H-Naphtho[1,2,3-cd]perylene

6H-Naphtho[1,2,3-cd]pyrene

Naphtho[1,2-a]coronene

Naphtho[1,2-a]fluoranthene

Naphtho[1,2-a]naphthacene

Naphtho[1,2-a]pentacene

Naphtho[1,2-a]pentaphene

Naphtho[1,2-a]pyrene

Naphtho[1,2-a]tetracene

Naphtho[1,2-a]tetraphene

Naphtho[1,2-b]chrysene

Naphtho[1,2-b]fluoranthene

$12 H$-Naphtho[1,2-b]fluorene

Naphtho[1,2-b]perylene

Naphtho[1,2-b]picene

Naphtho[1,2-b]triphenylene

Naphtho[1,2-c]chrysene

Naphtho[1,2-c]pentaphene

Naphtho[1,2-e]pyrene

Naphtho[1,2-f]picene

Naphtho[1,2-g]chrysene

Naphtho[1,2- $h]$ pentaphene

Naphtho[1,2-j]fluoranthene

Naphtho[1,2-k]fluoranthene

Naphtho[1,8,7,6-cdef]fluorene

Naphtho[2,1,8-def]picene

Naphtho[2,1,8-fgh]pentaphene

Naphtho[2,1,8-hij] anthanthrene

Naphtho[2,1,8-qra]naphthacene

Naphtho[2,1,8-uva]pentacene

Naphtho[2,1,8-uva]pentaphene

Naphtho[2,1,8-yza]hexacene 
Naphtho[2,1-a]fluoranthene

$11 H$-Naphtho[2,1- $a$ ] fluorene

Naphtho[2,1-a]naphthacene

Naphtho[2,1-a]pentaphene

Naphtho[2,1-a]picene

Naphtho[2,1-a]pyrene

Naphtho[2,1-a]tetraphene

Naphtho[2,1-b]chrysene

Naphtho[2,1-b]fluoranthene

Naphtho[2,1-b]perylene

Naphtho[2,1-b]picene

Naphtho[2,1-c:7,8-c']diphenanthrene

Naphtho[2,1-c]chrysene

Naphtho[2,1-c]pentaphene

Naphtho[2,1-c]picene

Naphtho[2,1-c]tetraphene

Naphtho[2,1-j]fluoranthene

Naphtho[2',3':2,3]fluoranthene

Naphtho[2,3- $a]$ coronene

Naphtho[2,3-a]fluoranthene

13H-Naphtho[2,3- $a$ ]fluorene

Naphtho[2,3-a]pentaphene

Naphtho[2,3-a]picene

Naphtho[2,3-a]pyrene

Naphtho[2,3- $a$ ]tetraphene

Naphtho[2,3-b]fluoranthene

Naphtho[2,3-b]picene

Naphtho[2,3-b]pyrene

Naphtho[2,3-c]chrysene

$8 H$-Naphtho[2,3-c]fluorene

Naphtho[2,3-c]pentaphene

Naphtho[2,3-e]pyrene

Naphtho[2,3-g]chrysene

Naphtho[2,3-h]pentaphene

Naphtho[2,3-j]fluoranthene

Naphtho[2,3-k]fluoranthene

Naphtho[2,3-s]picene

Naphtho[2'.1',1.2]tetracene

Naphtho[2'.3',1.2]pyrene

Naphtho[3,2,1,8,7-defgh]pyranthrene

Naphtho[3,2,1,8,7-vwxyz]hexaphene

Naphtho[3,2,1-jk]fluorene

Naphtho[3',4':3,4]pyrene

Naphtho[4,5,6-abc]aceanthrylene

Naphtho[5,4,3-abc]coronene

Naphtho[7,8,1,2,3-pqrst]pentaphene

Naphtho[7,8,1,2,3-tuvwx] $]$ exaphene

Naphtho[8,1,2-abc]coronene

Naphtho[8,1,2-bcd] perylene

Naphtho[8,1,2-cde]naphthacene

Naphtho[8,1,2-cde]pentaphene

Naphtho[8,1,2-efg]anthanthrene

Naphtho[8,1,2-ghi]chrysene
Naphtho $\left[b^{\prime}, b\right]$ chrysene

1',2'-Naphtho-1,2-fluoranthene 215

Naphtho-2',3',1,2-anthracene 128

Naphtho-2',3',1,2-phenanthrene 131

Naphtho-2',3',2,3-phenanthrene 129

Naphtho-2',3',3,4-phenanthrene $\quad 125$

Naphthobenzanthrene 106

2,3- $\beta$-Naphthofluorene $\quad 98$

lin-Naphthofluorene $\quad 98$

Nonacene $\quad 660$

Octacene $\quad 612$

Ovalene $\quad 522$

Paranaphthalene 15

Pentacene 133

peri-Pentacenopentacene $\quad 587$

Pentalene 2

Pentaleno[1,2-b:4,5- $\left.b^{\prime}\right]$ dinaphthalene 172

Pentanthrene 9

Pentanthrene 10

Pentaphene $\quad 128$

Perinaphthene 13

Peropyrene 270

Perylene 73

Perylo[3,2,1,12-pqrab]perylene $\quad 581$

$1 H$-Phenalene 13

Phenalene $\quad 13$

Phenaleno[12,3,4-ghij]perylene $\quad 314$

Phenalin 13

2',3'-Phenanthra-1,2-anthracene 299

2',3'-Phenanthra-2,3-phenanthrene 297

Phenanthrene $\quad 14$

[Phenanthreno-9',10':9,10]phenanthrene-1,1'methylene $\quad 308$

Phenanthrin 14

Phenanthrindene $\quad 22$

Phenanthro[1,10,9,8-opqra $]$ perylene $\quad 313$

Phenanthro[1,2,3,4-def $]$ chrysene $\quad 325$

Phenanthro[1,2,3,4-ghi]perylene 392

Phenanthro[1,2-a]naphthacene $\quad 472$

Phenanthro[1,2-b]chrysene $\quad 519$

Phenanthro[1,2-b]triphenylene 473

Phenanthro[10,1,2,3-cdef $\mid$ fluorene $\quad 115$

Phenanthro[10,1,2-abc]coronene $\quad 583$

Phenanthro[2,1,10,9,8,7-pqrstuv]pentaphene 319

Phenanthro[2,1,10,9,8,7-tuvwxyz]hexaphene 561

Phenanthro[2,1-b]chrysene $\quad 503$

Phenanthro[2,1-f]picene $\quad 603$

Phenanthro[2,3,4,5-tuvab]picene $\quad 420$

Phenanthro[2,3-c]chrysene $\quad 450$

Phenanthro[2,3-g]chrysene 445

Phenanthro[3,2-b]chrysene $\quad 514$

Phenanthro $[3,2-g]$ chrysene $\quad 436$

Phenanthro[3,4-a]anthracene 296

Phenanthro[3,4-a]naphthacene $\quad 504$ 
Phenanthro $[3,4-b]$ chrysene

Phenanthro $[3,4-b]$ triphenylene

Phenanthro[3,4-c]chrysene

Phenanthro[3,4-c]phenanthrene

Phenanthro[4,3,2,1-def]chrysene

Phenanthro[4,3- $a]$ anthracene

Phenanthro[4,3-b]chrysene

Phenanthro[5,4,3,2-abcde]perylene

Phenanthro[9,10,1,2,3-pqrst $]$ pentaphene

Phenanthro[9,10,1-qra]naphthacene

Phenanthro $[9,10-a]$ naphthacene

Phenanthro[9,10-b]chrysene

Phenanthro[9,10-b]triphenylene

4,5-Phenanthrylenemethane

Phene

5,6-(1,2-Phenylene)naphthacene

1,10-(1,2-Phenylene)pyrene

1,10-(o-Phenylene)pyrene

2,3-(o-Phenylene)pyrene

1,9-Phenyleneanthracene

10,11-Phenylenenaphthacene

5,6-o-Phenylenenaphthacene

2,3-Phenylenepyrene

o-Phenylenepyrene

Picene

Pyranthrene

Pyren

Pyrene

peri-Pyrene-1,10(CH2)-indene

Pyrenindene

Pyreno[1,10,9-abc]coronene

Pyreno[10,1,2-abc]coronene

Pyreno[2,1-b]picene

Pyreno[5,4,3,2,1-pqrst $]$ pentaphene

Rubicene

Terrylene

Tetrabenz $[a, c, h, j]$ anthracene

Tetrabenzo $[a, c, h i, m n]$ naphthacene

Tetrabenzo $[a, c, h i, q r]$ pentacene

Tetrabenzo $[a, c, j, l]$ naphthacene

Tetrabenzo[a,c,l,n]pentacene

Tetrabenzo $[a, c d, f, l m]$ perylene

Tetrabenzo $[a, c d, j, l m]$ perylene

Tetrabenzo $[a, e, j, o]$ perylene
Tetrabenzo $[a, f, j, 0]$ perylene

646

Tetrabenzo $[a, f, k, n]$ perylene

Tetrabenzo[bc,ef,hi,kl]coronene

618

Tetrabenzo $[b c, e f, k l, n o]$ coronene $\quad 616$

Tetrabenzo[de,h,kl,rst]$]$ pentaphene $\quad 594$

Tetrabenzo[de,hi,mn,qr]naphthacene $\quad 387$

Tetrabenzo[de,hi,op,st]pentacene $\quad 596$

Tetrabenzo[de,jk,op,uv]pentacene $\quad \mathbf{5 9 5}$

1,2:3,4:5,6:7,8-Tetrabenzonaphthalene 272

Tetracene

Tetrahelicene

Tetraphene

Tribenz $[a, c, h]$ anthracene

1,2,3,4,5,6-Tribenzanthracene

Tribenzo $[a, c, j]$ naphthacene

$8 H$-Tribenzo[ $[a, c d, l]$ pyrene

Tribenzo[a,cd,lm]perylene

Tribenzo[a,e,ghi]perylene

Tribenzo[a,ef,hi]coronene

Tribenzo[a,ef,no]coronene

Tribenzo $[a, f, j]$ perylene

Tribenzo[a,ghi,k]perylene

Tribenzo[a,hi,kl]coronene

Tribenzo[a,hi,mn]naphthacene

Tribenzo[a,i,l]pyrene

Tribenzo[b,def,p]chrysene

Tribenzo[b,e,ghi]perylene

Tribenzo $[b, g, k]$ chrysene

Tribenzo $[b, g, l]$ chrysene

Tribenzo $[b, g, p]$ chrysene

Tribenzo[b,n,pqr]perylene

Tribenzo[c,g,mno]chrysene

Tribenzo[de,ij,rst $]$ pentaphene

Tribenzo[de,kl,rst]pentaphene

Tribenzo[fgh,pqr,zalb1] trinaphthylene

Tribenzo[jk,qr,uv]naphtho[2,1,8,7-defg]pentacene

Tribenzobicyclo[2.2.2]octatriene

Triindeno[2,3:2',3':2",3"]benzene

Trinaphthylene

Trinaphthylenebenzene

Triphenylene

Triptycene

Truxene

Zethrene 
1<smiles>[C]1CCCCC1</smiles>

Benzene

[6]Annulene

Benzol

Benzine

Cyclohexatriene

Phene

Pentalene

Indene

$1 \mathrm{H}$-Indene

Naphthalene

Naphthalin

as-Indacene

s-Indacene

Acenaphthylene

Acenaphthalene

Cyclopenta[de]naphthalene

1,2-dihydroacenaphthylene

1,8-ethylenenaphthalene

$3 H$-Benz[e]indene

$3 \mathrm{H}$-a-Naphthindene

Pentanthrene

10<smiles>C1CC2CCC3CCCC3C2C1</smiles>

$1 H$-Benz[e]indene

$1 \mathrm{H}$-a-Naphthindene Pentanthrene
208-96-8 $\quad \mathrm{C}_{12} \mathrm{H}_{8}$

83-32-9

$\mathrm{C}_{13} \mathrm{H}_{10}$

166

1.362

10.91

4.234

$71-43-2$

250-25-9

$95-13-6$

$\mathrm{C}_{9} \mathrm{H}_{8}$

116

1.248

9.148

7.332
4.235

91-20-3 $\quad \mathrm{C}_{10} \mathrm{H}_{8}$

128

1.238

9.195

7.428

210-65-1 $\quad \mathrm{C}_{12} \mathrm{H}_{8}$

152

1.308

10.32
7.888
3.887

3.887

1.494

7.526

3.884

1.072

9.242

8.624
3.882

232-54-2

$\mathrm{C}_{13} \mathrm{H}_{10}$

166

1.368

10.97

8.023 
11<smiles>C1=CC2CC3CCCCC3C2C1</smiles>

12<smiles>C1=CC2CC3CCCCC3CC2C1</smiles>

13<smiles>C1=CC2CCCC3CCCC(C1)C23</smiles>

14<smiles>C1CCC2CCCCC2C1</smiles>

15<smiles>C1CCC2CC3CCCCC3CC2C1</smiles>

16<smiles>C1=CC2CCC3CCCC4CCC(C1)C2C34</smiles>

17<smiles>C1=CC2CCCC3CCC4CCCC(C1)C4C23</smiles>

18<smiles>C1CC2CCCC3C(C1)CCCC23</smiles>

Fluorene

$9 \mathrm{H}$-Fluorene o-Biphenylenemethane

Diphenylenemethane

2,2'-Methylenebiphenyl

o-Biphenylmethane

$1 H$-Benz[f]indene

$1 \mathrm{H}$-b-Naphthindene

Phenalene

$1 \mathrm{H}$-Benzonaphthene

Benzonaphthene

Perinaphthene

$1 \mathrm{H}$-Phenalene

Phenalin

Phenanthrene

Phenanthrin

Anthracene

Anthracin
Paranaphthalene

$5 H$-Benz[ $f g]$ acenaphthylene

4H-Cyclopenta[def]phenanthrene

Benzo[def]fluorene

Cyclopentaphenanthrene

4,5-Methylenephenanthrene

4,5-Phenanthrylenemethane

Fluoranthene

Benzo[jk]fluorene

Idryl

1,2-(1,8-Naphthalenediyl)benzene

Benz[a]acenaphthylene

1,2-Benzoacenaphthylene
86-73-7

$\mathrm{C}_{13} \mathrm{H}_{10}$

166

1.520

11.43

7.521
4.241

$268-40-6$

$\mathrm{C}_{13} \mathrm{H}_{10}$

166

1.541

11.49

7.455
4.236

203-80-5

$\mathrm{C}_{13} \mathrm{H}_{10}$

166

1.052

9.640

9.165

4.205

85-01-8

$\mathrm{C}_{14} \mathrm{H}_{10}$

178

1.463

11.75

8.031

3.888

120-12-7

$\mathrm{C}_{14} \mathrm{H}_{10}$

178

1.566

11.65

7.439
3.882

3.882

$\mathrm{C}_{15} \mathrm{H}_{10}$

190

1.181

10.88

9.211
4.204

203-64-5 $\quad \mathrm{C}_{15} \mathrm{H}_{10} \quad 190 \quad 1.288$

11.31
8777

4.241

206-44-0

$\mathrm{C}_{16} \mathrm{H}_{10}$

202

1.208

11.16

9.240
3.884 
19<smiles>C1=CC2CC3CCCC4CC(C1)CCCC243</smiles>

20<smiles>C1=CC2CCC3CC4CCCCC4C3CCC2C1</smiles>

21<smiles>C1CC2CCC3CCCC4CCC(C1)C2C34</smiles>

22<smiles>C1=CC2C(CC1)C1CCCCC1C1CCCC21</smiles>

23<smiles>C1=CC2C(CC1)C1CCCC1C1CCCCC21</smiles>

24<smiles>C1CC2CC3CCC4CCCCC4C3CC2C1</smiles>

25<smiles></smiles>

26<smiles>C1=CC2C(C1)CCC1C3CCCCC3CCC21</smiles>

27<smiles>C1=CC2C3=C(CCC4CCCCC34)C2C1</smiles>

numbering exception

numbering exception
Acephenanthrylene

Cyclopenta[jk]phenanthrene

Aceanthrylene

Cyclopenta[de]anthracene

Pyrene

Benzo[def]phenanthrene Pyren

$1 \mathrm{H}$-Cyclopenta[/]phenanthrene Phenanthrindene

$2 \mathrm{H}$-Cyclopenta[I]phenanthrene 3,4-(o,o'-Biphenylene)cyclopentadiene

$8 H$-Cyclopenta[b]phenanthrene

1H-Cyclopent[a]anthracene

17H-Cyclopenta[a]phenanthrene 1,2-Cyclo- $\Delta^{1,4}$-pentadienophenanthrene

$15 \mathrm{H}$-Cyclopenta[a]phenanthrene 1,2-Cyclo- $\Delta^{1,3}$-pentadienophenanthrene

$227-50-9$
$129-00-0$

201-06-9

$\mathrm{C}_{16} \mathrm{H}_{10}$

202

1.291

11.70

9.067

202-03-9

$\mathrm{C}_{16} \mathrm{H}_{10}$

202

1.354

11.70

3.883

$\mathrm{C}_{17} \mathrm{H}_{12}$

216

1.139

11.76

10.33

11.66

3.888

$\mathrm{C}_{17} \mathrm{H}_{12}$

216

1.173

11.76

10.03
4.244

$\mathrm{C}_{17} \mathrm{H}_{12}$

216

1.581

13.19

8.346

$\mathrm{C}_{17} \mathrm{H}_{12}$

216

1.625

13.05

8.032

$\mathrm{C}_{17} \mathrm{H}_{12} \quad 216 \quad 1.632$

13.12 8.037 
28<smiles>C1CCC2CC3CC4CCCC4CC3CC2C1</smiles>

$1 H$-Cyclopent $[b]$ anthracene

$7 H$-Benzo[c]fluorene

Benzo[c]fluorene

3,4-Benzofluorene

$\mathrm{H}_{2}$

30<smiles>C1=CC2CCC3C4CCCCC4CC3C2CC1</smiles>

31<smiles>C1CCC2CC3CC4CCCCC4CC3CC2C1</smiles>

32<smiles>C1=CC2CCCC3CC4CCCCC4CC3CCC2C1</smiles>

33<smiles>C1=CC2CC3CCC4CCCC5CCC(C1)C2C3C54</smiles>

34<smiles>C1CC2CCC3CC4CCCC5CC(C1)C2C3C54</smiles>

35<smiles>C1CC2CCC3CCC4CCCC5CCC(C1)C2C5C43</smiles>

$11 \mathrm{H}$-Benzo[a]fluorene

Benzo[a]fluorene

1,2-Benzofluorene

$11 \mathrm{H}$-Benzo[b]fluorene

2,3-Benzofluorene Isonaphthofluorene

$1 H$-Benz[de]anthracene

Benzanthrene

1H-meso-Benzanthrene

Cyclopenta[cd]pyrene
Acepyrene Acepyrylene

Benz[mno]aceanthrylene

Naphtho[1,8,7,6-cdeffluorene

Benzo[ghifluoranthene

Benzo[mno]fluoranthene

2,13-Benzofluoranthene

7,10-Benzofluoranthene

Benzofluoranthene Chrysofluorene
259-06-3 $\quad \mathrm{C}_{17} \mathrm{H}_{12} \quad 216 \quad \begin{aligned} & 1.866 \\ & 13.94\end{aligned}$

13.94
7.472

4.237

$205-12-9$

$\mathrm{C}_{17} \mathrm{H}_{12}$

216

1.384

12.65

9.138

238-84-6 $\quad \mathrm{C}_{17} \mathrm{H}_{12}$

216

1.669

13.39

4.242

243-17-4

$\mathrm{C}_{17} \mathrm{H}_{12}$

216

1.768

13.76

7.783

199-95-1 $\quad \mathrm{C}_{17} \mathrm{H}_{12}$

216

1.274

11.77

9.238

4.217

27208-37-3 $\quad \mathrm{C}_{18} \mathrm{H}_{10} \quad 226 \quad 1.200$

11.52

9.602

3.887

203-13-4 $\quad \mathrm{C}_{18} \mathrm{H}_{10} \quad 226 \quad 1.222$

11.83

9.679

3.884

203-12-3 $\quad \mathrm{C}_{18} \mathrm{H}_{10} \quad 226 \quad 1.179$

11.40

9.664 
36<smiles>C1CCC2C(C1)C1CCCCC1C1CCCCC21</smiles>

37<smiles>C1=CC2C(CC1)CC1CCC3CCCCC3C12</smiles>

38<smiles>C1CCC2CC3C(CCC4CCCCC43)CC2C1</smiles>

39<smiles>C1CCC2C(C1)CCC1CCCCC12</smiles>

40<smiles>C1CCC2CC3CC4CCCCC4CC3CC2CC1</smiles>

41<smiles>C1CC2C(C1)C1CCCC3CCC4CCCC2C4C31</smiles>

42<smiles>C1=CC2C(CC1)CC1CCC3CCCC2C1C3</smiles>

$1 H$-Cyclopenta[a]pyrene

$1 H$-Benz[fg]aceanthrylene

Benzo[I]phenanthrene Isochrysene 1,2,3,4-Dibenznaphthalene

Benzo[c]phenanthrene

3,4-Benzophenanthrene Tetrahelicene
Benz[a]anthracene
Benzo $[b]$ phenanthrene 1,2-Benzanthracene 2,3-Benzophenanthrene 1,2-Benzanthrene
Naphthanthracene

Chrysene

Benzo[a]phenanthrene

1,2-Benzophenanthrene

Naphthacene

Benz $[b]$ anthracene

Tetracene

2,3-Benzanthracene

$1 H$-Cyclopenta[e]pyrene<smiles>C1=CC2CCCCC2C2CCCC3C1CCCC32</smiles><smiles></smiles>

217-59-4

$$
\mathrm{C}_{18} \mathrm{H}_{12}
$$

1.119

195-19-7

$\mathrm{C}_{18} \mathrm{H}_{12}$

228

1.277

11.91

9.323

10.44

4.373

218-01-9

$\mathrm{C}_{18} \mathrm{H}_{12}$

228

1.734

13.94

8.039

92-24-0

$\mathrm{C}_{18} \mathrm{H}_{12}$

228

1.896

14.12

7.446

109587-09-9 $\quad \mathrm{C}_{19} \mathrm{H}_{12}$

240

42315-22-0

$\mathrm{C}_{19} \mathrm{H}_{12}$

240

1.411

13.10

9.289

193-69-1

$\mathrm{C}_{19} \mathrm{H}_{12}$

240

1.154

11.81

10.24

4.242
$1 H$-Indeno[6,7,1-mna]anthracene

$1 H$-Benzo[cd]fluoranthene

44

$\mathrm{C}_{19} \mathrm{H}_{12}$

240

1.393

12.90 9.250
4.240

45<smiles>C1CCC2C3CCC4CCCC5CCC(C1)C2C5C43</smiles>

42126-84-1 $\quad \mathrm{C}_{19} \mathrm{H}_{12}$

240 
46<smiles></smiles>

4H-Cyclopenta[def]triphenylene

$11 \mathrm{H}$-Benz[bc]aceanthrylene

1',9-Methylene-1,2-benzanthracene

4H-Cyclopenta[def]chrysene

$3 \mathrm{H}$-Benzo[cd]pyrene

1,10-(peri)-Benzo-1,5-dihydropyrene

1,9,8-(diperi)-Naphth-2,9-dihydroanthracene peri-Pyrene-1,10(CH2)-indene<smiles>C1=CC2CCC3CC4CCCC5CCC(C1)C2C3C54</smiles>

50<smiles>C1=CC2CC3CCC4C5CCCCC5CC(C1)C4C23</smiles>

49<smiles>C1=CC2CCC3CCC4CCCC5CCC(C1)C2C5C43</smiles>

51<smiles>C1=CC2CCC3CCC4CCCC5CC(C1)C2C3C45</smiles>

52<smiles>C1=CC2CCC3CCC4CCCC5CCC(C1)C2C3C54</smiles>

53<smiles>C1CC2CCC3CCC4CCC5CCC(C1)C2C5C34</smiles>

54<smiles>C1CCC2C(C1)CC1C3CCCC4CC5CCC(CC1C23)C45</smiles>

Benzo[de]cyclopent[a]anthracene

2H-Benzo[cd]pyrene

Naphthanthrene

$6 \mathrm{H}$-Benzo[cd]pyrene

1,8,9-Naphthanthrene

$5 H$-Benzo[cd]pyrene

Pyrenindene

Dibenzo[ghi,mno]fluoranthene

Corannulene

$\begin{array}{llll}\text { 23992-32-7 } \quad \mathrm{C}_{19} \mathrm{H}_{12} \quad 240 & 1.116 \\ & & & 11.76 \\ & & 10.54 \\ & & .241\end{array}$

202-94-8

$\mathrm{C}_{19} \mathrm{H}_{12}$

240

1.477

13.37

9.053

4.242

202-98-2 $\quad \mathrm{C}_{19} \mathrm{H}_{12}$

240

1.552

13.62

4.241

191-32-2

$\mathrm{C}_{19} \mathrm{H}_{12}$

240

1.222

11.69

9.564
4.207

191-33-3

$\mathrm{C}_{19} \mathrm{H}_{12}$

240

1.232

11.741

9.527

191-34-4 $\quad \mathrm{C}_{19} \mathrm{H}_{12}$

240

1.233

11.92

9.674

4.207

5821-51-2 $\quad \mathrm{C}_{20} \mathrm{H}_{10}$

250

1.040

11.25

10.82

3.894

198-46-9 $\quad \mathrm{C}_{20} \mathrm{H}_{12}$

252

1.257

13.01

10.35
4.242

4.242

Benzo[fg]cyclopent[a]anthracene

200-63-5 $\quad \mathrm{C}_{20} \mathrm{H}_{12}$<smiles>C1CC2CCCC3CC(CCC2)C2CC4C(C1)CCC4CCC32</smiles> 
56<smiles>C1CCC2C(C1)CC1CC3CCCC3C3CCCCC3C12</smiles>

Benzo[de]cyclopent $[b]$ anthracene

Indeno[2,1-a]phenalene

Indeno[1,2-a]phenalene

Benz[a]acephenanthrylene

60<smiles>C1CC2CCCC3CCC4CC5CCC(C1)C2C5C34</smiles>

61<smiles>C1=CC2CC3C4CCCCC4CC4CCC(C2C1)C43</smiles>

62<smiles>C1CC2CCC3CCCC4CCC5CCC(C1)C2C4C35</smiles>

63<smiles>C1CC2CCC3C4CCCC3C(CC4)C3C(C1)CCCC23</smiles>

64<smiles>C1=CC2CCC3C4CC1C(CCC1CCCCC1C4)CCC23</smiles>

65<smiles>C1CCC2CC3CC4CCC5CCCC3C2CC(CC1)C54</smiles>

Benz[e]aceanthrylene

Naphth[1,2-d]acenaphthylene

Cyclopenta[hi]chrysene

Benz[]]acephenanthrylene

Benz[]]aceanthrylene

Naphth $[2,1-d]$ acenaphthylene

Cholanthrylene

Benz[k]acephenanthrylene Naphth[2,3-e]acenaphthylene
Benz[]acephenanthrylene

27706-08-7

$\mathrm{C}_{20} \mathrm{H}_{12}$

252

1.458

14.04

3.889

$\mathrm{C}_{20} \mathrm{H}_{12}$

252

1.422

13.61

9.573
4.131

198-19-6

$\mathrm{C}_{20} \mathrm{H}_{12}$

252

1.459

13.46
9.227

3.897

192-28-9 $\quad \mathrm{C}_{20} \mathrm{H}_{12} \quad 252 \quad 1.168$

12.28

10.52
4.567

$95690-49-6$

$\mathrm{C}_{20} \mathrm{H}_{12}$

252

1.395

12.99

9.309

4.731

199-54-2

$\mathrm{C}_{20} \mathrm{H}_{12}$

252

1.429

13.97
9.777

3.886

211-91-6

$\mathrm{C}_{20} \mathrm{H}_{12}$

252

1.479

13.57

9.171
4.534

216-48-8

$\mathrm{C}_{20} \mathrm{H}_{12}$

252

1.519

13.92

9.163
3.893

202-33-5

$\mathrm{C}_{20} \mathrm{H}_{12}$

252

1.523

13.88

9.113
212-41-9

$\mathrm{C}_{20} \mathrm{H}_{12}$ 
66<smiles>C1=CC2CCC3C4CC1C(CCC1CC5CCCCC5CC1C4)CC23</smiles>

Cyclopenta[de]naphthacene

Benz[d]aceanthrylene

Cyclopenta[fg]naphthacene

Benz[a]aceanthrylene

Benzo[a]fluoranthene

Dibenzo[c,Im]fluorene

Naphtho[3,2,1-jk]fluorene

1,9-Phenyleneanthracene

69<smiles>C1=CC2C3CCCC4C5CCCCC5C5CCCC(C1)C5C3C24</smiles>

70<smiles></smiles>

71

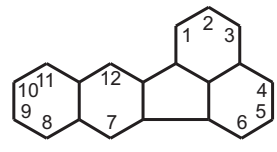

72<smiles>C1CC2CCCC3C4CCCC5C4CCCC(C1)C5C23</smiles>

73<smiles></smiles>

74<smiles>C1CC2CCC3CC4CCCC5CC(C1)C2C3C54</smiles>

7,8-Benzfluoranthene

Benzo[e]pyrene

Perylene

Dibenz [de, $k$ ] anthracene

Peri-dinaphthalene
Benz[e]acephenanthrylene

Benzo[b]fluoranthene

Benzo[e]fluoranthene

3,4-Benz[e]acephenanthrylene

2,3-Benzofluoranthene

3,4-Benzofluoranthene

Benzo[]fluoranthene

Benzo[/]fluoranthene

Dibenzo[a,jk]fluorene

10,11-Benzofluoranthene

\section{Benzo[k]fluoranthene \\ Dibenzo[ $b, j k]$ fluorene \\ 8,9-Benzofluoranthene \\ 11,12-Benzofluoranthene \\ 2,3:1',8'-Binaphthylene}

205-82-3

$\mathrm{C}_{20} \mathrm{H}_{12}$

252

1.399

13.41

9.583

207-08-9 $\quad \mathrm{C}_{20} \mathrm{H}_{12}$

252

1.474

13.62

9.2342

3.887

192-97-2

$\mathrm{C}_{20} \mathrm{H}_{12}$

252

1.118

11.76

10.52
3.887

198-55-0

$\mathrm{C}_{20} \mathrm{H}_{12}$

252

1.276

11.80

9.247
3.913

$$
\begin{gathered}
\text { Benzo[a]pyrene } \\
\text { Benzo[deffchrysene } \\
\text { 1,2-Benzpyrene } \\
\text { 3,4-Benzopyrene } \\
\text { 4,5-Benzpyrene } \\
\text { 6,7-Benzopyrene }
\end{gathered}
$$

50-32-8

$\mathrm{C}_{20} \mathrm{H}_{12}$

252

1.493

13.88

9.297
3.891 
75<smiles>C1=CC2CCC3CC4C5CCCCC5CCC4C(C1)C23</smiles>

76<smiles>C1CCC2C(C1)C1C3CCCCC3C3C2C2CCCCC2C13</smiles>

77<smiles>C1CC2CCC3CCC4CCC5CCC(C1)C1C2C3C4C51</smiles>

78<smiles>C1=CC2CCC3CC4CCCC5CCCC(C1)C2C5C43</smiles>

79

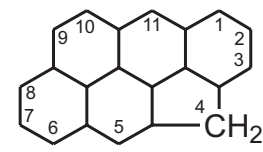

80<smiles>C1CCC2C(C1)C1CCCCC1C1C3CCCC3CCC21</smiles>

81<smiles>C1=CC2C(CC1)CCC1CC3C(CCC4CCCCC43)CC12</smiles>

82<smiles>C1=CC2CCC3CC4CC5CCCC5CC4CC3C2CC1</smiles>

9H-Benzo[a]cyclopent[]]anthracene

9,10-Dihydro-9,10[1',2']benzenoanthracene

Triptycene

Tribenzobicyclo[2.2.2]octatriene

$11 H$-Indeno[2,1,7-cde]pyrene

$3 H$-Indeno[2,1,7-cde]pyrene

$11 H$-Cyclopenta[ghi]perylene

4H-Benzo[def]cyclopenta[mno]chrysene

$11 \mathrm{H}$-Cyclopenta[a]triphenylene

$1 H$-Benzo[a]cyclopent[ $h]$ anthracene

9H-Indeno[2,1-c]phenanthrene
479-23-2

$\mathrm{C}_{20} \mathrm{H}_{14}$

254

1.568

13.93

8.881

4.222

477-75-8 $\quad \mathrm{C}_{20} \mathrm{H}_{14} \quad 254 \quad 1.087$

10.64

9.780

59004-71-6 $\quad \mathrm{C}_{21} \mathrm{H}_{12} \quad 264 \quad 1.124$

11.79

10.48

4.240

83899-23-4 $\quad \mathrm{C}_{21} \mathrm{H}_{12} \quad 264 \quad 1.190$

11.85

9.957

4.241

59004-72-7 $\quad \mathrm{C}_{21} \mathrm{H}_{12} \quad 264 \quad 1.394$

13.56

9.729

83<smiles>C1=CC2C(CC1)CCC1CCC3CC4CCCCC4C3C12</smiles>

$\mathrm{C}_{21} \mathrm{H}_{14}$

266

1.208 
84<smiles>C1=CC2C3CCCCC3C3C4CC5CCCC5CC4C(C1)C23</smiles>

13H-Indeno[1,2-I]phenanthrene

$13 \mathrm{H}$-Dibenzo[a,c]fluorene

7H-Dibenzo[c,g]fluorene

85<smiles>C1CCC2C(C1)CCC1CC3CCCCC3C12</smiles>

86<smiles>C1CCC2C(C1)CC1C2CCC2CCC3CCCCC3C21</smiles>

87<smiles>C1=CC2CC3CCC4CC5CCC=CC5C4C3CC2CC1</smiles>

88<smiles>C1=CC2CCC3CC4CC5CCCCC5CC4C3C2CC1</smiles>

89<smiles>C1=CC2CCC3CC4CC5CCCCC5C4CC3C2CC1</smiles>

$8 H$-Indeno[2,1- $b]$ phenanthrene

13H-Dibenzo[a,g]fluorene 1,2,5,6-Dibenzofluorene

7H-Indeno[1,2-a]phenanthrene

\section{$8 H$-Indeno[1,2-a]anthracene \\ $8 \mathrm{H}$-Naphtho[2,3-c]fluorene}

7H-Dibenzo[b,g]fluorene

90<smiles>C1=CC2CCC3CCCCC3C2CC2CCC3CCCCC3CC12</smiles>

91 1,2,3,4-Dibenzofluorene

201-65-0

$$
\mathrm{C}_{21} \mathrm{H}_{14}
$$

$$
\mathrm{C}_{21} \mathrm{H}_{14}
$$

266

1.466

14.20

198-95-8

$\mathrm{C}_{21} \mathrm{H}_{14}$ 266

1.466

14.43

9.843

204-89-7

$\mathrm{C}_{21} \mathrm{H}_{14}$

266

1.520

14.48

9.525

4.325

241-28-1

$\mathrm{C}_{21} \mathrm{H}_{14}$

266

1.621

15.03

9.272
4.241<smiles>C1CCC2C(C1)CC1C2CCC2CC3CCCCC3C21</smiles>

$\mathrm{C}_{21} \mathrm{H}_{14}$ 266

1.638

15.00 9.160 4.499<smiles>C1=CC2CCC3CCCCC3C2CC2C1CCC1CCCCC12</smiles> 
93<smiles>C1CCC2CC3C(CCC4C5CCCCC5CC34)CC2C1</smiles>

94<smiles>C1CCC2CC3CC4C(CCC5CCCCC54)CC3CC2C1</smiles>

95<smiles>C1CCC2CC3CCC4C5CCCCC5CC4C3CCC2C1</smiles>

96<smiles>C1CCC2CC3C(CC2C1)CC1CC2CCCCC2CC13</smiles>

97<smiles>C1=CC2CCC3C4CC5CCCCC5CC4CC3C2CC1</smiles>

98<smiles>C1CCC2CC3CC4CC5CCCCC5CC4CC3CC2C1</smiles>

99

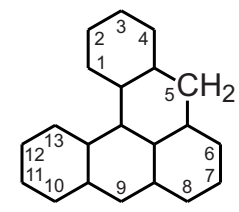

100<smiles>C1=CC2C(C1)CCCC1CC3CCCCC3C1CC1CCCCC12</smiles>

101<smiles>C1=CCC2C3CC4CCCCC4CC3C3CCCC(C1)C23</smiles>

102<smiles>C1=CC2CCCC3C2C(C1)CC1CCC2CCCCC2C13</smiles>

13H-Indeno[2,1-a]anthracene

Benzo[e]cyclopenta[jk]pyrene

13H-Naphtho[2.3-a]fluorene

$12 \mathrm{H}$-Indeno[1,2-b]phenanthrene $12 H$-Naphtho[1,2-b]fluorene 1',2'-Naphtha-2,3-fluorene

11H-Indeno[2,1-a]phenanthrene 11 H-Naphtho[2,1-a]fluorene
2', 1'-Naphtha-1,2-fluorene

12H-Dibenzo[ $b, h]$ fluorene

2,3,6,7-Dibenzofluorene

Di-b-naphthofluorene

242-47-7 $\quad \mathrm{C}_{21} \mathrm{H}_{14}$

266

1.947

16.11

8.278

13H-Dibenzo[a, $h]$ fluorene

$13 H$-Indeno[1,2-b]anthracene

lin-Naphthofluorene

2,3-b-Naphthofluorene

239-85-0

$\mathrm{C}_{21} \mathrm{H}_{14}$

266

1.972

15.83

8.026

4.243

248-93-1

$\mathrm{C}_{21} \mathrm{H}_{14}$

266

2.025

16.14

7.968
4.241

$5 H$-Dibenzo[c,mn]phenanthrene

Ceranthrene

5H-Naphth[3,2,1-de]anthrene

$\mathrm{C}_{21} \mathrm{H}_{14}$

266

1.050

11.93

11.36
5.635

$8 H$-Dibenzo[ $b, m n]$ phenanthrene

8H-meso-a-Naphthanthrene

$\begin{array}{ccc}\mathrm{C}_{21} \mathrm{H}_{14} \quad 266 \quad 1.345 \\ & & 13.97 \\ & & 10.38 \\ & 4.289\end{array}$

$\mathrm{C}_{21} \mathrm{H}_{14} \quad 266 \quad 1.346$

13.92

10.34
4.581

13H-meso-a-Naphthanthrene

7H-Dibenz[a,k]anthracene

$\mathrm{C}_{21} \mathrm{H}_{14} \quad 266$

1.386 
103<smiles>C1=CC2CCC3CC4CCCC5CCCC(C2C1)C4C53</smiles>

4H-Dibenz[a,k]]anthracene

$\mathrm{C}_{21} \mathrm{H}_{14}$

266

1.387

13.00
9.371

5.408<smiles>C1=CCC2C3CCCC4CCC5CCCC6CCC(C1)C2C3C4C65</smiles>

104
$1 H$-Dibenz $[a, k \rrbracket]$ anthracene

Dehydro-8,9-trimethylene-1,2-benzanthrene

4H-Benzo[hi]chrysene

7H-Benzo[hi]chrysene

Naphthobenzanthrene
$194-84-3$

$\mathrm{C}_{21} \mathrm{H}_{14}$

266

1.400

9.21

5.071

105<smiles>C1CCC2C(C1)CC1CCCC3C4CCCC5CCCC(C12)C5C34</smiles>

106<smiles>C1=CC2CCCC3C4CCC5CCCC5C4CC(C1)C23</smiles>

$\mathrm{C}_{21} \mathrm{H}_{14}$ 266

1.495

13.85

9.265

$\mathrm{C}_{21} \mathrm{H}_{14}$

266

1.518

13.96
9.199

9.199
4.219 
107<smiles>C1=CC2C3CCCC4CC5CCC6CCC(C1)C2C3C64C5</smiles>

108

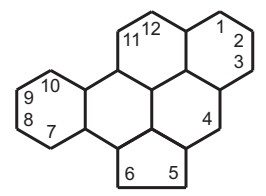

109

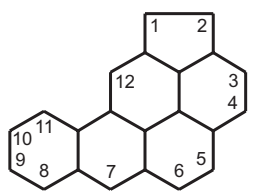

110<smiles>C1=CC2CCC3C(CCC4C5CCCC5CCC43)C2C1</smiles>

111

112<smiles></smiles>

113<smiles>C1=CC2CCC3C4CCCC5C4CCC4CCC(C1)C2C4C53</smiles>

114<smiles>C1CCC2C(C1)C1CCC3C(C1)C2CCC1C2CCCCC2C31</smiles>

Indeno[1,2,3-cd]fluoranthene

Phenanthro[10,1,2,3-cdeffluorene

Benzo[/]cyclopenta[cd]pyrene

Benzo[e]cyclopenta[jk]pyrene

Indeno[5,6,7,1-defg]chrysene

Benzo[def]cyclopenta[qr]chrysene

Cyclopenta[cd]perylene

Aceperylene

Indeno[1,7,6,5-cdef]chrysene

Indeno[1,7-ab]pyrene

Benzo[def]cyclopenta[hi]chrysene

Indeno[1,2,3-cd]pyrene

o-Phenylenepyrene

1,10-(o-Phenylene)pyrene

1, 3 -(o-Ph-Phenylene)pyren

2,3-Phenylenepyrene

115<smiles>C1=CC2CCC3CCC4C5CCCCC5CC(CC1)C2C34</smiles>

189-01-5

$\mathrm{C}_{22} \mathrm{H}_{12}$

276

1.403

12.98
9.256

3.898

196-77-0

$\mathrm{C}_{22} \mathrm{H}_{12}$

276

1.487

13.86

9.323

3.891

193-39-5

$\mathrm{C}_{22} \mathrm{H}_{12}$

276

1.388

13.78

3.884

193-43-1

$\mathrm{C}_{22} \mathrm{H}_{12}$

276

1.617

14.75

9.122

3.889

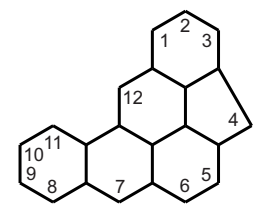

$\mathrm{C}_{22} \mathrm{H}_{12}$

276 
116

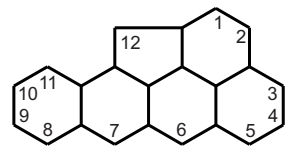

Indeno[6,7,1,2-defg]naphthacene

Naphtho[4,5,6-abc]aceanthrylene

117

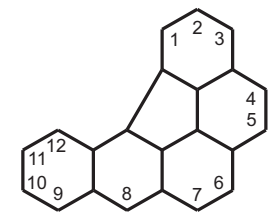

118<smiles>C1=CC2C3CCCCC3C3CCC4CCCC5C4CCCC3C2C5C1</smiles>

119<smiles>C1=CC2CCC3CCC4C5CCCCC5CC(CC1)C4C23</smiles>

120<smiles>C1=CC2CCCC3C4CCCC5CCC(CC1)C2C5C34</smiles>

121

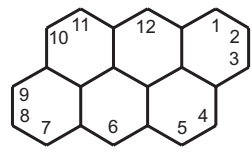

Indeno[3,2,1,7-defg]chrysene

Dibenzo[ $b, m n o]$ fluoranthene

Indeno[4,3,2,1-cdef]chrysene Indeno[7,1,2,3-cdef]chrysene Dibenzo[ $b, g h i]$ fluoranthene

\section{Benzo[ghi]perylene}

1,12-Benzoperylene

Dibenzo[def,mno]chrysene

Anthanthrene

Dibenzo[cd,jk]pyrene
Naphtho[1,2,3,4-ghi]fluoranthene

$\begin{array}{ccc}\mathrm{C}_{22} \mathrm{H}_{12} \quad 276 \quad 1.473 \\ & & 14.15 \\ & 9.609 \\ & 3.885\end{array}$

$\mathrm{C}_{22} \mathrm{H}_{12}$

276

1.246

13.36

10.72

3.895

$\mathrm{C}_{22} \mathrm{H}_{12}$

276

1.269

13.41

10.57 3.883

203-25-8

$\mathrm{C}_{22} \mathrm{H}_{12}$

276

1.393

13.80

9.906 3.889

$191-24-2$

$\mathrm{C}_{22} \mathrm{H}_{12}$

276

1.124

11.78

10.48 3.887

191-26-4 $\quad \mathrm{C}_{22} \mathrm{H}_{12}$

276

1.345 
122<smiles>C1=CC2CCC3CCC4CCCC5C4CCCC5C3C2C1</smiles>

Dibenzo[ $c, g]$ phenanthrene

3,4,5,6-Dibenzophenanthrene gg'-Dibenzophenanthrene

Benzo[b]triphenylene

Dibenz[a,c]anthracene

1,2:3,4-Dibenzanthracene

2,3-Benztriphenylene

Benzo $[g]$ chrysene

Dibenzo[ $[b, g]$ phenanthrene

Naphth[1,2-a]anthracene

Naphtho-2',3',3,4-phenanthrene

Benzo[c]chrysene

1,2,5,6-Dibenzophenanthrene

126<smiles>C1CCC2C(C1)CCC1C2CCC2CCCC3CCCC1C32</smiles>

127<smiles>C1=CC2CCC3CC4CCC5CCCCC5C4CC3C2CC1</smiles>

128<smiles>C1=CC2CC3CCC4CC5CCCCC5CC4C3CC2CC1</smiles>

129<smiles>C1=CC2CCC3CC4CC5CCCCC5CC4CC3C2CC1</smiles>

130<smiles>C1=CC2CCC3CCC4CCCC(C1)C4CC3CC2</smiles>

Dibenz $[a, h]$ anthracene

1,2:5,6-Dibenzoanthracene 1,2:5,6-Benzanthracene 1,2:5,6-Benz[a]anthracene
Pentaphene

Dibenzo $[b, h]$ phenanthrene

2,3,6,7-Dibenzophenanthrene

b, b'-Dibenzophenanthrene

Naphtho-2',3',1,2-anthracene

Benzo[a]naphthacene

1,2,6,7-Dibenzanthracene Naphtho-2',3',2,3-phenanthrene

226-88-0

$\mathrm{C}_{22} \mathrm{H}_{14}$

278

1.801

16.22

9.004

222-93-5

$\mathrm{C}_{22} \mathrm{H}_{14}$

278

1.748

16.09
9.207

3.893

$\begin{array}{llll}\text { 188-52-3 } \quad \mathrm{C}_{22} \mathrm{H}_{14} \quad 278 \quad & 1.165 \\ & & 11.82 \\ & & 10.15 \\ & & 6.240\end{array}$

215-58-7 $\quad \mathrm{C}_{22} \mathrm{H}_{14} \quad 278 \quad 1.238$

13.92

3.889

1.314

13.78

10.49
5.323

195-06-2

$\mathrm{C}_{22} \mathrm{H}_{14}$

278

1.373

13.91

10.13
5.229

$194-69-4$

$\mathrm{C}_{22} \mathrm{H}_{14}$

278

1.519

14.19

5.395

224-41-9

$\mathrm{C}_{22} \mathrm{H}_{14}$

278

1.530

14.54
9.502
3

3.887

53-70-3

$\mathrm{C}_{22} \mathrm{H}_{14}$

278

1.822

15.90

8.726 
<smiles>C1CCC2CC3CCC4CCCCC4C3CCC2C1</smiles>

Benzo $[b]$ chrysene

Benzo[c]tetraphene

2,3:7,8-Dibenzophenanthrene

3,4-Benzotetraphene

Naphth[2,1-a]anthracene

ibenzo[a,h]phenanthrene

Naphtho-2, 3', 1,2-phenanthrene

Naphthanthracene

Picene

1,2:7,8-Dibenzphenanthrene

133<smiles>C1CCC2CC3CC4CC5CCCCC5CC4CC3CC2CC1</smiles>

Pentacene

Benzo[b]naphthacene ,3:6,7-Dibenzanthracene ,3,6,7-Dibenzoanthracene n-Naphthanthracene

,b-Dibenzanthracene

in-Dinaphthanthracene

134<smiles>C1CC2CCC3CCC4CC5CCC6CC1C1C6C5C34C21</smiles>
perylene
214-17-5

$\mathrm{C}_{22} \mathrm{H}_{14}$

278

1.858

16.26

8.755

3.890

213-46-7

$\mathrm{C}_{22} \mathrm{H}_{14}$

278

2.005

16.11

8.037

3.897

$135-48-8$

$\mathrm{C}_{22} \mathrm{H}_{14}$

278

2.226

16.58

7.447

3.885

$1 H$-Benzo[ghi]cyclopenta[pqr]-
64503-02-2 $\quad \mathrm{C}_{23} \mathrm{H}_{12} \quad 288 \quad 1.054$

11.87
11.27

11.27
4.240 


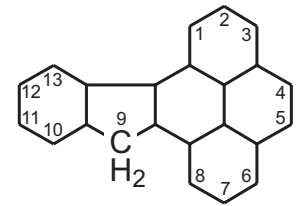

9H-Indeno[1,2-e]pyrene

87308-65-4

$\mathrm{C}_{23} \mathrm{H}_{14}$

290

1.272

13.46
10.58
4.244

4.244

136<smiles>C1CCC2C(C1)CC1CCC3CCC4CCCC4C3CC12</smiles>

7H-Indeno[1,2-a]pyrene

87308-64-3 $\quad \mathrm{C}_{23} \mathrm{H}_{14}$

290

1.557

15.07
9681

9.681
4.242

137

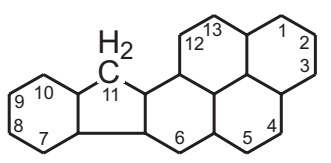

$11 H$-Indeno[2,1-a]pyrene

Indeno-2',3'-3,4-pyrene

196-36-1 $\quad \mathrm{C}_{23} \mathrm{H}_{14}$

290

1.670

15.52

9.290

4.242

138<smiles>C1=CC2CC3CC4CC5CCCCC5C5CCCC(C5)C4C3C2C1</smiles>

$8 H$-Benzo[g]cyclopenta[mno]chrysene

87308-57-4

$\mathrm{C}_{23} \mathrm{H}_{14}$

290

1.213

$8 H$-Benzo[g]cyclopenta[def]chrysene

13.62

4.651

139

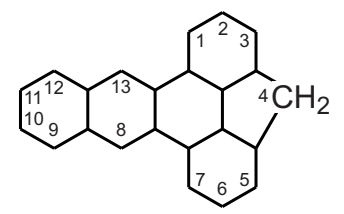

4H-Benzo[b]cyclopenta[jk]triphenylene

$\mathrm{C}_{23} \mathrm{H}_{14}$

290

1.276

13.99

10.96
4.241

140

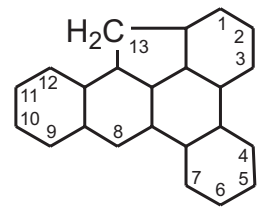

13H-Benzo[b]cyclopenta[def]triphenylene

197-79-5

$\mathrm{C}_{23} \mathrm{H}_{14}$

290

1.290

13.98

10.83

141<smiles>C1CCC2C(C1)CC1CC3C4CCCC1C2C3CCC4</smiles>

4H-Indeno[7,1,2-ghi]chrysene

87308-62-1 $\quad \mathrm{C}_{23} \mathrm{H}_{14}$

$290 \quad 1.325$

13.90

10.50<smiles></smiles>

13H-Dibenz[bc, ] ]aceanthrylene

87308-61-0

$\mathrm{C}_{23} \mathrm{H}_{14}$

290

1.479

14.23

9.620

4.256<smiles>C1=CC2CCC3C(CCC4C5CCCC5CCC43)C2CC1</smiles>

4H-Benzo[c]cyclopenta[mno]chrysene

87308-56-3 $\quad \mathrm{C}_{23} \mathrm{H}_{14}$ 
144

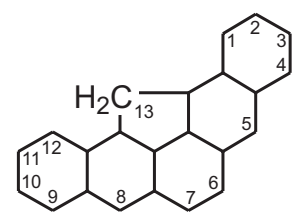

13H-Cyclopenta[rst]pentaphene

13H-Indeno[2,1,7-qra]naphthacene

13H-Dibenz[bc,j]aceanthrylene

$13 \mathrm{H}$-Acenaphtho[1,8-ab]phenanthrene 1',9-Methylene-1,2,5,6-dibenzanthracene

$5 H$-Benzo[b]cyclopenta[def]chrysene

13H-Cyclopenta[pqr]picene

4H-Benzo[b]cyclopenta[mno] chrysene

6H-Cyclopenta[ghi]picene

1,2,7,8-Dibenzo-4,5-phenanthrylenemethane

8H-Dibenzo[b,fg]pyrene

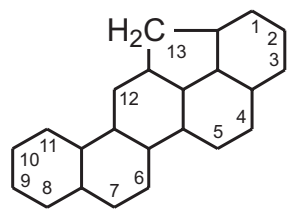

149<smiles>C1=CC2CCCC3CC4CC5CC6CCCCC6CC5C4CC(C1)C23</smiles>

150<smiles>C1CCC2C(C1)CC1CC3CC4CCCCC4C3C3CCC2C13</smiles>

151
87308-59-6

$\mathrm{C}_{23} \mathrm{H}_{14}$

290

1.579

15.34

9.715

87308-63-2 $\quad \mathrm{C}_{23} \mathrm{H}_{14}$

290

1.662

15.55

201-42-3

$\mathrm{C}_{23} \mathrm{H}_{14}$

290

1.709

15.47

9.456
4.242

87308-54-1 $\quad \mathrm{C}_{23} \mathrm{H}_{14}$

290

1.743

15.80

9.065

87308-60-9 $\quad \mathrm{C}_{23} \mathrm{H}_{14}$

$290 \quad 1.786$

15.67

8.771
4.242

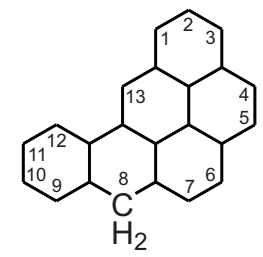<smiles></smiles>

6H-Naphtho[1,2,3-cd]pyrene

1',3'-Naphtha-3,4-pyrene
195-90-4

$\mathrm{C}_{23} \mathrm{H}_{14}$

290

1.815

15.93 8.779
4.242

$\mathrm{C}_{23} \mathrm{H}_{14}$ 290

1.352

13.97 4.341 


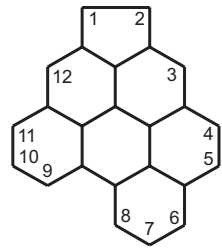

Indeno[5,6,7,1-pqra]perylene

96915-18-3 $\quad \mathrm{C}_{24} \mathrm{H}_{12}$

300

1.096

13.07

3.890

154

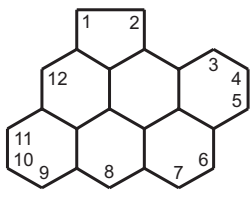

Benz[mno]indeno[5,6,7,1-defg]chrysene

96915-20-7

$\mathrm{C}_{24} \mathrm{H}_{12}$

300

1.196

12.90

10.78

3.890

155<smiles></smiles>

Benzo[ghi]cyclopenta[cd]-

190-88-5

$\mathrm{C}_{24} \mathrm{H}_{12}$

300

1.222

12.83

10.50

perylene

Dibenzo[def,mno]cyclopenta[hi]chrysene

96915-19-4 $\quad \mathrm{C}_{24} \mathrm{H}_{12}$

300

1.305

13.80

10.57

3.890

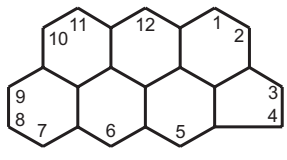

Benz[mno]indeno[1,7,6,5-cdef]chrysene

96915-21-8 $\quad \mathrm{C}_{24} \mathrm{H}_{12}$

300

1.451

13.95

9.613
3.884

3.884

158<smiles>C1CC2CCC3CCCC4CCC5C6CCC(C1)C2C6C5C34</smiles>

Acenaphtho[1,2,3-cde]pyrene

75449-91-1

$\mathrm{C}_{24} \mathrm{H}_{12}$

300

1.275

13.40
10.51

3.893

159<smiles>C1CC2CCC3CCC4CCC5CCC6CCC(C1)C2C6C5C34</smiles>

Coronene

191-07-1

$\mathrm{C}_{24} \mathrm{H}_{12}$

300 
160

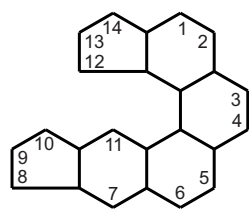

Cyclopent $[b]$ indeno[ $[4,5-g]-$ phenanthrene

Cyclopent $[b]$ indeno[5,6-g]phenanthrene<smiles>C1CC2CCC3CC4C(CCC5CC6CCCC6CC54)CC3CC2C1</smiles>

162<smiles>C1=CC2CCC3CCCCC3C2C2CCCC2C2CCCCC12</smiles>

163<smiles>C1=CC2CC3CC4C5CCCC5C5CCCC5C4CC3CC2CC1</smiles>

164<smiles></smiles>

165<smiles>C1CCC2C(C1)CC1C3CCCCC3C3CC4CCCCC4C3C21</smiles>

166<smiles>C1CCC2C(C1)CC1C2C2CCCCC2C2CC3CCCCC3C21</smiles>

167<smiles>C1=CC2CC3CC4CCC5CC6CCCCC6C5CC4C3CC2CC1</smiles>

168<smiles>C1CCC2C(C1)CC1CCC3C(CCC4CC5CCCCC5C43)C12</smiles>

Dibenzo[c, ]cyclopenta[a]fluorene

Dicyclopenta[a,c]naphthacene

Cyclopent[ $[$ indeno[ $[5,6-a]$ anthracene

Benz[a]indeno[2,1-c]naphthalene

Benz[c]indeno[2,1-a]fluorene

Fluoreno[3,4-b]fluorene

Fluoreno[4,3-c]fluorene
72088-81-4 $\quad \mathrm{C}_{24} \mathrm{H}_{14}$ 302

72088-82-5 $\quad \mathrm{C}_{24} \mathrm{H}_{14}$ 302

$$
\text { 63218-07-5 } \quad \mathrm{C}_{24} \mathrm{H}_{14}
$$

302

1.369

14.98

10.94
4.435

$\begin{array}{llll}15209-78-6 \quad \mathrm{C}_{24} \mathrm{H}_{14} \quad 302 \quad & 1.555 \\ & & & 15.63 \\ & 10.05 \\ & 3.886\end{array}$

222-88-8

$\mathrm{C}_{24} \mathrm{H}_{14}$

302

1.773

16.91

9.537

4.127

193-27-1 $\quad \mathrm{C}_{24} \mathrm{H}_{14} \quad 302 \quad 1.079$

13.39

12.42

4.539

$201-72-9$

$\mathrm{C}_{24} \mathrm{H}_{14}$

302

1.414

15.01

10.62 4.210

198-93-6

$\mathrm{C}_{24} \mathrm{H}_{14}$

302

1.430

15.22
10.64

3.893

$\begin{array}{llll}131238-65-8 & \mathrm{C}_{24} \mathrm{H}_{14} \quad 302 & 1.777 \\ & & 16.43\end{array}$ 
169<smiles>C1CCC2C(C1)CC1CC3CCC4C5CCCCC5CCC4C3CC12</smiles>

Fluoreno[2,3-a]fluorene

223-66-5

$\mathrm{C}_{24} \mathrm{H}_{14}$

302

2.014

17.44
8.658

3.985

170

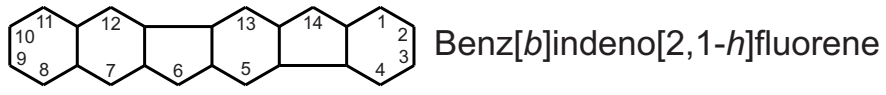

171<smiles>C1CCC2CC3C4CCC5C6CCCCC6C5CCC4C3CC2C1</smiles>

Fluoreno[2,1-a]fluorene

172<smiles>C1CCC2CC3CC4CC5CCCCC5CC4CC3CC2CC1</smiles>

Pentaleno[1,2-b:4,5- $\left.b^{\prime}\right]$ dinaphthalene

173<smiles>C1CCC2C(C1)CC1CC3CC4CC5CCCCC5C4CC3CC12</smiles>

Fluoreno[3,2-b]fluorene

174<smiles></smiles>

Benz[a]indeno[5,6-g]fluorene

as-Indaceno[2,3-a]phenanthrene

Benz[a]indeno[1,2-c]fluorene

Fluoreno[4,3,2-de]anthracene

Anthra[9,1-bc]fluorene

Benz[de]indeno[2,1-b]anthracene

175<smiles></smiles><smiles>C1CCC2C(C1)CC1C2C2CCCCC2C2CC3CCCCC3C21</smiles>

177<smiles>C1=CC2CC3CCCC4C5CCCCC(C(C5)CC2C2CCCCC12)C34</smiles>

178
102634-38-8 $\quad \mathrm{C}_{24} \mathrm{H}_{14}$

302

2.143

17.95

3.389

$221-15-8$

$\mathrm{C}_{24} \mathrm{H}_{14}$

302

2.161

17.28

7.997
3.977

155121-10-1 $\quad \mathrm{C}_{24} \mathrm{H}_{14}$

302

2.234

17.87

3.885

102634-40-2 $\quad \mathrm{C}_{24} \mathrm{H}_{14} \quad 302 \quad 2.266$

18.05

7.964
3.981

$92-89-2$

$\mathrm{C}_{24} \mathrm{H}_{14}$

302

1.626

15.96

4.531

24976-60-1 $\quad \mathrm{C}_{24} \mathrm{H}_{14}$

302

1.958

16.96

8.664
3.943

195-88-0

$\mathrm{C}_{24} \mathrm{H}_{14}$

302

1.521

15.68

10.31
4.451<smiles>C1CCC2C(C1)CC1CC3CC4CCCCC4C3C3CCCC(C3)C12</smiles>

32881-40-6 $\quad \mathrm{C}_{24} \mathrm{H}_{14}$ 
179

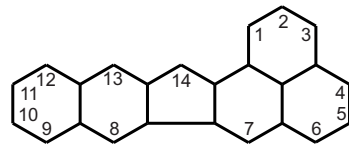

Benz[5,6]indeno[2,1-a]phenalene

Naphth[1,2-a]acephenanthrylene

Indeno[7,1-ab]triphenylene

181<smiles>C1=CC2C3CCCCC(C4CCCCC4CC3C1)C1CCCCC21</smiles>

182<smiles></smiles>

183<smiles>C1CCC2CC3CC4CC5CCCCC5CC4C3CC3CCCCC3CC2C1</smiles>

184

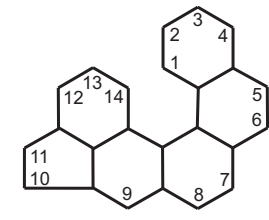

Naphth[2,1-I]acephenanthrylene

Naphth[2,1-e]aceanthrylene

Naphth[2,1-I]aceanthrylene

Benzo[c]cyclopenta[qr]chrysene

Indeno[1,7-ab]triphenylene

Dibenz[a,k]acephenanthrylene

185

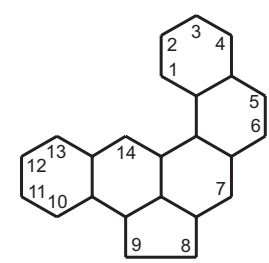

186<smiles></smiles>

$76727-41-8$

$\mathrm{C}_{24} \mathrm{H}_{14}$

302

1.723

15.90

3.889

$\mathrm{C}_{24} \mathrm{H}_{4}$

302

1.243

13.80
11.10 4.898

$\mathrm{C}_{24} \mathrm{H}_{14}$

302

1.250

14.17
11.34

4.713<smiles>C1=CC2CC3C4CCCCC4CC4CCCC(CC4)C3C2C2CCCCC12</smiles>

$\mathrm{C}_{24} \mathrm{H}_{14}$

302

1.342

14.18
10.57 4.920 
188<smiles>C1=CC2C3C=CC4CC5CCCCC5C(CCC3C2C1)C4</smiles>

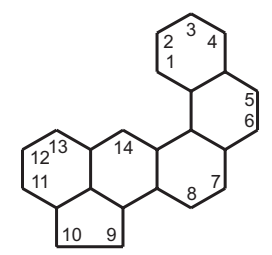

Naphth[1,2-j]aceanthrylene

$\mathrm{C}_{24} \mathrm{H}_{14}$ 302<smiles>C1CCC2C(C1)CCC1CCC3C4CCCC5CCCC(C4)C5C3CC12</smiles>

Benzo[c]cyclopenta[hi]chrysene

$\mathrm{C}_{24} \mathrm{H}_{14}$ 302

1.429

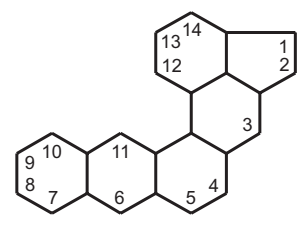

Naphth[2,3-/]acephenanthrylene

$\mathrm{C}_{24} \mathrm{H}_{14}$ 302

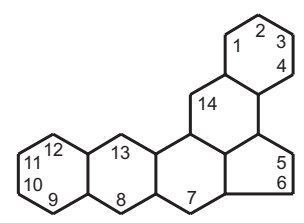

Cyclopenta[fg]pentaphene

$\mathrm{C}_{24} \mathrm{H}_{14}$ 302 1.583 16.16
10.21 3.894

193<smiles>C1=CC2CCCC3CC4CCC5CC6CCCCC6C5C4C3C2C1</smiles>

Cyclopenta[pq]pentaphene

$\mathrm{C}_{24} \mathrm{H}_{14}$ 302<smiles>C1=CC2CC3CCC4C5CC6CCCCC6CC5CC4C3CC2CC1</smiles>

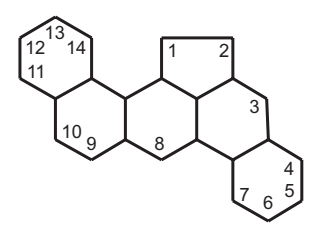

Dibenz[e, ]aceanthrylene

$\mathrm{C}_{24} \mathrm{H}_{14}$<smiles></smiles>

Naphth[2,1-k]acephenanthrylene

$\mathrm{C}_{24} \mathrm{H}_{14}$ 
197<smiles>C1CCC2C(C1)CC1CC3CCC4C5CCCCC5CC4C3CC12</smiles>

Benzo[b]cyclopenta[qr]chrysene

$\mathrm{C}_{24} \mathrm{H}_{14}$

302

1.658

16.27

9.816
3.897

198<smiles>C1=CC2CCC3CC4CC5CCCCC5C4CC3C2CC1</smiles>

Benzo[a]cyclopenta[mn]naphthacene

$\mathrm{C}_{24} \mathrm{H}_{14}$

302

1.709

15.95

9.333

199<smiles>C1=CC2CCC3C4CCC5C(CC6CCCCC65)CC4C3CC2CC1</smiles>

Benzo[a]cyclopenta[fg]naphthacene

$\mathrm{C}_{24} \mathrm{H}_{14}$

302

1.718

16.22

3.442

200<smiles>C1=CC2CCCC3C1CC1C4CCCC5CC(CC23)CC5C1CCC4</smiles>

Naphth[1,2-k]acephenanthrylene Acenaphtho[5,4-b]phenanthrene

201-27-4

$\mathrm{C}_{24} \mathrm{H}_{14}$ 302

1.740

15.89

9.133

201

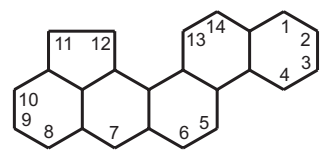

Indeno[7,1-bc]chrysene

$\mathrm{C}_{24} \mathrm{H}_{14}$ 302

1.743

16.07

9.222<smiles>[R]O[R6]</smiles>

Cyclopenta[de]pentaphene

$\mathrm{C}_{24} \mathrm{H}_{14}$ 302

1.745

16.09

9.223

203<smiles>C1=CC2CCC3CC4CC5CCCCC5C5CCC(C5C1)C4C3CC2</smiles>

Benzo[a]cyclopenta[op]naphthacene

$\mathrm{C}_{24} \mathrm{H}_{14}$ 302

1.751

16.10 9.193 3.885

204<smiles>C1=CC2CCC3CC4C5CC6CCCCC6CC5CCC4C(C1)C23</smiles>

Benzo[b]cyclopenta[hi]chrysene

$\mathrm{C}_{24} \mathrm{H}_{14}$ 302<smiles>C1CCC2C(C1)CCC1C2CCC2C3CCCC3CCC21</smiles>

Cyclopenta[de]picene

$\mathrm{C}_{24} \mathrm{H}_{14}$ 302<smiles>C1CCC2C(C1)CCC1C2CCC2C3CCCC3CCC21</smiles> 
<smiles></smiles>

Benzo[a]cyclopenta[hi]naphththacene

$\mathrm{C}_{24} \mathrm{H}_{14}$

302

1.786

16.14

9.037
3.890

208

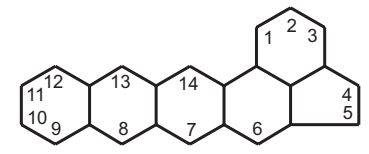

Indeno[7,1-ab]naphthacene

16683-71-9

$\mathrm{C}_{24} \mathrm{H}_{14}$

302

1.800

16.48

9.154

3.885<smiles>C1=CCC2C3CCC4CC(C1)CC(CC1CC5CC=CCC5CC13)C42</smiles>

Cyclopenta[fg]pentacene

$\mathrm{C}_{24} \mathrm{H}_{14}$

302

1.919

16.60

8.650

3.883

210

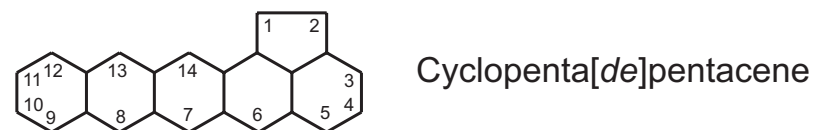

16683-65-1

$\mathrm{C}_{24} \mathrm{H}_{14}$

302

1.919

16.61

8.653

3.886

211<smiles>C1CCC2C(C1)CC1C2CCCC2CC3CCCCC3C21</smiles>

Naphth[1,2-a]aceanthrylene

Dibenzo[a,l]fluoranthene

$\mathrm{C}_{24} \mathrm{H}_{14}$

302

1.121

13.36
11.92

4.942

212<smiles>C1=CC2C3CCCCC3CC3C4CCCCC4C4CC5CCCCC5C4CC23C1</smiles>

Dibenz $[a, e]$ acephenanthrylene Dibenzo[ $b, e]$ fluoranthene

2997-45-7 $\quad \mathrm{C}_{24} \mathrm{H}_{14}$ 302

1.138

13.76

12.09<smiles></smiles>

Dibenzo[j,]fluoranthene

203-18-9

$\mathrm{C}_{24} \mathrm{H}_{14}$

302

1.139

13.12

4.406<smiles>C1CCC2C(C1)CC1C3CCCCC3C3CC4CCCCC4C3C21</smiles>

Dibenz[a,e]aceanthrylene

Dibenzo[a,e]fluoranthene

2,3,5,6-Dibenzofluoranthene

$\begin{array}{llll}5385-75-1 \quad \mathrm{C}_{24} \mathrm{H}_{14} \quad 302 & 1.151 \\ & & & 13.97 \\ & & 2.14 \\ & & 3.895\end{array}$

215<smiles>C1=CC2CCC3CC4CCCC5C6CCCCC6C(C3C2CC1)C45</smiles>

Dibenz[a,] ]aceanthrylene

203-07-6

$\mathrm{C}_{24} \mathrm{H}_{14}$

302

1.183

Naphtho[1,2-a]fluoranthene

1',2'-Naphtho-1,2-fluoranthene 
216<smiles>C1CCC2C(C1)CC1CC3CCCCC3C3C4CCCCC4CC3C12</smiles>

217<smiles>C1CCC2CC3C(CCCC4C5CC6CCCCC6CC5C34)CC2C1</smiles>

218<smiles>C1=CC2CC3CC=CCC3CCC3CC4CCCCC4CC3C2CC1</smiles>

219<smiles>C1=CC2C3CCC4CCC5CCCCC5C4C2C2CCCC1C2C1CCCCC31</smiles>

220<smiles>C1=CC2CC3C(CCC4C5CCCCC5C5CC=CCC5CC34)C2CC1</smiles>

221

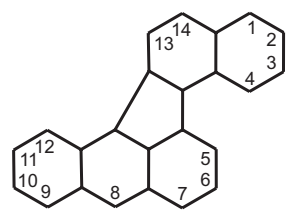

222<smiles>C1=CC2CC3C4CCCC(CC2CC1)C3C1CCCCC41</smiles>

223<smiles>C1CCC2C(C1)C1CCCC3C4CCCCC4C4CC2C1CCCC34</smiles>

Indeno[1,2,3-hi]chrysene

Naphtho[1,2-b]fluoranthene

Naphth[2,1-a]aceanthrylene

Dibbenzo[a,j]fluoranthene

Dibenz[a,j]aceanthrylene

Naphtho[2,1-a]fluoranthene

15,16-Benzodehydrocholanthrene

Naphtho[1,2-j]fluoranthene

224

$\begin{array}{llll}203-11-2 \quad & \mathrm{C}_{24} \mathrm{H}_{14} \quad 302 & 1.269 \\ & & & 14.11 \\ & & 11.13 \\ & 3.969\end{array}$

84030-79-5 $\quad \mathrm{C}_{24} \mathrm{H}_{14}$

302

1.270

14.62

3.886

314-51-2 $\quad \mathrm{C}_{24} \mathrm{H}_{14} \quad 302 \quad 1.320$

14.56

11.03
3.908

$\mathrm{C}_{24} \mathrm{H}_{14}$

302

1.332

13.64

10.24
5.381

Naphth[2,1-e]acephenanthrylene

$\mathrm{C}_{24} \mathrm{H}_{14}$

302

1.380

14.62

10.60
3.910

75519-75-4 $\quad \mathrm{C}_{24} \mathrm{H}_{14} \quad 302 \quad 1.386$

15.03

10.84

4.25

$\begin{array}{llll}203-20-3 \quad \mathrm{C}_{24} \mathrm{H}_{14} \quad 302 \quad & 1.398 \\ & & & 15.07 \\ & 10.78 \\ & & 4.217\end{array}$

111189-32-3 $\quad \mathrm{C}_{24} \mathrm{H}_{14} \quad 302 \quad 1.405$

15.04

15.04
10.71
3.899<smiles>C1CCC2C(C1)CCC1CC3C4CCCCC4C4CCCC(C12)C43</smiles>

Naphtho[2,1-b]fluoranthene
Dibenz[e, ]acephenanthrylene

Naphtho[2,1-b]fluoranthene
Dibenz[e,Iacephenanthrylene 
<smiles>C1=CCC2CC3C4CCCC5C(CCC2C1)C3CC1CCCCC1CC54</smiles>

Naphth[2,3-e]acephenanthrylene

205-97-0

$\mathrm{C}_{24} \mathrm{H}_{14}$

302

1.568

Dibenzo $[b, k]$ fluoranthene

Dibenzo-2,3,11,12-fluoranthene

16.16
10.31

3.898

226<smiles>C1CCC2C3CCCCC3CCCC2C2CCCC3CC(C1)CC32</smiles>

Naphtho[2,3-j]fluoranthene

Acenaphth[1,2-a]anthracene

Naphtho( $\left(2^{\prime}, 3^{\prime}: 7,8\right)$ fluoranthene

205-83-4

$\mathrm{C}_{24} \mathrm{H}_{14}$

302

1.581

15.51

9.810

227<smiles>C1=CC2CCC3C4CCCC5CC6CCCCC6C5CCC(C4C1)C23</smiles>

Naphth[1,2-e]acephenanthrylene

Dibenzo[b,j]fluoranthene

$5385-22-8$

$\mathrm{C}_{24} \mathrm{H}_{14}$

302

1.597

15.93

9.973
3.901

228<smiles></smiles>

Dibenz $[e, k]$ acephenanthrylene

Naphtho[2,3-b]fluoranthene

Naphtho[2', 3':2, 3]fluoranthene

206-06-4

$\mathrm{C}_{24} \mathrm{H}_{14}$

302

1.608

16.20
10.07
3.889

3.889

229<smiles>C1=CC2CCC3CC4C5CC3C(CCCC2C1)C4CCCC1CCCC5C1</smiles>

Naphtho[1,2-k]fluoranthene

Acenaphtho[1,2-b]phenanthrene

238-04-0

$\mathrm{C}_{24} \mathrm{H}_{14}$

302

1.638

15.52

3.973

230<smiles></smiles>

Naphtho[2,1-j]fluoranthene

$\mathrm{C}_{24} \mathrm{H}_{14}$

302

1.641

15.70

9.567

4.386

231

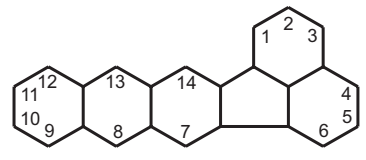

Naphtho[2,3-k]fluoranthene

Acenaphth[1,2-b]anthracene Naphtho $\left(2^{\prime}, 3^{\prime}: 8,9\right)$ fluoranthene

207-18-1

$\mathrm{C}_{24} \mathrm{H}_{14}$

302

1.740

16.07

9.237
3.895

232<smiles>C1CCC2CC3CCCCC3C3C4CCCCC4C(CC1)C23</smiles>

Dibenzo[def,p]chrysene

Dibenzo[a,/]pyrene

3,4:8,9-Dibenzopyrene

2,3:4,5-Dibenzpyrene

4,5,6,7-Dibenzpyrene

1,2:9,10-Dibenzopyrene

191-30-0

$\mathrm{C}_{24} \mathrm{H}_{14}$

302

1.171

13.69

11.70
5.167

233

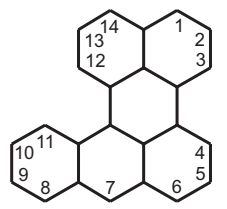

Benzo[a]perylene

191-85-5

$\mathrm{C}_{24} \mathrm{H}_{14}$

302

1.177

13.65

1,2-Benzoperylene

1,2-Benzperylene

5.469

Naphtho[1,2,3,4-def]chrysene

192-65-4

$\mathrm{C}_{24} \mathrm{H}_{14}$

302 
<smiles></smiles>

Dibenzo[de,qr]naphthacene

Naphtho[2,3-e]pyrene

Dibenzo $d e, q r]$ tetracer<smiles>C1CCC2C(C1)C1CCCC3C4CCCC5CCC(C1)C2C5C34</smiles>

236

Naphtho[8,1,2-ghi]chrysene

Naphtho[1,2-e]pyrene

Dibenzo[fg,op]naphthacene

Dibenzo[e,/]pyrene

4,5,9,10-Dibenzopyrene<smiles>C1CCC2C(C1)C1CCCCC1C1CCCCC1C1CCCCC21</smiles>

238<smiles></smiles>

239<smiles></smiles>

240<smiles></smiles>

241

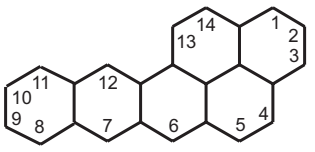

Naphtho[2,1,8-qra]naphthacene

Naphtho[2,3-a]pyrene

Naphtho[2.3', 1.2$]$ pyrene

Naphtho 2,3-b]pyrene

Naphtho[8,1,2-cde]naphthacene

\section{2,3-Benzoperylene \\ Dibenzo[de,op]naphthacene}

Dibenzo[c,mno]chrysene

Naphtho[1,2-a]pyrene

Dibenzo[de,mn]naphthacene

Zethrene<smiles></smiles>

243<smiles>C1=CCC2C(C1)CC1CCC3CC4CCCCC4C4CCC(C12)C34</smiles><smiles></smiles>

Naphtho[3', $\left.4^{\prime}: 3,4\right]$ pyrene

Benzo[rst]pentaphene

Dibenzo[a,i]pyrene

Dibenzo[b, $h]$ pyrene

3,4:9,10-Dibenzopyrene

1,2:7,8-Dibenzpyrene

4,5,8,9-Dibenzopyrene

Dibenzo[b,def]chrysene
193-09-9

$\mathrm{C}_{24} \mathrm{H}_{14}$

302

1.288

14.37
11.16

4.411

35699-67-3 $\quad \mathrm{C}_{24} \mathrm{H}_{14} \quad 302 \quad 1.323$

13.82

10.45
5.150

$192-51-8$

$\mathrm{C}_{24} \mathrm{H}_{14}$

302

1.330

13.98

10.51
3.886

197-70-6

$\mathrm{C}_{24} \mathrm{H}_{14}$

302

1.396

14.38

10.30
4.844

196-28-1 $\quad \mathrm{C}_{24} \mathrm{H}_{14} \quad 302 \quad 1.519$

14.37

9.461

5.133

$\begin{array}{llll}\text { 214-63-1 } \quad \mathrm{C}_{24} \mathrm{H}_{14} & 302 & 1.532 \\ & & 14.39 \\ & & 9.393 \\ & & 4.894\end{array}$

196-42-9 $\quad \mathrm{C}_{24} \mathrm{H}_{14} \quad 302 \quad 1.693$

16.23

9.587

3.890

189-96-8 $\quad \mathrm{C}_{24} \mathrm{H}_{14} \quad 302 \quad 1.723$

16.02

9.300

4.187

189-55-9 $\quad \mathrm{C}_{24} \mathrm{H}_{14} \quad 302 \quad 1.732$

16.09

9.291
3.893

$189-64-0$

$\mathrm{C}_{24} \mathrm{H}_{14}$

302

1.732 
245<smiles>C1CCC2CC3C(CC2C1)CC1CCC2CCC4CCCCC4C2C13</smiles>

9H-Benz[5,6]indeno[2,1-c]-

phenanthrene

$\mathrm{C}_{25} \mathrm{H}_{16}$

316

1.266

14.12

11.15
5.528

15H-Benz[4,5]indeno[1,2-I]-

phenanthrene

$\mathrm{C}_{25} \mathrm{H}_{16}$

316

1.322

15.05
11.38

4.249

10H-Benz[g]indeno[2,1-a]-

phenanthrene

$\mathrm{C}_{25} \mathrm{H}_{16}$

316

1.344

14.36

14.36
10.68
5.467

$\mathrm{H}_{2}$

248<smiles>C1CCC2C(C1)CC1CCC3C4CCCCC4C4CCCCC4C3C12</smiles>

$11 H$-Indeno[1,2-a]triphenylene 3,4-(o,o'-Biphenylene)-fluorene

9H-Benz[4,5]indeno[2,1-c]phenanthrene

$\mathrm{C}_{25} \mathrm{H}_{16}$ 316

1.440

14.76

10.76
5.436<smiles>CC1CCCCC1C1CCCC1</smiles>

250<smiles>C1CCC2C(C1)CCC1C2CCC2CC3CCCCC3C21</smiles>

5H-Indeno[2,1-a]chrysene

7H-Benz[5,6]indeno[1,2-a]phenanthrene

$\mathrm{C}_{25} \mathrm{H}_{16}$

316 1.767 16.93 9.584 4.606
252<smiles>C1=CC2CC3CCC4C5CCCCC5CCC4C2CCC3C1</smiles>

7H-Benz[5,6]indeno[2,1-a]phenanthrene

$\mathrm{C}_{25} \mathrm{H}_{16}$ 
<smiles>C1CCC2CC3C(CC4CCCCC43)CC3C4CCCCC4CC(C1)CC23</smiles>

$\mathrm{C}_{25} \mathrm{H}_{16}$

316

1.510

16.13
10.69

10.69
5.275

254

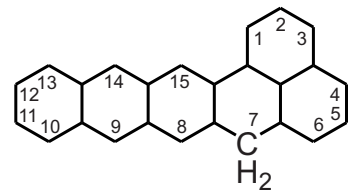

$7 H$-Benzo[de]pentacene

$\mathrm{C}_{25} \mathrm{H}_{16}$

316

1.764

16.83

9.541
4.647 
<smiles>C1CC2CCCC3C(CCCC4C5CCCC6C7CCCC(C56)C7C34)C2C1</smiles>

Rubicene

Diindeno[1,2,3-de,1',2',3',k] ]anthracene

Acenaphtho[1,2-j]fluoranthene

256<smiles>C1CC2CCCC3C2C(C1)C1CCCC2C4CCCC5CCCC(C52)C4C13</smiles>

257

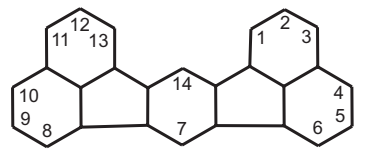

258

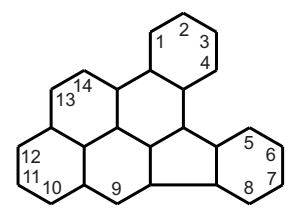<smiles>C1CCC2C(C1)CC1CCC3CC4C5CCCCC5C5CCC(C1)C2C3C54</smiles>

Acenaphtho[1,2-k]fluoranthene Benzo[1,2-a,4,5-a']diacenaphthylene 3,4,1,6-Di(1,8-naphthylene)benzene

Fluoreno[3,2,1,9-defg]chrysene

Benz[def]indeno[1,2,3-qr]chrysene

Benz[def]indeno[1,2,3-hi]chrysene

Indeno[1,2,3-cd]perylene

Dibenzo[a,ghi]perylene

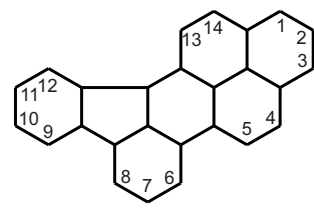

261<smiles></smiles>

$197-61-5$

$\mathrm{C}_{26} \mathrm{H}_{14}$

326

1.387

14.90
10.75

3.893

$\begin{array}{ccc}193-21-5 \quad \mathrm{C}_{26} \mathrm{H}_{14} \quad 326 \quad & 1.517 \\ & & 15.17 \\ & & 10.00 \\ & 4.632\end{array}$

207-02-3 $\quad \mathrm{C}_{26} \mathrm{H}_{14}$

326

1.666

15.42
9.257

3.888

192-35-8 $\quad \mathrm{C}_{26} \mathrm{H}_{14} \quad 326 \quad 1.128$

13.79

12.22
3.951

260

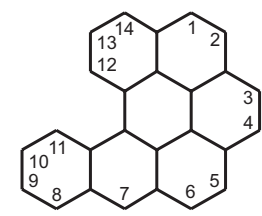

111189-34-5 $\quad \mathrm{C}_{26} \mathrm{H}_{14}$

326

1.356

15.14

11.17

$\begin{array}{llll}111189-33-4 \quad \mathrm{C}_{26} \mathrm{H}_{14} \quad 326 \quad & 1.400 \\ & & & 15.08 \\ & & 10.77 \\ & 3.896\end{array}$

101686-49-1 $\mathrm{C}_{26} \mathrm{H}_{14} \quad 326 \quad 1.667$ 15.43
9.257 3.960

6596-37-8 $\quad \mathrm{C}_{26} \mathrm{H}_{14} \quad 326 \quad 1.180$ 
263

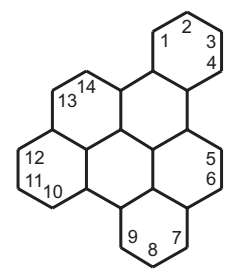

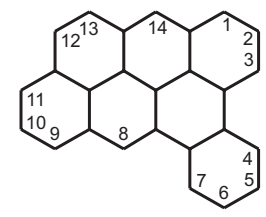

Benzo[qr]naphtho[3,2,1,8-defg]chrysene Benzo[e]anthanthrene

\section{Dibenzo[b,ghi]perylene}

1.12,4.5-Dibenzoperylene

Dibenzo[b,pqr]perylene 1.12,2.3-Dibenzoperylene Dibenzo[e,ghi]perylene

Naphtho[8,1,2-bcd]perylene

Naphtho[7,8,1,2,3-pqrst]pentaphene Benzo[a]anthanthrene

\section{Anthra[2,1,9,8-opqra]naphthacene}

Benz[b]anthanthrene
5869-30-7 $\quad \mathrm{C}_{26} \mathrm{H}_{14}$

$190-95-4$

$\mathrm{C}_{26} \mathrm{H}_{14}$

$188-89-6$

$\mathrm{C}_{26} \mathrm{H}_{14}$

326

120835-55-4 $\quad \mathrm{C}_{26} \mathrm{H}_{14}$ 326

1.520

16.01

10.53

269

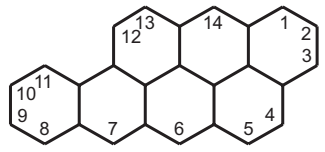

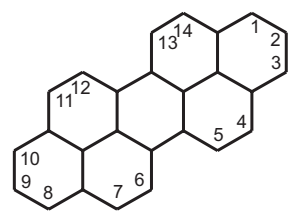


<smiles>C1CC2CCC3CCC4CCC5CCC6CCC(C1)C2C6C5C34</smiles>

Phenanthro[3,4-c]phenanthrene

Hexahelicene

[6] Helicene

Dibenzo[g,p]chrysene

1,2:3,4:5,6:7,8-Tetrabenzonaphthalene Dibenzo[a,c]triphenylene Biphenylenephenanthrene

9,10-Diphenylenephenanthrene Difluorenylene

273<smiles>C1CCC2C(C1)CC1C2CC2C3CCCCC3CC2C2CCCCC12</smiles>

Naphtho[2,3-g]chrysene

Dibenz[a, $n]$ triphenylene Dibenzo[a,f]tetraphene

Phenanthro[4,3-a]anthracene

Naphtho[1,2-a]tetraphene

Benzo[b]pentahelicene

Naphtho[1,2-g]chrysene

Benzo[i]pentahelicene

Dibenzo[c,p]chrysene

Anthra[1,2-a]anthracene

Naphtho[2,3-a]tetraphene

Anthraceno-1',2', 1,2-anthracene<smiles>C1CC2CCC3CC4CCCCC4CC3CC2C1</smiles>

187-83-7 $\quad \mathrm{C}_{26} \mathrm{H}_{16}$

328

1.074

11.86

7.413

191-68-4

$\mathrm{C}_{26} \mathrm{H}_{16}$

328

1.095

13.55

12.38
6.154

196-64-5

$\mathrm{C}_{26} \mathrm{H}_{16}$

328

1.107

13.89
1254

5.432

$\begin{array}{llll}58029-40-6 & \mathrm{C}_{26} \mathrm{H}_{16} & 328 & 1.162 \\ & & & 13.79 \\ & & 11.87 \\ & 6.238\end{array}$

191-67-3 $\quad \mathrm{C}_{26} \mathrm{H}_{16}$

328

1.184

13.74

13.60
11.60

11.60
6.107

$\begin{array}{lll}196-52-1 \quad \mathrm{C}_{26} \mathrm{H}_{16} \quad 328 \quad & 1.215 \\ & & 14.13 \\ & & 11.62 \\ & 5.615\end{array}$

195-00-6

$\mathrm{C}_{26} \mathrm{H}_{16}$

328

1.291

14.68

14.68
5.231
Naphtho[2,1-c]chrysene

Benzo[c]pentahelicene
58029-44-0

$\mathrm{C}_{26} \mathrm{H}_{16}$

328 
<smiles>C1CCC2CC3C(CCC4CCC5CCCCC5C43)CC2C1</smiles>

Benzo[a]naphth[2,1-j]anthracene

Dibenzo[a,m]tetraphene

Dibenzo[c,g]chrysene

Benzo[f]pentahelicene

Benzo[ $h]$ pentaphene

Dibenzo[a,c]naphthacene

Naphtho[1,2-b]triphenylene

Tribenzo[a,c, $h]$ anthracene

Dibenzo[ $f, m]$ tetraphene

1,2,3,4,5,6-Tribenzanthracene

284<smiles>C1=CCC2CC3C(CCC2C1)C1CCCCC1C1C2CCCCC2CCC31</smiles>

Dibenzo[ $[b, p]$ chrysene

Dibnezo[c,f]tetraphene

Naphtho[2,3-c]chrysene

Naphtho[2,1-a]tetraphene

Dibenzo[a,c]tetraphene

Dibenzo $[b, g]$ chrysene

285<smiles>C1=CC2CC3C(CCC2CC1)CCC1CCC2CCCCC2CC13</smiles>

286
58029-41-7 $\quad \mathrm{C}_{26} \mathrm{H}_{16}$ 328

1.311

14.43

11.00 5.246

53156-66-4 $\quad \mathrm{C}_{26} \mathrm{H}_{16}$ 328

1.334

13.85

10.38

6.569

$\mathrm{C}_{26} \mathrm{H}_{16}$

328

1.359

15.90

11.70

4.850

$\begin{array}{llll}216-00-2 & \mathrm{C}_{26} \mathrm{H}_{16} \quad 328 \quad & 1.410 \\ & & & 16.19 \\ & & 11.48 \\ & & 3.891\end{array}$

215-26-9

$\mathrm{C}_{26} \mathrm{H}_{16}$

328

1.414

15.89

11.24

4.138

58029-42-8 $\quad \mathrm{C}_{26} \mathrm{H}_{16} \quad 328 \quad 1.442$

16.13

11.19<smiles>C1CCC2CC3CC4CCCCC4C4CCCCC4C3CCC2C1</smiles>

58029-37-1 $\quad \mathrm{C}_{26} \mathrm{H}_{16}$

328

1.516

16.14

10.64
5.444

53156-67-5 $\quad \mathrm{C}_{26} \mathrm{H}_{16} \quad 328 \quad 1.522$ 
<smiles>C1CCC2C(C1)CCC1C2CCC2C3CCCCC3C3CCCCC3C12</smiles>

287

Benzo[f]picene

Dibenzo[a,c]chrysene

1.2,5.6-Dibenzotetraphene

Benzo[s]picene

Dibenzo[a,p]chrysene

288<smiles>C1=CC2CCC3C(CC2CC1)C1CCCCC1C1C2CCCCC2CCC31</smiles>

289<smiles>C1CCC2CC3C(CCC4CC5CCCCC5CC43)CC2C1</smiles>

290<smiles>C1CCC2CCC3CC4CC5CCCCC5CC4CC3CC2CC1</smiles>

291

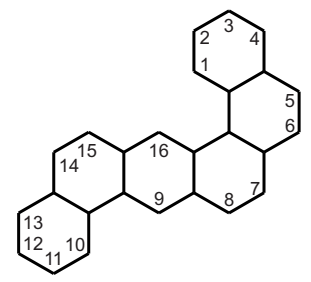

Naphtho[1,2-a]naphthacene

Naphtho[1,2-a]tetracene

Benzo[a]naphtho[1,2-h]anthracene

dibenzo[a,k]tetraphene

Benzo[a]picene

Dibenzo[c, ]chrysene

Naphtho[1,2-c]chrysene<smiles>C1CCC2C(C1)CCC1C2CCC2C3CCCCC3CCC21</smiles>

293<smiles>C1CCC2CC3C(CCC2C1)CCC1C2CCCCC2CCC31</smiles>

58029-47-3 $\quad \mathrm{C}_{26} \mathrm{H}_{16}$

328

1.523

15.94
10.47

5.442

31540-94-0

$\mathrm{C}_{26} \mathrm{H}_{16}$

328

1.528

15.75

5.997

$\begin{array}{cccc}7689-57-8 \quad \mathrm{C}_{26} \mathrm{H}_{16} \quad 328 \quad & 1.531 \\ & & 16.42 \\ & & 10.72 \\ & 3.904\end{array}$

58029-39-3

$\mathrm{C}_{26} \mathrm{H}_{16}$

328

1.555

16.12

10.37
4.999

292<smiles>C1CCC2C(C1)CCC1C2CCC2CCC3C4CCCC3C(CCC4)C21</smiles>

$18429-26-0$

$\mathrm{C}_{26} \mathrm{H}_{16}$

328

1.635

16.73

10.23
3.899

58029-45-11 $\quad \mathrm{C}_{26} \mathrm{H}_{16} \quad 328 \quad 1.636$

16.29

9.958

5.267

$\mathrm{C}_{26} \mathrm{H}_{16}$

328

1.679

15.46

9.411
5.965

58029-46-2 $\quad \mathrm{C}_{26} \mathrm{H}_{16} \quad 328 \quad 1.761$ 
<smiles>C1CCC2C(C1)CC1CC3CCC4C5CCCCC5CCC4C3CC12</smiles>

Naphtho[2,1-b]chrysene Dibenzo[c, $m]$ tetraphene

$$
\text { 58029-43-9 } \quad \mathrm{C}_{26} \mathrm{H}_{16}
$$

328

1.777

16.94

9.532

296<smiles>C1CCC2CC3CCC4C(CCC5CCC6CCCCC6C54)C3CCC2C1</smiles>

Dibenzo[ $b, /]$ chrysene

Naphtho[2,1-c]tetraphene Phenanthro[3,4-a]anthracene

58029-38-2 $\quad \mathrm{C}_{26} \mathrm{H}_{16}$<smiles>[10BH2][20BH3-]</smiles>

Dibenzo[a,/]naphthacene

1.2,9.10-Dibenzotetracene 2, 3'-Phenanthra-2,3-phenanthrene

298

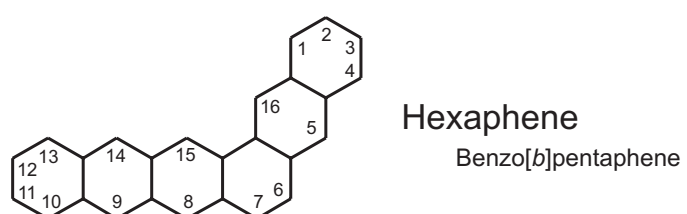

299<smiles>C1=CC2CC3CCC4CC5CCCCC5CC4CC3CCC2C1</smiles>

Benzo[c]pentaphene

Anthra[1,2-b]phenanthrene 2',3'-Phenanthra-1,2-anthracene

300<smiles>C1CCC2C(C1)CCC1CC3CC4CC5CCCCC5C4CC3CC12</smiles>

\section{Dibenzo[a,]naphthacene}

1.2,7.8-Dibenzotetracen Dibenzo[a,j]tetracene

301<smiles>C1CCC2CC3CC4CC5CCCCC5CC4CC3CC2C1</smiles>

Benzo[a]pentacene

Benzo[b]picene

2,3-Benzopicene<smiles>CC1CCC2C1CCC1C2CCC2C3CCC4CC5CCCCC5CC4C3CCC12</smiles><smiles>C1=CCC2CC3CC4C5CC6CCCCC6CC5CCC(C2CC1)C4C3</smiles>

Naphtho[2,1-a]naphthacene 
<smiles>C1CCC2C(C1)CC1CC3CCC4C5CCCCC5CCC4C3CC12</smiles>

Naphtho[1,2-b]chrysene

Dibenzo[c,k]tetraphene

Dibenzo $[b, k]$ chrysene

Anthraceno[2,1-a]anthracene

Benzo[c]picene

Hexacene

2,3-Benzo-6,7-naphthoanthracene

258-31-1

$\mathrm{C}_{26} \mathrm{H}_{16}$

328

2.543

19.05

7.493

217-54-9

$217-37-8$

$\mathrm{C}_{26} \mathrm{H}_{16}$

328

2.274

18.30 8.048
3.899

308

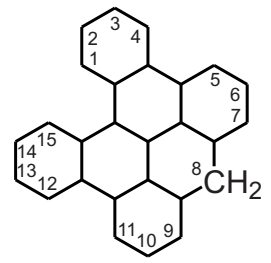

309

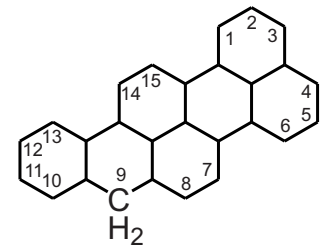

8H-Tribenzo[a,cd,/]pyrene

[Phenanthreno-9',10':9,10]phenanthrene-1,1'-methylene

9H-Naphtho[1,2,3-cd]perylene
$\mathrm{C}_{27} \mathrm{H}_{16}$

340

1.073

13.66 12.73
5.357

$\mathrm{C}_{27} \mathrm{H}_{16}$ 
310<smiles>C1CCC2C(C1)CC1C3CCCCC3C1C1CC3CCCCC3C21</smiles>

Truxene

10,15-Dihydrotribenzo[a,f,k]trindene Triindeno[2,3:2', 3':2", $\left.3^{\prime \prime}\right]$ benzene

Diindeno[1,2,3-cd:1',2',3'-jk]pyrene

311

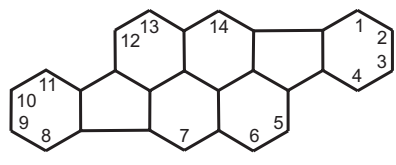

312

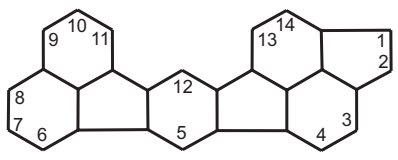

Acenaphtho[1,2-k]cyclopenta[cd]fluoranthene

313

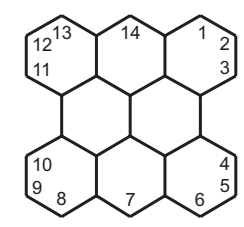

314<smiles></smiles>

315<smiles></smiles>

Benzo[a]coronene

Phenanthro[5,4,3,2-abcde]perylene

Bisanthrene

Dibenzo[fg,op]anthanthrene

Benzo[cd]naphtho[3,2,1,8-pqra]perylene

Naphtho[8,1,2-efg]anthanthrene Phenaleno[12,3,4-ghi]perylene

316
Dibenzo[cd,fg]anthanthrene

$548-35-6$

$\mathrm{C}_{27} \mathrm{H}_{18}$

342

1.064

14.43

4.256

191-23-1 $\quad \mathrm{C}_{28} \mathrm{H}_{14}$

350

1.728

17.13

9.917

30909-04-7 $\quad \mathrm{C}_{28} \mathrm{H}_{14}$

350

1.803

16.66

9.241

3.888

Phenanthro[1,10,9,8-opqra]perylene

190-39-6

$\mathrm{C}_{28} \mathrm{H}_{14}$

350

1.064

13.83

13.00

3.892

6208-20-4 $\quad \mathrm{C}_{28} \mathrm{H}_{14}$

350

1.183

13.90

11.76
3.893

190-70-5 $\quad \mathrm{C}_{28} \mathrm{H}_{14} \quad 350 \quad 1.215$

14.23

11.71

3.888<smiles></smiles>

75449-92-2 $\quad \mathrm{C}_{28} \mathrm{H}_{14}$

350 


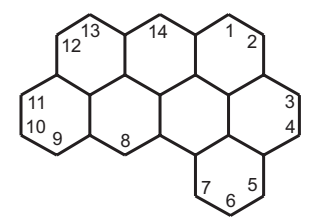

Benzo[Imn]naphtho[2,1,8-qra]perylene Dibenzo[cd,hi]anthanthrene

Benzo[pqr]naphtho[8,1,2,-bcd]perylene

Dibenzo[hi,qr]anthanthrene

319

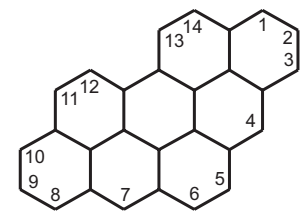

320<smiles>C1CC2CC3CC4CCC5CCCC(CC6CC7CCC(C1)C6C2CC7)C5C43</smiles>

321<smiles></smiles>

322<smiles>C1CCC2CC3C(CCCC4C5CCC6CC7CCCCC7CC6C5C34)CC2C1</smiles>

Anthra[1,2-a]aceanthrylene

Anthra[2,1-a]aceanthrylene

Dibenzo[a,o]perylene

o-meso-Benzodianthrene

peri-Naphthacenonaphthacene

Dibenzo[cd,Im]anthanthrene

Naphthaceno[4,5,6,7,8-defghij]naphthacene

Fluoreno[9,1-ab]triphenylene

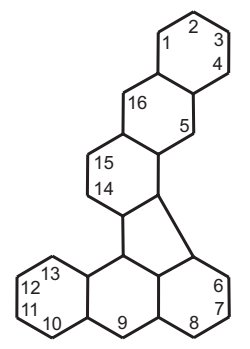

$\begin{array}{llll}\text { 75449-94-4 } \quad \mathrm{C}_{28} \mathrm{H}_{14} \quad 350 \quad & 1.290 \\ & & & 14.39 \\ & 11.15 \\ & 3.887\end{array}$

190-71-6 $\quad \mathrm{C}_{28} \mathrm{H}_{14}$ 350

1.331

13.97

10.50

4.26

4552-79-8 $\quad \mathrm{C}_{28} \mathrm{H}_{14} \quad 350 \quad 1.511$

15.96

10.56
3.894

180-50-1 $\quad \mathrm{C}_{28} \mathrm{H}_{14} \quad 350 \quad 1.599$

15.35

9.600
3.885

136276-45-4 $\quad \mathrm{C}_{28} \mathrm{H}_{16}$

352

1.254

13.72

10.94
6.082

323<smiles>C1CCC2C(C1)CC1CCCC3C4CCCC5CC6CCCCC6C(C12)C5C34</smiles>

203-06-5

$\mathrm{C}_{28} \mathrm{H}_{16}$

352

1.277

15.23

11.92
5.182

$\mathrm{C}_{28} \mathrm{H}_{16}$

352

1.604

17.40
10.85

10.85
4.426 
325

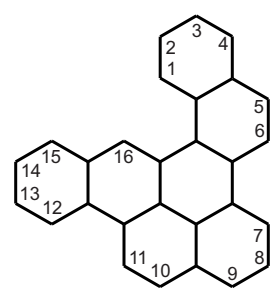

Phenanthro[1,2,3,4-def]chrysene

Benzo[fg]naphtho[1,2,3-op]-

naphthacene

Benzo[p]naphtho[1,8,7-ghi]chrysene

Dibenzo[a,f]perylene

Tribenzo[c,g,mno]chrysene

Dibenzo[a,n]perylene

330<smiles></smiles>

331<smiles></smiles>

Benzo[de]naphtho[3,2,1-mn]naphthacene

Benzo[mno]naphtho[2,1-c]chrysene
137570-58-2 $\quad \mathrm{C}_{28} \mathrm{H}_{16}$

352

1.101

13.85

12.58

137570-59-3 $\quad \mathrm{C}_{28} \mathrm{H}_{16} \quad 352 \quad 1.157$

13.75 11.88
5.780

385-14-8 $\quad \mathrm{C}_{28} \mathrm{H}_{16}$ 352

1.166

13.02
11.17

11.17
6.251

191-29-7 $\quad \mathrm{C}_{28} \mathrm{H}_{16} \quad 352 \quad 1.187$ 13.92
11.73
5.318

$\begin{array}{cccc}108650-10-8 & \mathrm{C}_{28} \mathrm{H}_{16} \quad 352 & 1.222 \\ & & & 14.19 \\ & 11.61 \\ & 6.394\end{array}$

191-81-1 $\quad \mathrm{C}_{28} \mathrm{H}_{16}$ 352 1.226 13.97
11.40 5.836<smiles></smiles>

137570-57-1 $\quad \mathrm{C}_{28} \mathrm{H}_{16}$ 
<smiles>C1CCC2C(C1)CCC1CCC3C4CCCC5CCCC6CCCC(C54)C3C2C1C6</smiles><smiles>C1CCC2C(C1)CC1C3CCCCC3C3C4CCCCC4CC4CCC2C1C43</smiles><smiles>C1CCC2CC3CC4CCCCC4CC3C3CC2C(CC1)C1CCCCC31</smiles><smiles>C1CCC2C(C1)CC1C3CCCCC3C3CC4CCCCC4C4CCC2C1C43</smiles>

Dibenzo[h,rst]pentaphene

$192-47-2$

$\mathrm{C}_{28} \mathrm{H}_{16}$

352

1.363

16.06
11.79

4.711

337<smiles>C1CCC2C(C1)CC1CCCC3C4C5CCCCC5CC5CCCC(C12)C5C34</smiles>

Dibenzo[a,j]perylene p-meso-Benzodianthrene

191-87-7 $\quad \mathrm{C}_{28} \mathrm{H}_{16}$ 352

15.62

11.46<smiles>[13CH3]</smiles>

Naphtho[2,1,8-fgh]pentaphene

19301-88-3

$\mathrm{C}_{28} \mathrm{H}_{16}$

352

1.364

16.00
11.73

11.73
4.413

339

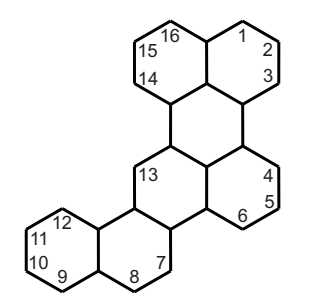

Naphtho[1,2-b]perylene

$120835-41-8$

$\mathrm{C}_{28} \mathrm{H}_{16}$

352

1.367

16.00

11.70<smiles></smiles> 


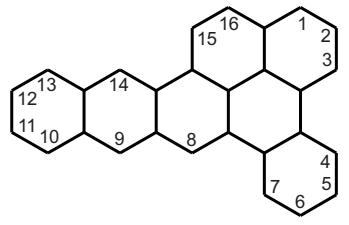

Benzo[a]naphtho[8,1,2-cde]-

naphthacene

192-70-1

$\mathrm{C}_{28} \mathrm{H}_{16}$

352

1.406

16.12

11.47
4.365

Dibenzo[f,pqr]picene

120835-42-9

$\mathrm{C}_{28} \mathrm{H}_{16}$

352

1.434

15.64
10.91

5.746

343<smiles>C1CCC2C(C1)CC1C3CCC4CCCCC4C3C3CCCC4CCCC2C4C13</smiles>

Benzo[b]naphtho[8,1,2-pqr]chrysene

Phenanthro[4,3,2,1-def $]$ chrysene

137593-96-5

$\mathrm{C}_{28} \mathrm{H}_{16}$

352

1.444

16.12

11.16

344

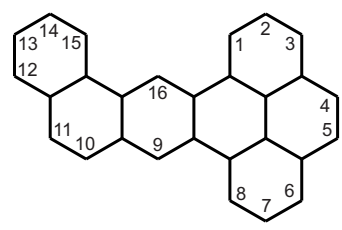

Tribenzo[a,hi,mn]naphthacene

Dibenzo[de,uv]pentacene

193-11-3

$\mathrm{C}_{28} \mathrm{H}_{16}$

352

1.478

16.87

11.42

3.894

54961-30-7 $\quad \mathrm{C}_{28} \mathrm{H}_{16} \quad 352 \quad 1.462$

16.38
11.20

4.192

345<smiles>CC1CCC2CCCC3C4CC5CC6CCCCC6CC5CC4C(C)C1C23</smiles>

346

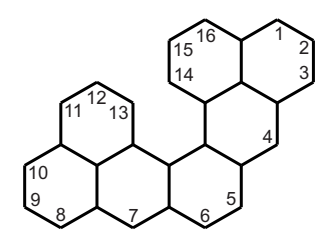

Dibenzo[pq,uv]pentaphene

Dibenzo[de,uv]pentaphene

347

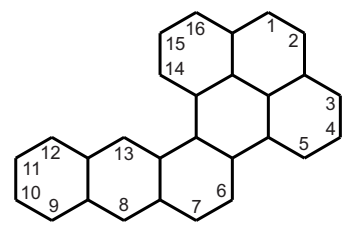

Dibenzo[a,rst]pentaphene<smiles>C1CCC2C(C1)CC1CCC3CC4CCCCC4C4C(CCC2C14)C3</smiles>

348
120835-51-0 $\quad \mathrm{C}_{28} \mathrm{H}_{16}$

352

1.532

16.27
10.63

5.126

1.515
5.69
0.35
6.132



1.530
15.97
10.44
5.407

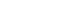


349

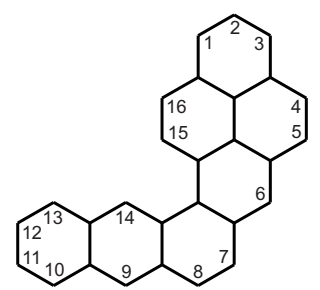

Naphtho[2,1,8-uva]pentaphene

Dibenzo[de,ij]pentaphene

350

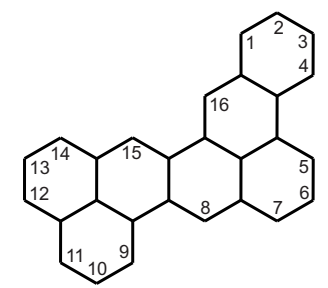

351<smiles>C1CC2CCC3C4CCCC5CCC6CCCC(C7CCC(C1)C2C37)C4C56</smiles>

Naphtho[2,1,8-def]picene

Dibenzo[fg,ij]pentaphene

Dibenzo[b, $n]$ perylene

2,3,10,11-Dibenzoperylene

353

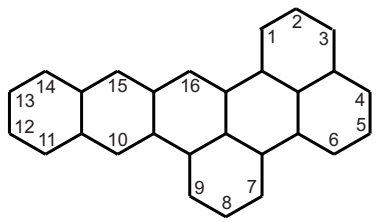

Dibenzo[de,st]pentacene

Naphtho[2,1-b]perylene

Dibenzo[a,pqr]picene

Dibenzo[fg, $q r]$ pentacene

Dibenzo[b,k]perylene

2,3,8,9-Dibenzoperylene

354

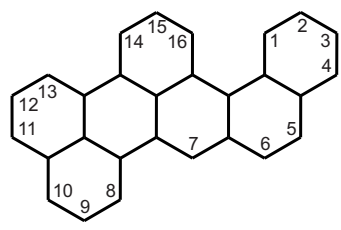

355

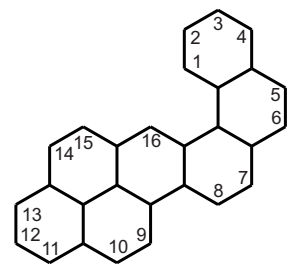

356
121159-18-0 $\quad \mathrm{C}_{28} \mathrm{H}_{16}$

352

1.535

16.26

10.59
5.491

120835-46-3 $\quad \mathrm{C}_{28} \mathrm{H}_{16}$

352

1.538

16.18

10.52
3.896

120835-39-4 $\quad \mathrm{C}_{28} \mathrm{H}_{16}$

352

1.541

16.07
10.42

5.354

197-69-3

$\mathrm{C}_{28} \mathrm{H}_{16}$

352

1.542

16.01

10.38

$14147-38-7$

$\mathrm{C}_{28} \mathrm{H}_{16}$

352

1.557

16.85

4.843<smiles>C1CCC2C(C1)CC1C3CCCC4C5CCCCC5C5CCCC(C21)C5C43</smiles>

197-74-0

$\mathrm{C}_{28} \mathrm{H}_{16}$

352
1.583

16.24

10.26 5.420

\subsection{1 \\ 16.23 \\ 5.430}

$\begin{array}{lll} & 352 & 1.612 \\ & & 16.76 \\ & 10.40 \\ & 3.904\end{array}$


<smiles>C1CCC2CC3CC4C(CC5CCCC6CCCC4C65)C3CCCC2C1</smiles>

120835-53-2 $\quad \mathrm{C}_{28} \mathrm{H}_{16}$

352

1.643

16.91

10.30

3.888

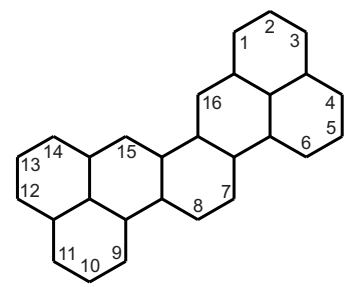

Dibenzo[de,k]pentaphene

358

Dibenzo[de,k $]$ pentaphene

Benzo[mno]naphtho[1,2-c]chrysene

359<smiles>C1CC2CCC3CC4CCC5C6CCCCC6CCC5C4C3CCC2C1</smiles><smiles></smiles>

Benzo[a]naphtho[8,1,2-Imn]-

naphthacene

190-01-2

$\mathrm{C}_{28} \mathrm{H}_{16}$

352

1.765

16.98
9.619

3.955

361

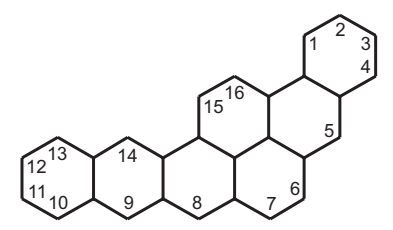

Benzo[vwx]hexaphene

2828-72-0

$\mathrm{C}_{28} \mathrm{H}_{16}$

352

1.857

18.29

3.892

362<smiles>C1CCC2C(C1)CCC1CC3C(CC4CCC5CCCC6CCC(C4)C3C56)CC12</smiles>

Benzo[a]naphtho[2,1,8-hij]naphthacene

190-05-6

$\mathrm{C}_{28} \mathrm{H}_{16}$

352

1.897

18.19
9.589

9.589
3.902

363

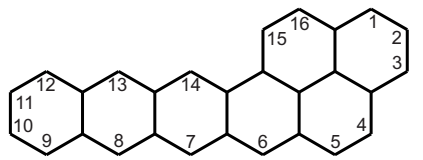

Naphtho[2,1,8-uva]pentacene

364<smiles></smiles>

Anthra[2,1,9-qra]naphthacene

365<smiles>C1=CCC2CC3C(CCC2C1)CC1CCC2CCCC4CCC(C3C4)C21</smiles>

Naphtho[8,1,2-cde]pentaphene

196-45-2

$\mathrm{C}_{28} \mathrm{H}_{16}$

352

1.918

18.56

9.690

$\begin{array}{llll}189-52-6 & \mathrm{C}_{28} \mathrm{H}_{16} & 352 & 1.937 \\ & & & 18.56 \\ & & 9.583 \\ & & & \\ & & & \\ & & & \\ 120835-50-9 & \mathrm{C}_{28} \mathrm{H}_{16} & 352 & 1.960 \\ & & & 18.20 \\ & & & 9.286 \\ & & & 3.959\end{array}$


366

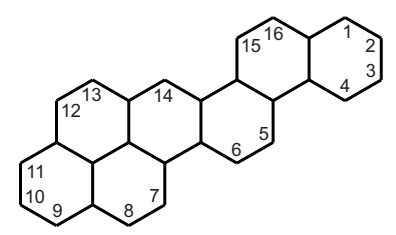

Dibenzo[c,pqr]picene

120835-44-1 $\quad \mathrm{C}_{28} \mathrm{H}_{16}$

352

1.964

18.24<smiles></smiles>

Dibenzo[c,rst]pentaphene

120835-52-1 $\quad \mathrm{C}_{28} \mathrm{H}_{16}$

352

1.965

18.25

9.284
3.911<smiles></smiles>

Dibenzo[b,tuv]picene

Benzo[a]naphtho[2,1-h]pyrene

189-18-4

$\mathrm{C}_{28} \mathrm{H}_{16}$

352

1.966

18.25

9.282

3.921

369<smiles>C1=CC2CC3C(CCC2CC2CCCCC2C1)CC1C2CC4CCCCC4CC2CCCC31</smiles>

13H-Dibenzo[a,de]naphth[2,3-h]anthracene

$\mathrm{C}_{29} \mathrm{H}_{18}$

366

1.672

17.07

10.21

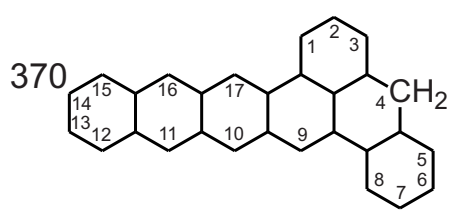

4H-Dibenzo[a,de]pentacene

$\mathrm{C}_{29} \mathrm{H}_{18}$

366

1.674

18.49

11.05
4.982 
371

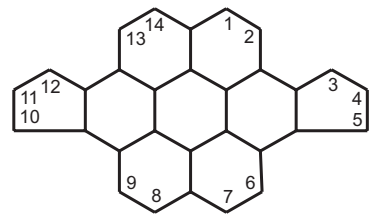

Dicyclopenta[a,j]coronene

Dibenz [e,ghi]indeno-

[1,2,3,4-pqra]perylene

Dibenzo[ $m n, q r]$ fluoreno-

[2,1,9,8,7-defghi]naphthacene

Dibenzo[bc,ef]coronene

2,3,4,5-Dibenzocoronene

Anthrodianthrene

vic-diperi-Dibenzocoronen

375<smiles></smiles>

376

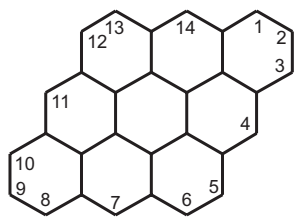

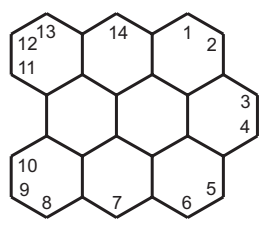

Naphtho[8,1,2-abc]coronene Naphtho[5,4,3-abc]coronene

Dibenzo[bc,k]coronene

Dibenzo[ijk,tuv]peropyrene

2,3,8,9-Dibenzocoronene

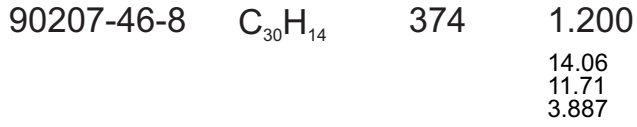

75449-96-6 $\quad \mathrm{C}_{30} \mathrm{H}_{14}$

$374 \quad 1.117$

13.92

12.47

3.902

76759-99-4 $\quad \mathrm{C}_{30} \mathrm{H}_{14} \quad 374 \quad 1.250$

14.42

31.53

6596-38-9

$\mathrm{C}_{30} \mathrm{H}_{14}$

374

1.212

14.22

3.888

190-55-6

$\mathrm{C}_{30} \mathrm{H}_{14}$

374

1.351

15.93

11.79
3.888 
377

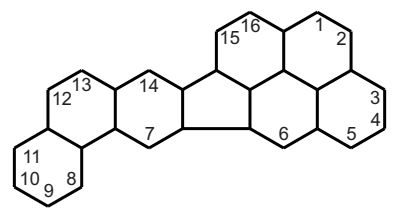

Naphth[1',2':5,6]indeno[1,2,3-cd]pyrene

Naphth[2', 1':4,5]indeno[1,2,3-cd]pyrene

Tribenzo[a,e,ghi]perylene

Benzo[a]naphtho[2,1,8-cde]perylene

Dibenzo[c,hi]naphtho[3,2,1,8-mnop]chrysene

120835-72-5 $\quad \mathrm{C}_{30} \mathrm{H}_{16}$

376

1.142

14.32

12.54<smiles>CC1CCC2CC3CCCCC3CC2C1</smiles><smiles></smiles>

382

Benzo[b]naphtho[1,2,3,4-pqr]perylene

Tribenzo[b,e,ghi]perylene

53156-62-0

$\mathrm{C}_{30} \mathrm{H}_{16}$

376

1.163

13.74

11.82 5.600

383

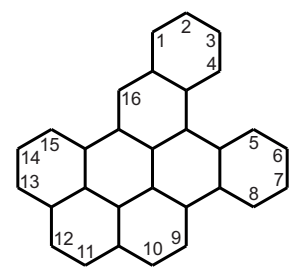

120835-62-3 $\quad \mathrm{C}_{30} \mathrm{H}_{16}$ 376 
384

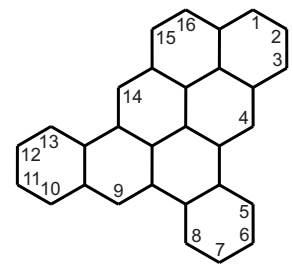

\section{Benzo[ $h]$ naphtho[7,8,1,2,3-pqrst]-} pentaphene

120835-69-0

$\mathrm{C}_{30} \mathrm{H}_{16}$

376

1.226

15.99

13.04
4.267

385<smiles>C1CCC2C(C1)CC1CCC3CC4C5CCCCC5C5CC6CCCCC6C4C5C3CC12</smiles>

Phenanthro[9,10,1,2,3-pqrst]-

120835-79-2 $\quad \mathrm{C}_{30} \mathrm{H}_{16}$

$376 \quad 1.230$

pentaphene

16.02

13.03
4.201

386

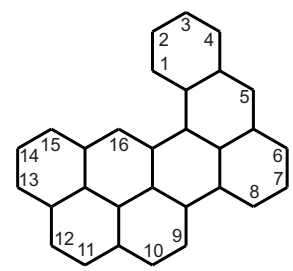

Benzo[a]naphtho[2,1,8-Imn]perylene

120835-71-4

$\mathrm{C}_{30} \mathrm{H}_{16}$

376

1.233

14.40

11.68

387

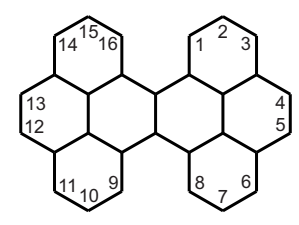

Tetrabenzo[de,hi,mn,qr]naphthacene

385-13-7

$\mathrm{C}_{30} \mathrm{H}_{16}$

376

1.277

14.27

11.18
6.050

388

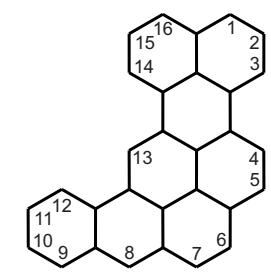

Anthra[9,1,2-bcd]perylene

120864-21-3 $\quad \mathrm{C}_{30} \mathrm{H}_{16}$

376

1.340

15.64

15.64
3.902

389

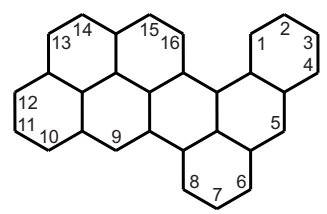

Benzo[a]naphtho[8,1,2-k/m]perylene

120835-73-6

$\mathrm{C}_{30} \mathrm{H}_{16}$

376

1.348

15.37

11.40

390

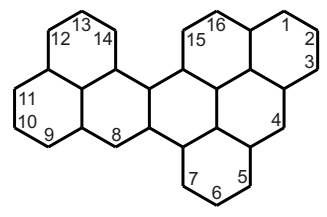

Dibenzo[de,mn]naphtho[2,1,8-qra]-

$120835-68-9$

$\mathrm{C}_{30} \mathrm{H}_{16}$

376

1.352 naphthacene 


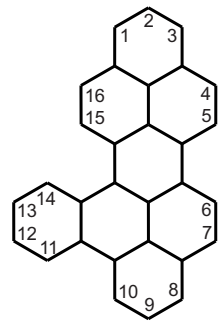

Tribenzo[a,cd,Im]perylene

$92411-20-6$

$\mathrm{C}_{30} \mathrm{H}_{16}$

376

1.354

15.79

5.663

Phenanthro[1,2,3,4-ghi]perylene

$120835-58-7$

$\mathrm{C}_{30} \mathrm{H}_{16}$

376

1.355

15.90

11.74
5.375

393

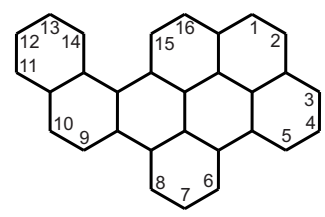

Benzo[pqr]naphtho[1,2-b]perylene

120835-57-6

$\mathrm{C}_{30} \mathrm{H}_{16}$

376

1.357

15.93
11.74

5.574<smiles>C1CC2CCCC3C4C(CCC(C1)C24)C1CCCC2CCCC4C2C1C43</smiles>

394

Benzo[Im]naphtho[1,8-ab]perylene

Benzo[rst]naphtho[2,1,8-fgh]pentaphene

Benzo[a]naphtho[1,2,3,4-ghi]perylene

$\begin{array}{llll}\text { 97938-05-1 } \quad \mathrm{C}_{30} \mathrm{H}_{16} \quad 376 \quad 1.363 \\ & & & 15.79 \\ & & 11.59 \\ & 5.573\end{array}$

120835-64-5 $\quad \mathrm{C}_{30} \mathrm{H}_{16}$ $376 \quad 1.363$

16.03 11.76
4.469 396

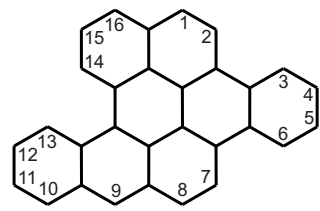

120835-76-9 $\quad \mathrm{C}_{30} \mathrm{H}_{16}$ 
397

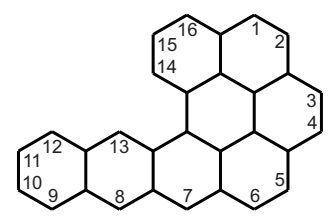

398

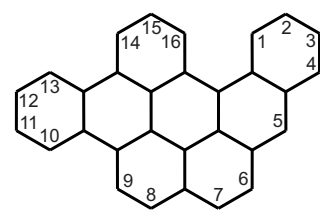

399

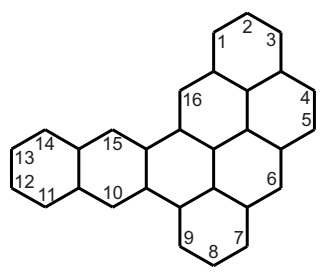

400<smiles></smiles>

401<smiles>C1CCC2C(C1)CC1C3CCCCC3CC3C4CCCCC4C4CCCCC5CCCC2C5C1C43</smiles>

402

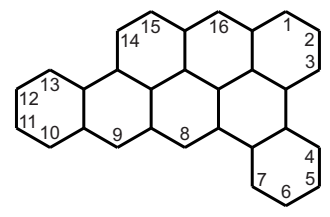

403

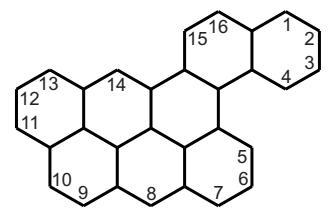

Benzo[uv]naphtho[2,1,8,7-defg]pentaphene

Tribenzo[a,ghi,k]perylene

Anthra[2,1,9,8-defgh]pentaphene

Benzo[ghi]naphtho[2,1-a]perylene

Dibenzo[ $b, q r]$ naphtho[3,2,1,8-defg]chrysene

Anthra[8,9,1,2-cdefg]benzo[a]naphthacene

Benzo[de]naphtho[8,1,2,3-stuv]picene
120835-86-1 $\quad \mathrm{C}_{30} \mathrm{H}_{16}$

376

1.365

15.95

11.68

5.330

$\begin{array}{llll}120835-75-8 \quad \mathrm{C}_{30} \mathrm{H}_{16} \quad 376 \quad 1.367 \\ & & 15.91 \\ & & 11.64 \\ & 5.592\end{array}$

120835-77-0 $\quad \mathrm{C}_{30} \mathrm{H}_{16} \quad 376 \quad 1.371$

16.05

11.71

4.362

$\begin{array}{llll}120835-59-8 \quad \mathrm{C}_{30} \mathrm{H}_{16} \quad 376 \quad 1.371 \\ & & & 16.00 \\ & & 11.67 \\ & 5.221\end{array}$

120835-61-2 $\quad \mathrm{C}_{30} \mathrm{H}_{16} \quad 376 \quad 1.381$

16.09

11.65
3.904

120835-82-7 $\quad \mathrm{C}_{30} \mathrm{H}_{16} \quad 376 \quad 1.416$

16.22

11.46
3.900

120835-74-7 $\quad \mathrm{C}_{30} \mathrm{H}_{16} \quad 376 \quad 1.445$ 
<smiles></smiles>

Benzo[ghi]naphtho[2,1-b]perylene

120835-63-4

$\mathrm{C}_{30} \mathrm{H}_{16}$

376

1.470

16.30

11.09
5.224

405<smiles>C1CCC2CC3C(CCC2C1)CC1CCCC2CCC4CCC5CCC(C1)C2C5C43</smiles>

Benzo[uv]naphtho[2,1,8,7-defg]pentacene

5869-31-8

$\mathrm{C}_{30} \mathrm{H}_{16}$

376

1.476

16.87
11.43

3.903

406

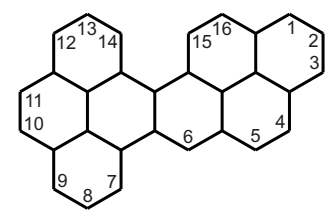

Benzo[pqr]naphtho[2,1,8-def]picene

100684-90-0

$\mathrm{C}_{30} \mathrm{H}_{16}$

376

1.477

16.20

10.97
5.659

407<smiles>C1CCC2C(C1)CC1CCC3CC4CCCC5CCCC6CC(C1)C2C3C4C56</smiles>

Benzo[a]naphtho[7,8,1,2,3-pqrst]pentaphene

120835-78-1

$\mathrm{C}_{30} \mathrm{H}_{16}$

376

1.484

16.25

10.96
5.418

408

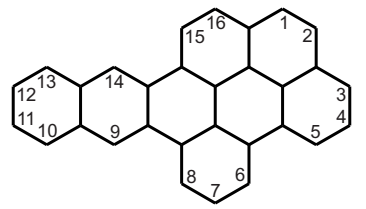

Benzo[st]naphtho[2,1,8,7-defg]pentacene

14258-76-5

$\mathrm{C}_{30} \mathrm{H}_{16}$

376

1.494

16.76
11.22

3.902

409<smiles>C1CCC2C(C1)CC1C3CCC4CCC5C6CCCCC6C6CCCC1C6C5C4C23</smiles>

Benzo[qr]naphtho[2,1,8,7-fghi]pentacene

190-87-4

$\mathrm{C}_{30} \mathrm{H}_{16}$

376

1.498

16.76

11.19

410<smiles>C1=CC2CCC3CCC4C5CC(CCCC5C5CCCC(C1)C2C35)C1C2CCCCC2CCC41</smiles>

Benzo[pqr]naphtho[2,1-b]perylene

$120835-56-5$

$\mathrm{C}_{30} \mathrm{H}_{16}$

376

1.524

15.95

10.47

411<smiles></smiles>

Tribenzo[b,n,pqr]perylene

$190-81-8$

$\mathrm{C}_{30} \mathrm{H}_{16}$

376

1.539 


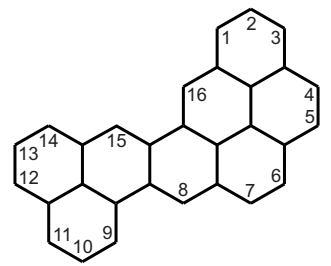

Benzo[k/naphtho[2,1,8,7-defg]-

Benzo[ij]naphtho[2,1,8,7-defg]pentaphene

Tribenzo[de,ij,rst]pentaphene

Benzo[de]naphtho[2,1,8,7-qrst]pentacene

Benzo[qr]naphtho[2,1,8,7-defg]pentacene

Naphtho[3,2,1,8,7-vwxyz]hexaphene

417

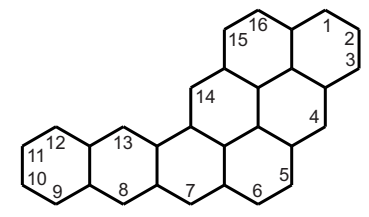

418<smiles></smiles>

419

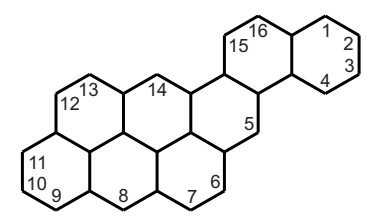

Pyranthrene

Benzo[c]naphtho[7,8,1,2,3-pqrst]pentaphene

120835-70-3 $\quad \mathrm{C}_{30} \mathrm{H}_{16}$

376

1.540

16.10

10.45

$\begin{array}{llll}\text { 105786-27-4 } & \mathrm{C}_{30} \mathrm{H}_{16} \quad 376 & 1.542 \\ & & & 16.06 \\ & & 10.42 \\ & 5.149\end{array}$

120835-65-6 $\quad \mathrm{C}_{30} \mathrm{H}_{16}$

376

1.546

16.08

10.40

5.151

\begin{tabular}{|c|c|c|}
\hline 120835-83-8 & $\mathrm{C}_{30} \mathrm{H}_{16}$ & 376 \\
\hline $120000-00-0$ & $\cup_{30} \Pi_{16}$ & 310 \\
\hline
\end{tabular}

120835-67-8 $\quad \mathrm{C}_{30} \mathrm{H}_{16}$

376

1.609

16.74

10.40
3.907

191-13-9

$\mathrm{C}_{30} \mathrm{H}_{16}$

376

1.731

18.22

10.53
3.939

120835-80-5 $\quad \mathrm{C}_{30} \mathrm{H}_{16}$

376

1.735 


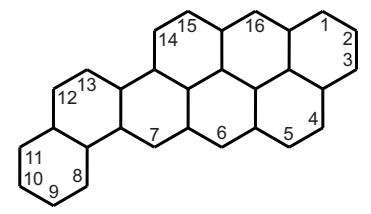

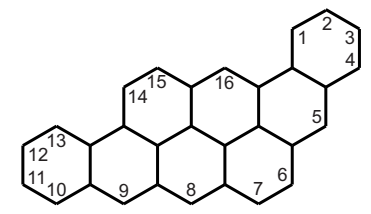

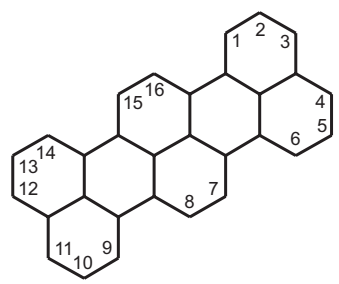

Tribenzo[de,kl,rst]pentaphene Terrylene

$\mathrm{C}_{30} \mathrm{H}_{16}$ 376

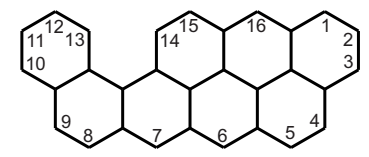

Anthra[8,9,1,2-Imnop]benzo[a]naphthacene

120835-87-2 $\quad \mathrm{C}_{30} \mathrm{H}_{16}$

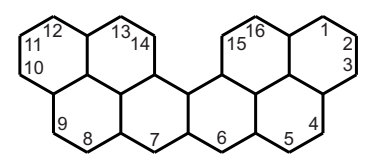

Dinaphtho[8,1,2-Imn:2', 1', 8'-qra]naphthacene

58052-99-6

$\mathrm{C}_{30} \mathrm{H}_{16}$

376

1.784

16.79
9.411

5.326

425

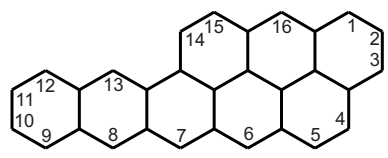

Anthra[2,1,9,8-stuva]pentacene

120835-88-3 $\quad \mathrm{C}_{30} \mathrm{H}_{16}$

376

1.850

17.94

9.695 3.886

426<smiles></smiles>

Naphthaceno[2,1,12,11-opqra]naphthacene

$188-42-1$

$\mathrm{C}_{30} \mathrm{H}_{16}$ 376

1.860

17.93 9.640

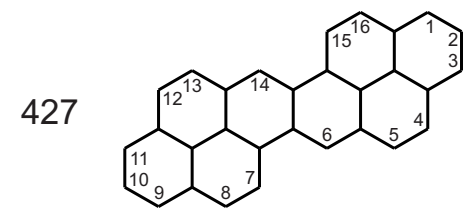

Benzo[pqr]naphtho[8,1,2-cde]picene

111728-58-6 $\quad \mathrm{C}_{30} \mathrm{H}_{16}$ 376<smiles>[Mg][Ge]</smiles>

Benzo[rst]naphtho[8,1,2-cde]-

52879-10-4

$\mathrm{C}_{30} \mathrm{H}_{16}$

376 
<smiles>C1CCC2CC3(CCC2C1)C1CCCCC1CCC1C2CCCCC2CCC13</smiles><smiles>C1=CC2CCC3CCC4CC5CCCCC5CC4C3C2C2CCCCCC12</smiles>

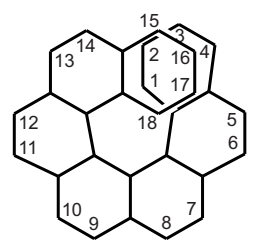<smiles>C1=CC2CC3CC4CCCCC4CC3C3CC4CCCCC4CC3C2CC2CCCCC2C1</smiles>

\section{Trinaphthylene}

195-62-3

$\mathrm{C}_{30} \mathrm{H}_{18}$

378

1.129

Naphtho[2,3- $h]$ pentaphene

Dinaphth[2,3-a,2',3'-c]anthracene

433<smiles>C1CCC2CC3CC4C(CCC3CC2C1)CCC1CCC2CCC3CCCCC3C2CC14</smiles>

Anthra[1,2-c]benz[j]anthracene

115747-59-6 $\quad \mathrm{C}_{30} \mathrm{H}_{18}$

378<smiles>C1CCC2CC3C(CCC2C1)C1CC2CCCCC2CC1C1CC2CCCCC2CC31</smiles><smiles></smiles> 
436<smiles>C1CCC2C(C1)CC1CC3C(CC12)C1CC2CCCCC2C1CC1CCC2CCCCC2C13</smiles>

Phenanthro[3,2-g]chrysene

Dinaphth[1,2-a:2',1'-j]anthracene

Dibenzo[a,h]pentaphene

438<smiles>C1=CC2C(CCC3CCCCC32)CCC2CCC3CCC4CCCC5CCC(C1)C2C4C53</smiles><smiles>C1CCC2CC3C(CC2C1)CC1CCCCC1C1CC2CCCCC2CC31</smiles>

439<smiles>C1CCC2CC3CC4CCCCC4C4CC5CCCCC5CC4C3CCC2C1</smiles>

Benzo[b]naphtho[2,3-g]chrysene

Benzo[b]naphtho[2,1-p]chrysene

Phenanthro[9,10-b]triphenylene

Tetrabenz $[a, c, h]$,$] anthracene$

Tribenzo[b,g,p]chrysene

440<smiles>C1CCC2CC3C4CCCCC4C4CC5CCCCC5CC4C3CCC2C1</smiles>

441<smiles>C1CCC2C(C1)C1CCCCC1C1CC3C4CCCCC4C4CCCCC4C3CC21</smiles>

442
115747-41-6 $\quad \mathrm{C}_{30} \mathrm{H}_{18}$

378

1.235

15.81

5.712

68171-26-6

$\mathrm{C}_{30} \mathrm{H}_{18}$

378

1.237

14.40

5.502

115747-38-1 $\quad \mathrm{C}_{30} \mathrm{H}_{18}$

378

1.241

16.28
13.12
5.32

5.032<smiles>C1CCC2C(C1)C1CC(C3CCCCC23)C2C3CCCCC3C2C2CCCCC12</smiles>

115747-65-4 $\quad \mathrm{C}_{30} \mathrm{H}_{18}$

378

1.241

16.04
12.92

5.419

$\begin{array}{llll}115747-67-6 \quad \mathrm{C}_{30} \mathrm{H}_{18} \quad 378 \quad & 1.251 \\ & & 16.17 \\ & & 12.93 \\ & 5.772\end{array}$

215-11-2

$\mathrm{C}_{30} \mathrm{H}_{18}$

378

1.252

14.21
11.36

5.953

$\begin{array}{llll}115747-77-8 \quad \mathrm{C}_{30} \mathrm{H}_{18} \quad 378 & 1.255 \\ & & & 15.45 \\ & 12.31 \\ & 6.694\end{array}$ 
443<smiles>C1CCC2C(C1)CCC1C2C2CCCCC2C2C3CCCCC3C3CCCCC3C12</smiles>

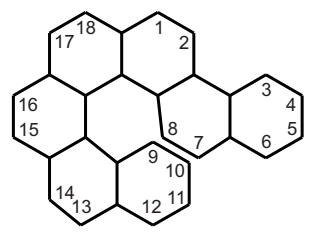<smiles>C1CCC2C(C1)CCC1C2CC2C3CCC4CCCCC4C3C2CC2C3CCCCC3CCC21</smiles><smiles>C1CCC2C(C1)CCC1C2CCC2C3C4CCCCC4CCC3C3CCCCC3C12</smiles>

Dibenzo[a,f]picene

115747-36-9 $\quad \mathrm{C}_{30} \mathrm{H}_{18}$ 378

1.314<smiles>C1CCC2CC3CC4C(CCC3CC2C1)CCC1CC2CC3CCCCC3CC2CC14</smiles><smiles>C1=CC2CCCC3CCCCC(C4CC5C(CCC6CC7CCCCC7CC65)CC14)C23</smiles>

Naphtho[1,2-a]pentaphene

115737-85-8 $\quad \mathrm{C}_{30} \mathrm{H}_{18}$ 378<smiles>C1CCC2C(C1)CC1C2CCC2CC3CCCCC3C2C2CCCCC12</smiles> 
450<smiles>C1CCC2CC3C(CCC2C1)C1CCC2CCC4CCCCC4C2CC13</smiles>

Phenanthro[2,3-c]chrysene

$$
\text { 115747-61-0 } \quad \mathrm{C}_{30} \mathrm{H}_{18}
$$

378

1.385

16.08
11.61 5.742

451<smiles>C1CCC2CC3C(CCC2C1)CC1CCC2CC4CCCCC4CC2CC13</smiles>

Dibenzo[a,o]pentaphene

115747-60-9 $\quad \mathrm{C}_{30} \mathrm{H}_{18}$

378

1.405

16.46
11.72

3.888

452<smiles>C1CCC2C(C1)CCC1C2CCC2C3CCCCC3C2C2CCC3CCCCC3C12</smiles>

453<smiles>C1CCC2C(C1)CCC1CCC3CC4C5CCCCC5C5CCCCC5C4CC3C12</smiles>

Phenanthro[3,4-b]triphenylene

Dibenzo[a,]picene

115747-54-1 $\quad \mathrm{C}_{30} \mathrm{H}_{18}$

378

1.423

16.11

11.32
5.262

Naphtho[1,2-f]picene

115747-49-4 $\quad \mathrm{C}_{30} \mathrm{H}_{18}$

112498-95-0 $\quad \mathrm{C}_{30} \mathrm{H}_{18}$

378

1.409

15.62

5.474

454<smiles>CC1CC2CCC3C(CCC4CCC5CCCCC5C43)C2C2CCCCC12</smiles>

455<smiles>C1CCC2C(C1)CCC1C2CCC2CC3CCCCC3C2C2CCCCC12</smiles>

Tribenzo[ $[b, g$,$] chrysene$

115747-47-2 $\quad \mathrm{C}_{30} \mathrm{H}_{18}$ 378

1.444

16.71
11.58 5.992<smiles>C1CCC2C(C1)CCC1CCC3CC4CCC5CCCCC5C4CC3CC12</smiles> 
457<smiles>C1CCC2CC3C(CCC4CCC5C6CCC7CCCCC7C6CCC5C43)CC2C1</smiles>

458<smiles>C1CCC2CC3C(CCC4CC5CCC6CC7CCCCC7CC6C5CC43)CC2C1</smiles>

Naphtho[2,3-a]pentaphene

Phenanthro[3,4-b]chrysene

459<smiles>C1=CC2C3CCCCC3C3CCC4CC5CCC6CCC7CCCCC7C6CC5C4CC3C2CC1</smiles>

460<smiles>C1CCC2C(C1)CCC1CC3CCC4C5CCCCC5C5CCCCC5C4CC3C12</smiles>

Benzo[g]naphtho[2,1-b]chrysene

Dibenzo[f,jpicene

461<smiles>C1CCC2C(C1)CCC1C3CCCCC3C3CCCCC3C1C1CCCCC21</smiles>

462<smiles>C1CCC2CC3C(CC2C1)CC1CC2CCCCC2C2CCCCC2CC13</smiles>

463<smiles>C1=CCC2C(C1)CCC1CCC3C4CCC5CCCCC5C4C3CCC12</smiles>

Dibenzo[f,]picene

Dibenzo[c,h]pentaphene

Dibenzo[a,o]picene
115747-58-5

$\mathrm{C}_{30} \mathrm{H}_{18}$

378

1.500

17.11

5.475

115747-73-4 $\quad \mathrm{C}_{30} \mathrm{H}_{18}$

378

1.503

17.34

11.54
4.027

115747-84-7 $\quad \mathrm{C}_{30} \mathrm{H}_{18}$

378

1.503

16.93

11.26
5.177

Benzo[g]naphtho[2,1-b]chrysene

115747-57-4

$\mathrm{C}_{30} \mathrm{H}_{18}$

378

1.515

16.37

10.80
6.072

188-67-0

$\mathrm{C}_{30} \mathrm{H}_{18}$

378

1.516

16.23

10.70

115747-39-2 $\quad \mathrm{C}_{30} \mathrm{H}_{18}$

378

1.545

18.12
11.73
5.068

115747-93-8 $\quad \mathrm{C}_{30} \mathrm{H}_{1}$

378

1.558 
464<smiles>C1CCC2C(C1)CCC1CC3CC4C5CCCCC5C5CCCCC5C4CC3CC12</smiles>

Tribenzo[a,c,j]naphthacene

215-96-3

$\mathrm{C}_{30} \mathrm{H}_{18}$

378

1.582

17.96

11.36
4.768

465<smiles>C1CCC2CC3CCC4CC5C6CCCCC6C6CCCCC6C5CC4C3CCC2C1</smiles>

Dibenzo[a,c]pentaphene

Dibenzo[ $[b, f]$ picene

466<smiles>C1CCC2C(C1)CC1C2CC2CCCCC2C2C3CCCCC3C3C4CCCCC4CCC3C12</smiles>

467<smiles>C1=CC2C(CC1)C1CCCCC1C1CC3CC4CC5CCCCC5CC4CC3CC21</smiles>

Dibenzo[a,c]pentacene

Benzo[a]naphtho[1,2-c]naphthacene

Benzo[p]naphtho[2,1-b]chrysene

469<smiles>C1CCC2CC3C(CC2C1)CC1C(CC2CCCCC23)CC2CC3CCCCC3CC21</smiles><smiles>C1CCC2C(C1)CC1C2CC2CC3CCCCC3C2C2C3CCCCC3CCC12</smiles>

470<smiles>C1CCC2CC3CC4C5CCCCC5CCC4C3C3CCCCC3CC2C1</smiles> 
471<smiles>C1CCC2CC3CCC4CC5CC6CCCCC6CC5CC4C3CCC2C1</smiles>

Benzo[a]hexaphene

472<smiles></smiles>

Phenanthro[1,2-a]naphthacene

473<smiles>C1CCC2C(C1)CC1C3CCC4C5CCCCC5C4CCC3CC1C1CCCCC21</smiles>

474<smiles>C1CCC2CC3C(CCC4C3CCC3CCCCC3C3CC5CCCCC5C34)CC2C1</smiles>

Phenanthro $[9,10-b]$ chrysene

Phenanthro[1,2- $b]$ triphenylene

Tribenzo $[b, g, k]$ chrysene

475<smiles>C1=CC2C(CC1)CCC1CCC3CC4CC5C(CCC6CCCCC65)CCC4C3CCC12</smiles>

Dinaphth[1,2-a:1',2'-h]anthracene

476<smiles>C1CCC2CC3CC4CC5CC6CCCCC6CC5CCC4CC3CCC2C1</smiles>

Benzo[q]hexaphene

Benzo[c]naphtho[2,3-/]chrysene

477
115747-79-0 $\quad \mathrm{C}_{30} \mathrm{H}_{18}$

378

1.626

18.46

11.35
3.926

115747-63-2 $\quad \mathrm{C}_{30} \mathrm{H}_{18}$

$378 \quad 1.626$

18.23
11.21
5.473

80277-95-8 $\quad \mathrm{C}_{30} \mathrm{H}_{18}$

378

1.638

18.15

11.08
4.979

115747-46-1 $\quad \mathrm{C}_{30} \mathrm{H}_{18}$ 378

1.643

18.39
11.19

5.684

20495-15-2 $\quad \mathrm{C}_{30} \mathrm{H}_{18}$

378

1.660

16.93

10.20

115747-74-5 $\quad \mathrm{C}_{30} \mathrm{H}_{18}$

378

1.702

18.69

10.98
3.898<smiles>C1=CC2CCCCC2C2CCC3CCC4CCC5CCCCC5C4C3C3CC(C1)CC23</smiles>

115747-44-9 $\quad \mathrm{C}_{30} \mathrm{H}_{18}$ 378 
<smiles>C1=CC2C(CCC3CCC4CC5CCCCC5CC4C32)C2CC3CCCCC3CC12</smiles>

479<smiles>C1CCC2CC3C(CCC4C3CCC3C5CCCCC5C5CCCCC5C43)CC2C1</smiles>

Dibenzo[b,j]picene

115747-48-3

$\mathrm{C}_{30} \mathrm{H}_{18}$

378

1.711

18.19
10.63

5.598

480<smiles>C1CCC2CC3CC4CC5C(CCC4CC3CC2C1)CCC1CCCCC15</smiles>

Naphtho[1,2-a]pentacene

115791-74-7 $\quad \mathrm{C}_{30} \mathrm{H}_{18}$

378

1.719

18.40
10.70

10.70
4.979

481

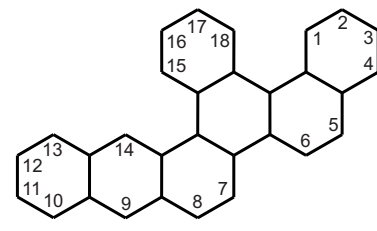

Dibenzo[b,s]picene

115747-88-1 $\quad \mathrm{C}_{30} \mathrm{H}_{18}$

378

1.724

17.82

482<smiles></smiles>

Naphtho[2,1-a]pentaphene

115747-86-9 $\quad \mathrm{C}_{30} \mathrm{H}_{18}$

378

1.728

18.84

10.91
3.976

483

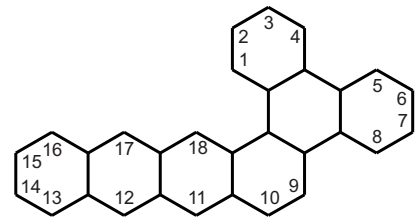

Phenanthro[9,10-a]naphthacene

115791-73-6 $\quad \mathrm{C}_{30} \mathrm{H}_{18}$ 378

1.730

18.14
10.49

5.534

484<smiles>C1CCC2C(C1)CCC1C2CCC2C3CCCCC3C3CCCCC3C12</smiles>

Dibenzo[a,c]picene

115747-70-1 $\quad \mathrm{C}_{30} \mathrm{H}_{18}$

378

1.734 
485<smiles>C1CCC2C(C1)CCC1C2CCC2C3CCCCC3C3C4CCCCC4CCC3C12</smiles>

Dibenzo[c,s]picene

115747-50-7 $\quad \mathrm{C}_{30} \mathrm{H}_{18}$

378

1.746

18.24

10.45

5.227

486<smiles>C1CCC2C(C1)CCC1CC3C(CCC4CC5CCC6CCCCC6C5CC43)CC12</smiles>

Dibenzo[a,m]pentaphene

$$
\text { 115747-83-6 } \quad \mathrm{C}_{30} \mathrm{H}_{18}
$$

378

1.750

18.80

10.75

4.021

487<smiles></smiles>

Naphtho[1,2-b]picene

115747-82-5

$\mathrm{C}_{30} \mathrm{H}_{18}$

378

1.777

18.78
10.57

3.887

488<smiles>C1CCC2CC3CC4CC5CCC6CCCCC6C5CC4CC3CCC2C1</smiles>

Benzo[a]naphtho[1,2-j]naphthacene

$112498-94-9$

$\mathrm{C}_{30} \mathrm{H}_{18}$

378

1.781

18.34

10.30<smiles>C[R16](C)(C)[Na]</smiles>

Phenanthro[4,3- $b]$ chrysene

$42851-11-6$

$\mathrm{C}_{30} \mathrm{H}_{18}$

378

1.797

18.36

10.22
5.326<smiles>CC(C)(C)O[Na]</smiles>

Dibenzo[a,n]picene

$$
\text { 115747-62-1 } \quad \mathrm{C}_{30} \mathrm{H}_{18}
$$

378

1.800

18.41

10.23
5.247<smiles>C1CCC2C(C1)CCC1C2CCC2C3CCC4CCCCC4C3CCC12</smiles>

Naphtho[2,1-a]picene

115747-51-8 $\quad \mathrm{C}_{30} \mathrm{H}_{18}$

378

1.801

18.65

10.35
5.268

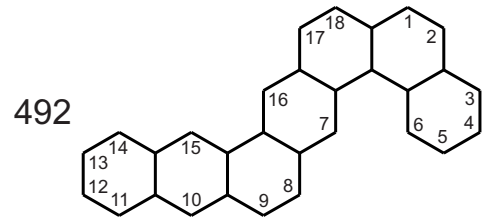

Naphtho[2,1-c]pentaphene 
493<smiles>C1CCC2C(C1)CCC1C2CCC2C1CCC1CCC3CCCCC3C12</smiles>

Naphtho[2,1-c]picene

Benzo[/]naphtho[1,2-b]chrysene

Benzo[c]naphtho[1,2-/]chrysene

495<smiles>C1CCC2C(C1)CCC1CCC3C(CC4CCC5CCCCC5C43)CC12</smiles>

Benzo[ ]naphtho[1,2-b]chrysene

Benzo[/]naphtho[2,1-b]chrysene

Heptaphene

Dibenzo $[b, n]$ pentaphene

Anthra[2,3-a]naphthacene<smiles></smiles>

497<smiles>C1CCC2CC3CC4C(CCC5CC6CC7CCCCC7CC6CC54)CC3CC2C1</smiles>

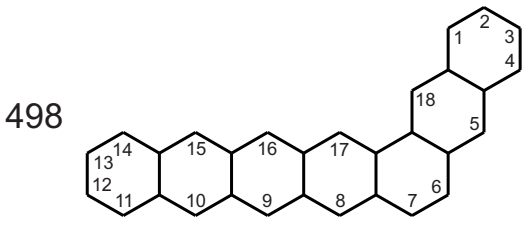

Benzo[p]hexaphene

Benzo[b]naphtho[1,2-/]chrysene

499<smiles>C1CCC2CC3C(CCC4C3CCC3C5CCCCC5CCC34)CC2C1</smiles>

500<smiles>C1CCC2C(C1)CC1CC3CC4CCC5C6CCCCC6CCC5C4CC3CC12</smiles>

Benzo[a]naphtho[1,2-/]naphthacene

$112498-96-1$

115747-43-8 $\quad \mathrm{C}_{30} \mathrm{H}_{18}$

115747-42-7 $\quad \mathrm{C}_{30} \mathrm{H}_{18}$

115747-52-9 $\quad \mathrm{C}_{30} \mathrm{H}_{18}$

378

1.907

18.11

5.198

115747-53-0 $\quad \mathrm{C}_{30} \mathrm{H}_{18}$

222-75-3

$\mathrm{C}_{30} \mathrm{H}_{18}$

378

1.956

20.38
10.42

3.901

$\begin{array}{lll}222-81-1 \quad \mathrm{C}_{30} \mathrm{H}_{18} \quad 378 \quad & 1.991 \\ & & 20.51 \\ & & 10.30 \\ & 3.893\end{array}$

115747-90-5 $\quad \mathrm{C}_{30} \mathrm{H}_{18} \quad 378 \quad 2.019$

19.13
9.475

5.450

501<smiles>C1CCC2CC3CC4CC5CC6C(CCC7CCCCC76)CC5CC4CC3CC2C1</smiles>

Dibenzo[a,n]pentacene

227-07-6

$\mathrm{C}_{30} \mathrm{H}_{18}$

378 
502<smiles>C1CCC2CC3CCC4C5CC6CCCCC6CC5CCC4C3CCC2C1</smiles>

Benzo[b]naphtho[2,1-k]chrysene

115747-91-6 $\quad \mathrm{C}_{30} \mathrm{H}_{18}$

378

2.032

19.38

9.541
4.041

503

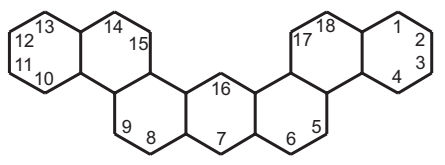

Phenanthro[2,1-b]chrysene

504<smiles>C1CCC2CC3CC4C(CC3CC2C1)CC1CC2C(CCC3CCCCC32)CCC14</smiles>

Phenanthro[3,4-a]naphthacene

505<smiles>C1CCC2CC3CCC4CC5CC6CCCCC6CC5CC4C3CCC2C1</smiles>

Benzo[o]hexaphene

115747-76-7 $\quad \mathrm{C}_{30} \mathrm{H}_{18}$

378

2.062

20.35

9.869
3.899<smiles>CC1CCC2CC3CC4CCC5CC6C(CCC7CCCCC76)CC5C4CC3CC2CC1</smiles>

Benzo[c]hexaphene

115747-92-7 $\quad \mathrm{C}_{30} \mathrm{H}_{18}$

378

2.069

20.54

9.931

3.938

507<smiles>C1CCC2CC3CC4C(CCC5C4CCC4C6CCCCC6CCC45)CC3CC2C1</smiles>

Naphtho[2,3-b]picene

$115747-75-6$

$\mathrm{C}_{30} \mathrm{H}_{18}$

378

2.178

20.59

4.099<smiles>CC(C)[SnH3]</smiles>

Dibenzo[ $b, n]$ picene

Benzo[b]naphtho[2,3-]]chrysene

213-44-5

$\mathrm{C}_{30} \mathrm{H}_{18}$

378

2.213

20.42

9.225
3.897<smiles>CC(C)(C)O[Na]</smiles>

Dibenzo[a,/]pentacene

227-09-8

$\mathrm{C}_{30} \mathrm{H}_{18}$

378

2.222

20.33
9.150

3.890

510<smiles>C1=CCC2CC3CC4CC5C(CCC6CC7CCCCC7CC65)CC4CCC(CC1)C3C2</smiles>

Naphtho[2,3-c]pentaphene

222-58-2

$\mathrm{C}_{30} \mathrm{H}_{18}$

378 
511<smiles>C1=CC2CCC3CC4CCCCC4CCC3CC2CC1CC1CCC2CCCCC2C1</smiles>

Dibenzo[c,m]pentaphene

512<smiles>CCCCC</smiles>

Naphtho[1,2-c]pentaphene

Benzo[a]hexacene

513<smiles></smiles>

514<smiles>C1CCC2C(C1)CC1CC3CC4CCC5C6CCCCC6CCC5C4CC3CC12</smiles>

515<smiles>C1CCC2C(C1)CCC1C2CCC2C3CCC4CCCCC4C3CCC12</smiles>

Naphtho[2,1-b]picene

Dibenzo[b,m]picene

516<smiles></smiles>

Benzo[a]naphtho[2,1-j]naphthacene Phenanthro[3,2-b]chrysene

112498-97-2 $\quad \mathrm{C}_{30} \mathrm{H}_{18}$ 115747-81-4 $\quad \mathrm{C}_{30} \mathrm{H}_{18}$ 378 2.292 20.38 8.892 $\begin{array}{llll}240-04-0 & \mathrm{C}_{30} \mathrm{H}_{18} \quad 378 \quad \begin{array}{ll}2.264 \\ \end{array} & & 20.92 \\ & & 9.241\end{array}$ 3.888

$\begin{array}{llll}115747-80-3 \quad \mathrm{C}_{30} \mathrm{H}_{18} \quad 378 \quad & 2.226 \\ & & & 20.47 \\ & 9.194 \\ & & 3.976\end{array}$

378 2.265 20.45 9.028
3.949 (3)

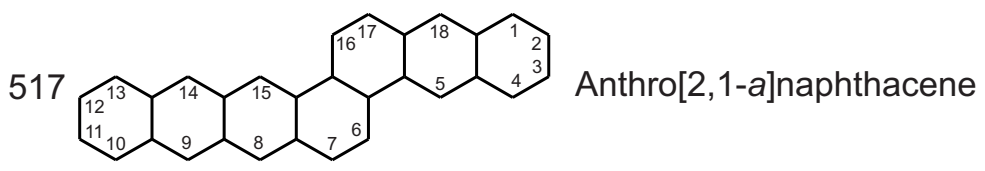

$\begin{array}{llll}214-16-4 & \mathrm{C}_{30} \mathrm{H}_{18} & 378 & 2.331 \\ & & & 21.00 \\ & 9.010 \\ & & 3.902\end{array}$


<smiles>C1CCC2CC3CC4C(CCC5C6CC7CCCCC7CC6CCC54)C3CCC2C1</smiles>

\section{Benzo[b]naphtho[1,2-k]chrysene}

214-15-3

$\mathrm{C}_{30} \mathrm{H}_{18}$

378

2.348 4,5-Benz-10,11-(1',2'-naphtha)chrysene<smiles>C1=CCC2C(C1)CCC1C3CCC4C(CCC5C6CCCCC6CCC45)CC3CCC21</smiles>

519
Phenanthro[1,2-b]chrysene

Dibenzo[c,m]picene
220-78-0

$\mathrm{C}_{30} \mathrm{H}_{18}$

378

2.355

20.55

8.728

520<smiles>C1CCC2C(C1)CCC1C2CCC2C3CCC4CCCCC4C3CCC12</smiles>

521<smiles></smiles>

Heptacene

Ovalene
258-38-8

$\mathrm{C}_{30} \mathrm{H}_{18}$

378

2.886

21.51
7.452

3.891

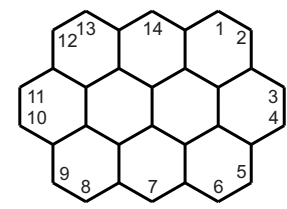

190-26-1
398

1.205 
523

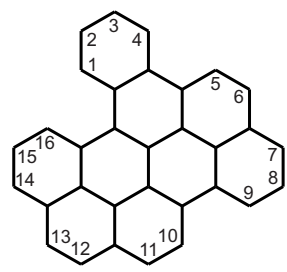

Dibenzo[a,ghi]naphtho[2,1,8-cde]perylene

Dibenzo[a,co]naphtho[8,1,2,3-fghi]perylene

524

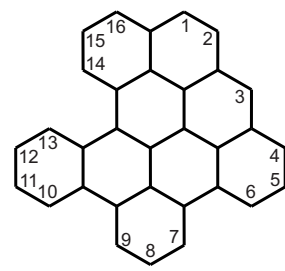

525

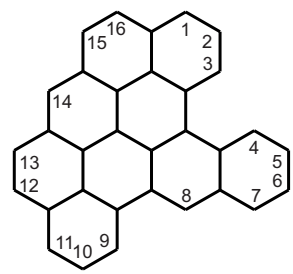

526

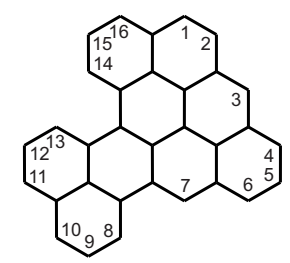

527

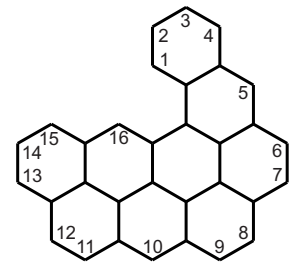

528

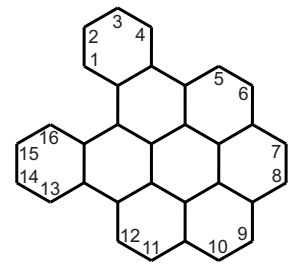

529

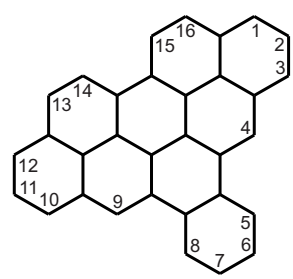

Benzo[ $h]$ phenanthro-

$[2,1,10,9,8,7$-pqrstuv]pentaphene

Benzo[e]phenanthro[2,3,4,5-pqrab]perylene

Dinaphtho[1,8-ab:8', 1',2', 3'-fghi]perylene

Dibenzo[a,ghi]naphtho[2,1,8-Imn]perylene

Dibenzo[a, $d]$ coronene
57789-81-8 $\quad \mathrm{C}_{32} \mathrm{H}_{16}$

400

1.075

13.91

12.94

120835-89-4 $\quad \mathrm{C}_{32} \mathrm{H}_{16}$

400

1.168

13.75
11.77

5.565

120835-96-3 $\quad \mathrm{C}_{32} \mathrm{H}_{16}$

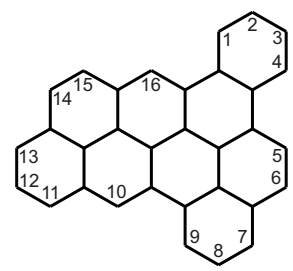


531

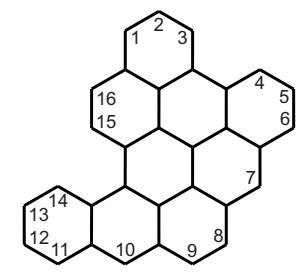

Pyreno[5,4,3,2,1-pqrst]pentaphene

Dinaphtho[2,1,8-fgh:

532

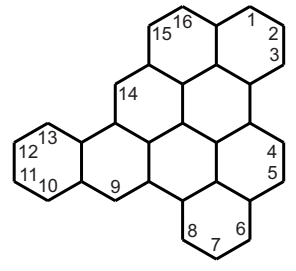

$3^{\prime}, 2^{\prime}, 1^{\prime}, 8^{\prime}, 7^{\prime}-$ rstuv]pentaphene

400

1.229

15.91
12.94

5.167

$120835-93-0$

$\mathrm{C}_{32} \mathrm{H}_{16}$

400

1.233

16.02
12.99

3.894

120835-99-6

$\mathrm{C}_{32} \mathrm{H}_{16}$

400

1.233

$[1,10,9,8$-opqra]perylene

Benzo[3,4]phenanthro[2,1,10,9,8,7-

pqrstuv]pentaphene

120836-12-6 $\quad \mathrm{C}_{32} \mathrm{H}_{16}$

400

1.234

15.83

12.83
5.255<smiles>CCC1CCC2CC3CCCC4CCCC(C)C4C3C2C1C</smiles>

535

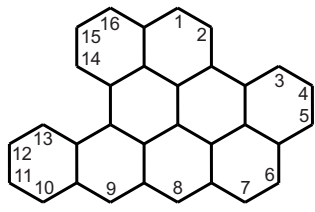

536

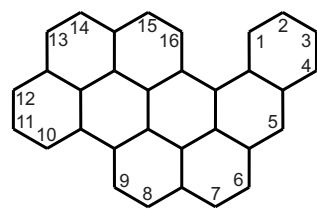

Dibenzo[a,ghi]naphtho-

$[8,1,2-k / m]$ perylene

120836-03-5 $\quad \mathrm{C}_{32} \mathrm{H}_{16}$

400

1.333

15.45

5.298

537

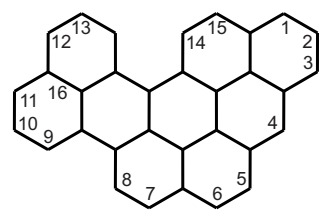

Dibenzo[ghi,Im]naphtho-

$[1,8-a b]$ perylene

120864-23-5 $\quad \mathrm{C}_{32} \mathrm{H}_{16}$

400

1.343

15.44
11.50

5.439

538

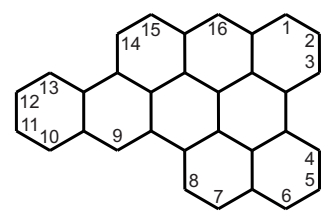

Dibenzo[cd, $k]$ naphtho[3,2,1,8-pqra]perylene

120836-02-4 $\quad \mathrm{C}_{32} \mathrm{H}_{16} \quad 400 \quad 1.349$ 
<smiles></smiles>

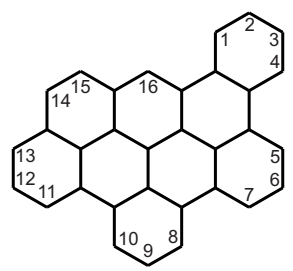

Dibenzo[fg, ij]naphtho[7,8,1,2,3pqrst]pentaphene

120835-91-8 $\quad \mathrm{C}_{32} \mathrm{H}_{16}$

400

1.365

pqrst]pentaphene

Anthra[2,1,9,8-defgh]-

541

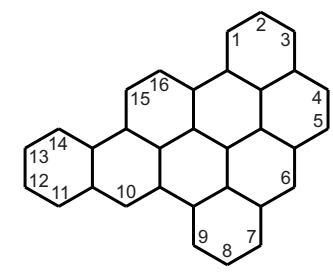

benzo[rst]pentaphene

$\begin{array}{ccc}120836-01-3 \quad \mathrm{C}_{32} \mathrm{H}_{16} \quad 400 \quad & 1.365 \\ & & \\ & & 16.09 \\ & 11.78 \\ & 3.896\end{array}$

542

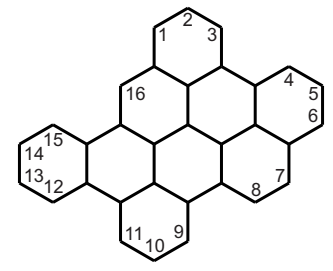

Dibenzo[ $c d, n]$ naphtho-

[3,2,1,8-pqra]perylene

$\begin{array}{llll}109278-09-3 \quad \mathrm{C}_{32} \mathrm{H}_{16} \quad 400 \quad & 1.366 \\ & & 16.02 \\ & 11.73 \\ & 4.318\end{array}$

543

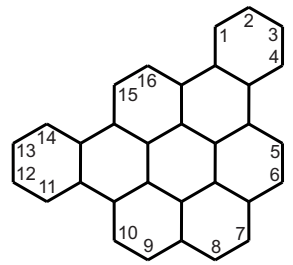

Dibenzo[a,g]coronene

190-66-9

$\mathrm{C}_{32} \mathrm{H}_{16}$

400

1.370

16.10
11.75

3.888

544

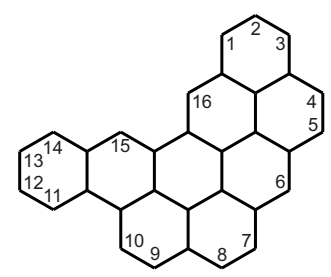

Dibenzo[ghi,n]naphtho[8,1,2-bcd]perylene

120836-05-7 $\quad \mathrm{C}_{32} \mathrm{H}_{16}$

400

1.370

16.07
11.73

3.897

545

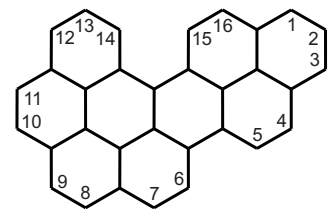

Benzo $[/ m]$ phenanthro[5,4,3abcd]perylene

109278-08-2 $\quad \mathrm{C}_{32} \mathrm{H}_{16}$

400<smiles></smiles> 


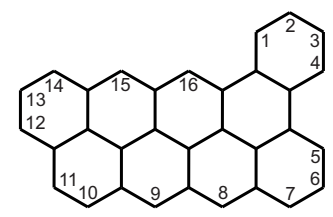

Anthra[3,2,1,9,8-rstuva]benzo-

[ij]pentaphene

Dibenzo[de,ij]naphtho[3,2,1,8,7rstuv]pentaphene

Naphtho[2,3-a]coronene

Anthra[2,1,9,8,7-defghi]benzo-

[st]pentacene

Dibenzo[a,j]coronene

Anthra[2,1,9,8,7-defghi]benzo-

[uv]pentacene

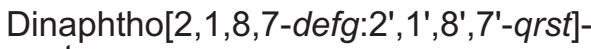
pentacene
120836-16-0

$\mathrm{C}_{32} \mathrm{H}_{16}$

400

1.414

16.20
11.46

3.901

$\begin{array}{llll}120835-92-9 & \mathrm{C}_{32} \mathrm{H}_{16} \quad 400 & 1.414 \\ & & & 15.84 \\ & 11.20 \\ & 4.132\end{array}$

$190-74-9$

$\mathrm{C}_{32} \mathrm{H}_{16}$

400

1.425

16.69

11.71
3.895

120836-08-0

$\mathrm{C}_{32} \mathrm{H}_{16}$

400

1.427

16.75

11.74
3.891

$\begin{array}{llll}190-72-7 \quad & \mathrm{C}_{32} \mathrm{H}_{16} \quad 400 \quad & 1.429 \\ & & 16.73 \\ & & 11.71 \\ & 3.885\end{array}$

120836-13-7 $\quad \mathrm{C}_{32} \mathrm{H}_{16}$

400

1.480

16.88

11.40
3.889

553

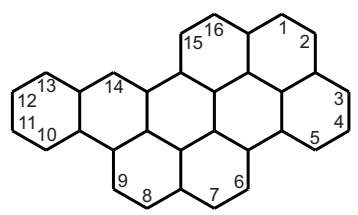

120835-94-1 $\quad \mathrm{C}_{32} \mathrm{H}_{16}$

400

1.497

16.75

11.19
3.887

554

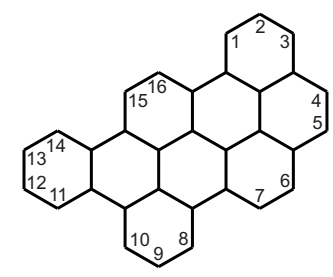

Dibenzo[ij, rst]naphtho[2,1,8,7-defg]pentaphene
$75459-09-5$

$\mathrm{C}_{32} \mathrm{H}_{16}$

400

1.535

16.07

10.47 


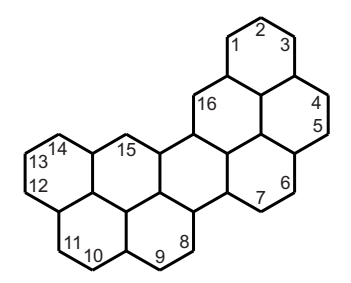

Dinaphtho[2,1,8,7-defg:2', 1', , $\left.8^{\prime}, 7^{\prime}-1 j k \pi\right]-\quad 188-90-9 \quad \mathrm{C}_{32} \mathrm{H}_{16}$

400

1.541 pentaphene

$\begin{array}{rr}188-90-9 \quad \mathrm{C}_{32} \mathrm{H}_{16} \quad 400 \quad & 1.541 \\ & \\ & 16.10 \\ & 10.45 \\ & 3.898\end{array}$

556

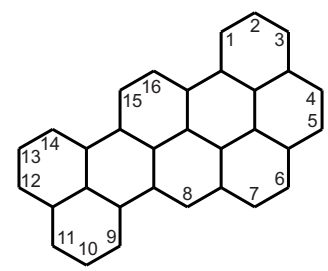

Dibenzo[kl,rst]naphtho[2,1,8,7-defg]pentaphene

120864-22-4

$\mathrm{C}_{32} \mathrm{H}_{16}$

400

1.547

16.12

10.42

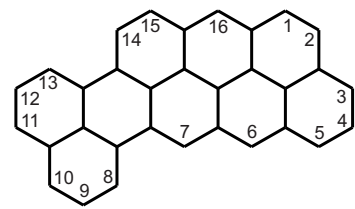

Anthra[2, 1,9,8,7-defghi]benzo[op]pentacene

120864-24-6

$\mathrm{C}_{32} \mathrm{H}_{16}$

400

1.560

16.88

10.82
3.888

558

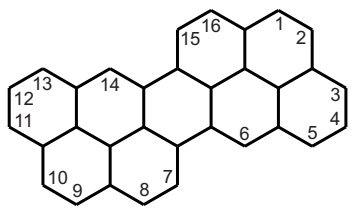

Dinaphtho[2,1,8,7-defg:2',1',8',7'-opqr]pentacene

Dinaphtho[2,1,8-cde, 2', 1', ',-Imn]perylene

2,3,3',2'-Dipyrenylene

188-91-0

$\mathrm{C}_{32} \mathrm{H}_{16}$

400

1.615

16.73

10.36
3.887

559

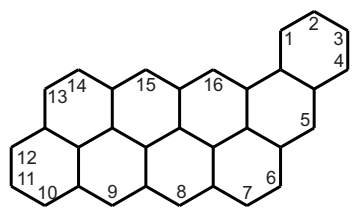

Anthra[7,8,9,1,2,3-rstuvwx]hexaphene

120836-14-8 $\quad \mathrm{C}_{32} \mathrm{H}_{16}$

400

1.650

17.87

10.83

560

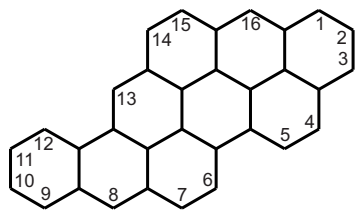

Benzo[def]pyranthrene

72986-34-6

$\mathrm{C}_{32} \mathrm{H}_{16}$

400

1.720

18.15

3.896

561

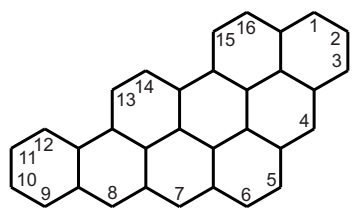

Phenanthro[2,1,10,9,8,7-tuvwxyz]-

120836-17-1 $\quad \mathrm{C}_{32} \mathrm{H}_{16}$

400

1.722 hexaphene

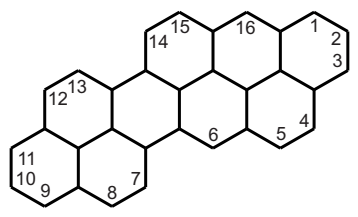

Dinaphtho[8,1,2-cde:7', $8^{\prime}, 1^{\prime}, 2^{\prime}, 3^{\prime}-$ pqrst]-

120835-97-4 $\quad \mathrm{C}_{32} \mathrm{H}_{16}$

400 
563

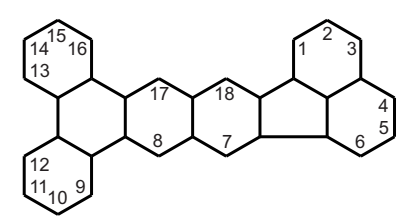

Fluorantheno[8,9-b]triphenylene

Benzo[ $h]$ naphtho[1,2,3,4-rst]pentaphene

Tribenzo[a,f,j]perylene

565<smiles>C1CCC2C(C1)CC1CCCCC1C1C2C2CCCC3CC4CCCCC4C1C32</smiles>

566

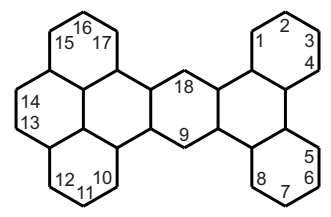

Tetrabenzo[a,c,hi,mn]naphthacene

Anthra[1,2,3,4-rst]pentaphene

567<smiles>C1CCC2CC3C4CCC5CC6CCCCC6C(C5CC2C1)C3C1CCCCC1C4</smiles>

568<smiles>C1CCC2C(C1)CC1CCC3CC4CC5CCC6CCCCC6C5CC4C3CCC12</smiles>

Dibenzo[q, $v w x]$ hexaphene

Dibenzo[fg, st]hexacene

569<smiles>C1CCC2CC3CC4C5CCCC6C(CC7CCCCC76)CC5C4CC3CCCCC2C1</smiles>

Naphtho[2,1,8-yza]hexacene

570

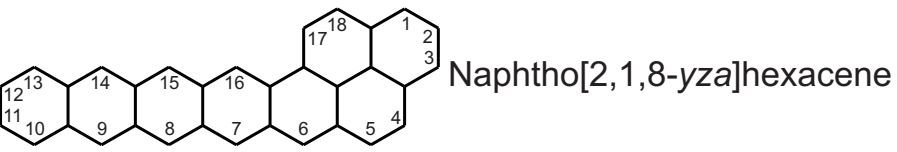

571<smiles>C1CCC2CC3C(CC2C1)CC1CCC2C(CCC13)CC1C3CCCCC3CCC21</smiles>

Benzo[tuv]naphtho[2,1-b]picene

$\begin{array}{cccc}24754-03-8 \quad \mathrm{C}_{32} \mathrm{H}_{18} \quad 402 & 1.518 \\ & & & 17.75 \\ & & 11.69 \\ & & 3.901\end{array}$

31541-02-3 $\quad \mathrm{C}_{32} \mathrm{H}_{18}$

402

1.164

13.66

11.73
5.872

$$
\begin{array}{cccl}
\text { 117440-50-3 } \quad \mathrm{C}_{32} \mathrm{H}_{18} & 402 & 1.248 \\
& & & 15.86 \\
& & 12.71 \\
& 5.916 \\
& & & \\
& & & \\
109587-16-8 \quad \mathrm{C}_{32} \mathrm{H}_{18} & 402 & 1.325 \\
& & & 15.57 \\
& & & \\
& & & 3.758 \\
& & &
\end{array}
$$

$$
\begin{array}{cccc}
31541-07-8 \quad \mathrm{C}_{32} \mathrm{H}_{18} \quad 402 & 1.372 \\
& & & 15.97 \\
& & 11.65 \\
& 4.716
\end{array}
$$

$$
\begin{array}{lll}
119000-39-4 & \mathrm{C}_{32} \mathrm{H}_{18} \quad 402 \quad & 1.722 \\
& & 18.80 \\
& & 10.92 \\
& 3.901
\end{array}
$$

$\begin{array}{ccc}\text { 313-97-3 } \quad \mathrm{C}_{32} \mathrm{H}_{18} \quad 402 \quad 1.873 \\ & & \\ & & 19.30 \\ & & 10.31 \\ & & 3.886\end{array}$

196-46-3

$\mathrm{C}_{32} \mathrm{H}_{18}$

402

2.158

20.93

9.696
3.893 
572

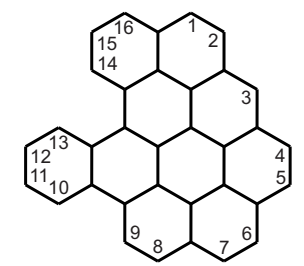

573

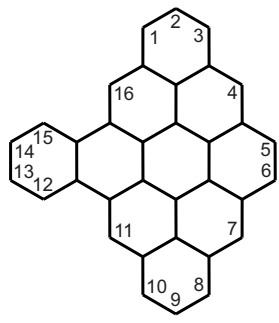

574

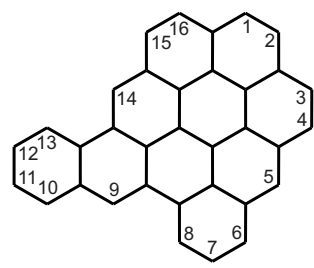

575

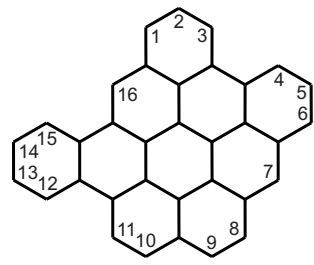

576

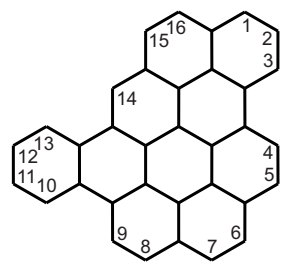

577

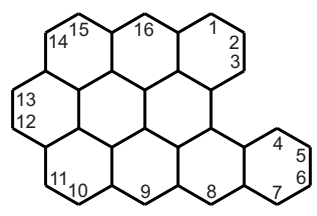

578

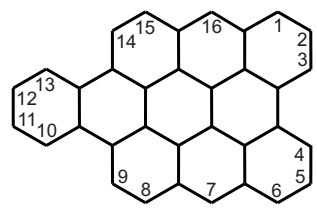

Benzo[p]naphtho[8,1,2-abc]coronene

Tribenzo[a,ef,no]coronene

Benzo[bc]naphtho[3,2,1-ef]coronene

Tribenzo[a,ef,hi]coronene

Benzo[bc]naphtho[1,2,3-ef]coronene

Tribenzo[a, $h i, k]]$ coronene

$\begin{array}{lll}75450-00-9 \quad \mathrm{C}_{34} \mathrm{H}_{16} \quad 424 \quad 1.058 \\ & & 13.82 \\ & 13.06 \\ & 5.000\end{array}$

$128345-68-6$

$\mathrm{C}_{34} \mathrm{H}_{16}$

424

1.113

15.92
14.31

3.897

128345-69-7 $\quad \mathrm{C}_{34} \mathrm{H}_{16} \quad 424 \quad 1.233$

16.01

12.99

3.889

128345-70-0 $\quad \mathrm{C}_{34} \mathrm{H}_{16} \quad 424 \quad 1.235$

16.03

12.03
12.98
3.893

$$
\begin{array}{llll}
\text { Benzo[g]naphtho[8,1,2-abc]coronene } \quad 75449-86-4 & \mathrm{C}_{34} \mathrm{H}_{16} & 424 & 1.241 \\
& & 16.04 \\
12.92 \\
3.888
\end{array}
$$

$\begin{array}{llll}128345-72-2 \quad \mathrm{C}_{34} \mathrm{H}_{16} \quad 424 \quad & 1.296 \\ & & 15.39 \\ & 11.88 \\ & 4.962\end{array}$

$\begin{array}{lll}128345-67-5 \quad \mathrm{C}_{34} \mathrm{H}_{16} \quad 424 \quad \begin{array}{l}1.311 \\ \\ \end{array} & 15.46 \\ & 11.79 \\ & 3.896\end{array}$




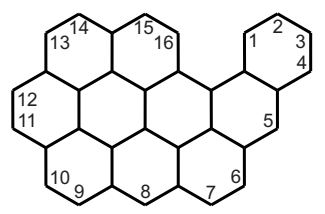

Anthra[9,1,2-abc]coronene

128345-73-3 $\quad \mathrm{C}_{34} \mathrm{H}_{16}$

$424 \quad 1.333$

15.56

11.68

580

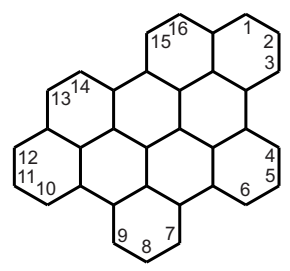

Dibenzo[fg,ij]phenanthro-

$[2,1,10,9,8,7$-pqrstuv $]$ pentaphene

187-94-0 $\quad \mathrm{C}_{34} \mathrm{H}_{16}$

424

1.359

16.00

11.78
3.894

581

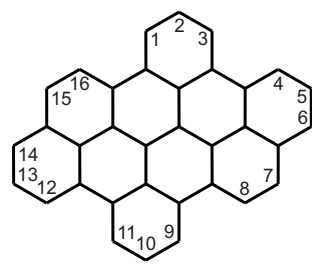

Perylo[3,2,1,12-pqrab]perylene

187-95-1

$\mathrm{C}_{34} \mathrm{H}_{16}$

$424 \quad 1.361$

16.02
11.78
3.893

582

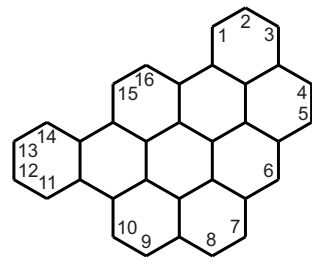

Benzo[ $m]$ naphtho[8,1,2-abc]coronene

75449-99-9

$\mathrm{C}_{34} \mathrm{H}_{16}$

424

1.372

16.10
11.74
3.895

583

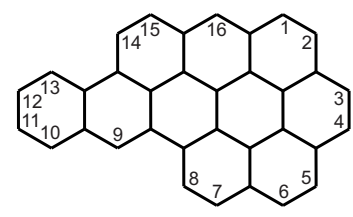

Phenanthro[10,1,2-abc]coronene

75459-01-7

$\mathrm{C}_{34} \mathrm{H}_{16}$

424

1.426

16.71
11.71

3.896

584

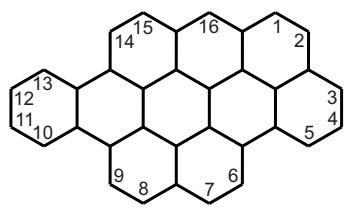

Benzo[J]naphtho[8,1,2-abc]coronene

75459-00-6

$\mathrm{C}_{34} \mathrm{H}_{16}$

424

1.426

16.73 11.73
3.896

585

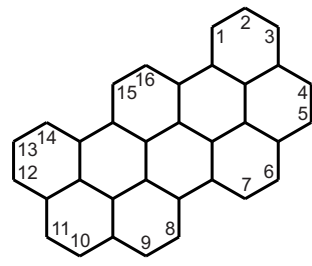

Benzo[pqr]dinaphtho-

[8,1,2-bcd:2', 1', ,'-Imn]perylene

$188-11-4$

$\mathrm{C}_{34} \mathrm{H}_{16}$

424

1.535

16.12

10.50
3.896 
586

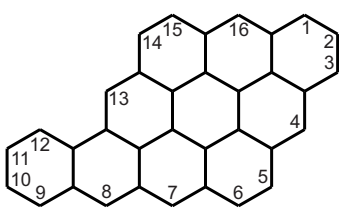

Naphtho[3,2,1,8,7-defgh]pyranthrene

peri-Pentacenopentacene
128345-71-1 $\quad \mathrm{C}_{34} \mathrm{H}_{16}$

424

1.536

18.12
11.80

3.896

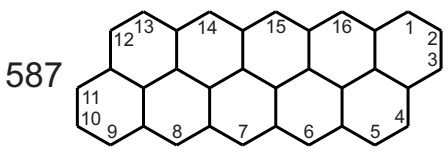

74335-56-1 $\quad \mathrm{C}_{34} \mathrm{H}_{16}$ 
588<smiles></smiles>

589

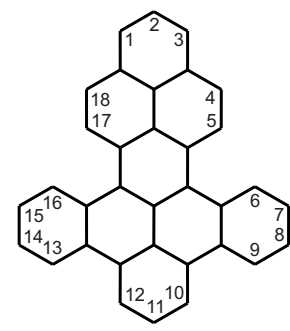

590<smiles>C1CCC2C(C1)CC1C3CCCCC3C3CCC4C5CCCCC5C5CCC6C7CCCCC7C1C6C5C4C23</smiles>

591

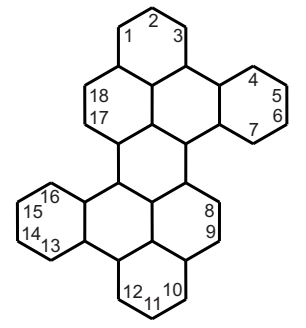

592

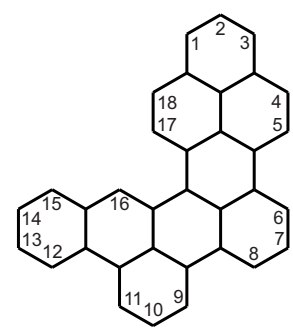

593

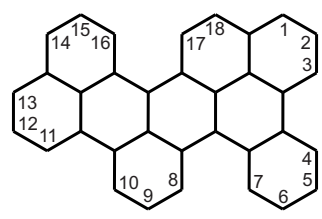

Dibenzo[fg,ij]naphtho[2,1,8-uva]pentaphene

Tetrabenzo[a,cd,j,Im]perylene

191-53-7

$\mathrm{C}_{34} \mathrm{H}_{18}$

426

1.275

14.39 6.281

\section{9-63-4 $\quad \mathrm{C}_{34} \mathrm{H}_{18} \quad 426 \quad 1.125$ \\ 16.08
14.29 3.897}

Dibenzo[j,Im]naphtho[1,8-ab]perylene

93122-98-6

$\mathrm{C}_{34} \mathrm{H}_{18}$

426

1.336

15.50

11.60

594

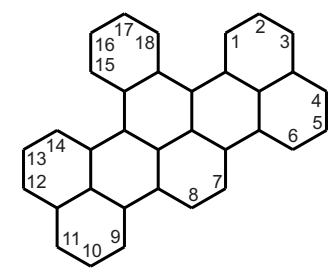

Tetrabenzo[de, $h, k l, r s t]$ pentaphene

$\mathrm{C}_{34} \mathrm{H}_{18}$

426 


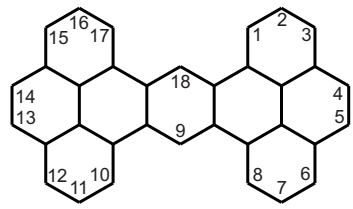

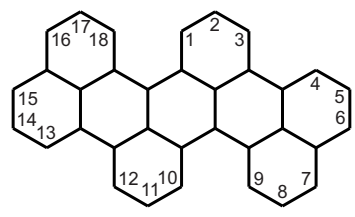

$191-79-7$

$\mathrm{C}_{34} \mathrm{H}_{18}$

426

1.478

16.74
11.33

6.225

597

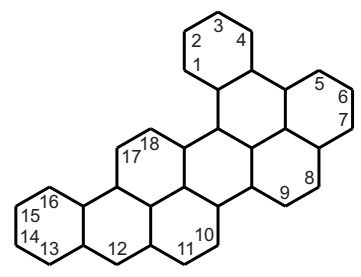

Dibenzo[a,rst]naphtho[8,1,2-cde]pentaphene

$191-46-8$

$\mathrm{C}_{34} \mathrm{H}_{18}$

426

1.543

18.06

5.437

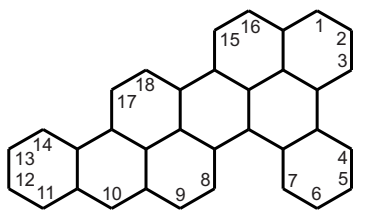

Benzo[rst]phenanthro[1,10,9-cde]pentaphene

190-93-2

$\mathrm{C}_{34} \mathrm{H}_{18}$

426

1.549

18.12

11.70
5.551

599

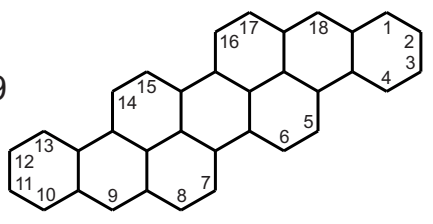

Benzo[rst]phenanthro[10,1,2-cde-] pentaphene

Dinaphtho[1,2,3-cd, 1', 2', 3'-Im]perylene

$\begin{array}{llll}\text { 188-84-1 } \quad \mathrm{C}_{34} \mathrm{H}_{18} \quad 426 \quad & 2.194 \\ & & 20.41 \\ & & 9.302 \\ & 3.896\end{array}$

600<smiles>C1CCC2C(C1)CC1CCC3C(CCC12)C1CCC2C4CCCCC4CC1CCC23</smiles>

Anthra[9,1,2-cde]benzo[rst]pentaphene Dinaphtho[1,2,3-cd,3', 2', 1'-Im]perylene

$188-87-4$

$\mathrm{C}_{34} \mathrm{H}_{18}$

426

2.196

20.42

9.30

3.898

601

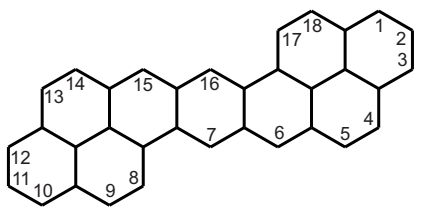

Dinaphtho[2,1,8-jkl:2', 1', 8'-uva]-

123795-83-5 $\quad \mathrm{C}_{34} \mathrm{H}_{18}$

426 
602

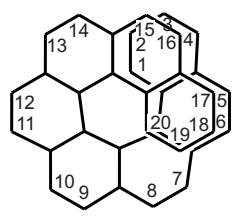

Naphtho[2,1-c:7,8-c]diphenanthrene

Phenanthro[2,1-f]picene

603<smiles></smiles>

604

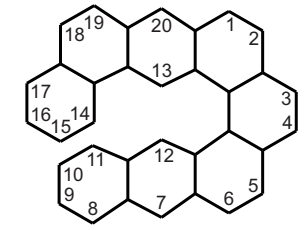

Benzo[]]benzo[2,1-a:3,4-a]dianthracene

122961-15-3

$\mathrm{C}_{34} \mathrm{H}_{20}$

428

1.184

14.32
12.10

7.622<smiles>O=S(=O)(O)c1ccccc1</smiles>

Tetrabenzo[a,c,j,]nnaphthacene

215-95-2

$\mathrm{C}_{34} \mathrm{H}_{20}$

428

1.426

16.78
11.77

3.885

Benzo[6,7]phenanthro[4,3-b]chrysene
119123-34-1 $\quad \mathrm{C}_{34} \mathrm{H}_{20}$

428

1.672

22.15 13.25
3.902

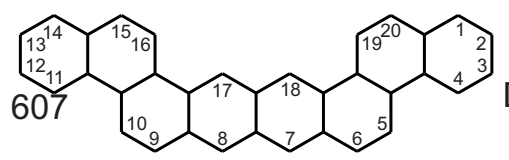

Dinaphtho[2,1-a:1',2'-/]naphthacene

126762-84-3 $\quad \mathrm{C}_{34} \mathrm{H}_{20}$

428

2.270

21.73

9.570<smiles>C1CCC2CCC3C(CCC2C1)CC1CC2CC4C(CCC2CC13)CCC1CC2CCCCC2CCC14</smiles>

Benzo[c]naphtho[2,1-m]pentaphene

119000-41-8 $\quad \mathrm{C}_{34} \mathrm{H}_{20}$

428

2.459

22.63

9.201
3.902

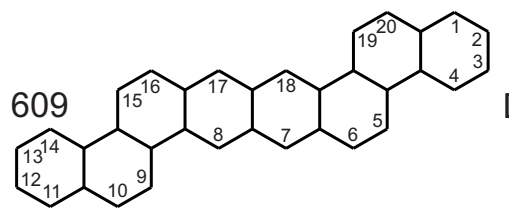

Dinaphtho[2,1-a:2',1'-j]naphthacene

119000-43-0 $\quad \mathrm{C}_{34} \mathrm{H}_{20}$ 
<smiles>C1CCC2CC3CC4CC5CC6CC7CC8CCCCC8CC7CC6CC5CC4CCCCC3CC2C1</smiles>

$\mathrm{C}_{34} \mathrm{H}_{20}$

428

2.544

95.37

3.934<smiles></smiles>

Dinaphtho[1,2-b:1',2'-k]chrysene

214-13-1

$\mathrm{C}_{34} \mathrm{H}_{20}$

428

2.577

22.56

8.754

3.897

612 (134)

258-33-3

$\mathrm{C}_{34} \mathrm{H}_{20}$

428

3.213 
613

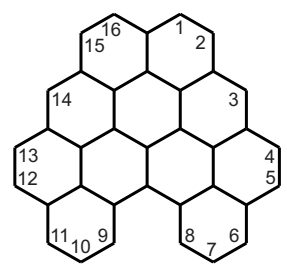

Dinaphtho[8,1,2-abc:2',1',8'-nop]coronene

Dibenzo[ef,no]naphtho[8,1,2-abc]coronene

Anthra[1,9,8-abcd]benzo[hi]coronene

128345-78-8

$\mathrm{C}_{36} \mathrm{H}_{16}$

448

1.160

14.83

12.79

11109

616

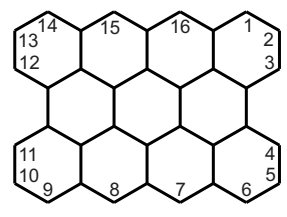

Tetrabenzo[bc,ef, kl,no]coronene

Benzo[ef]phenaleno[9,1,2-abc]coronene

28345-76-6

$\mathrm{C}_{36} \mathrm{H}_{16}$

448

1.225

16.07

3.888

618

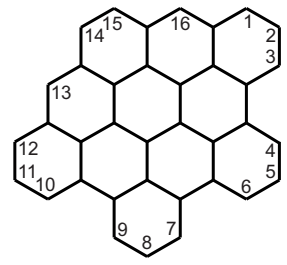

Tetrabenzo[bc,ef,hi,k]coronene

128366-79-0

$\mathrm{C}_{36} \mathrm{H}_{16}$

448

1.226

15.96

13.02

Dinaphtho[8,1,2-abc:2', 1', 8'-efg]coronene
122677-68-3 $\quad \mathrm{C}_{36} \mathrm{H}_{16}$

448

1.229 
620

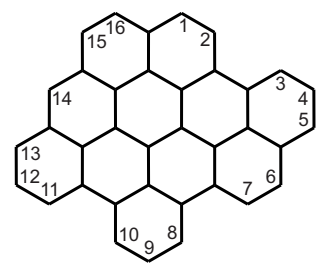

Dibenzo[ef,hi]naphtho[8,1,2-abc]-

128395-03-9

$\mathrm{C}_{36} \mathrm{H}_{16}$

448

1.229

coronene Dibenzo[kl,no]naphtho[8,1,2-abc]-
coronene

128345-75-5 $\quad \mathrm{C}_{36} \mathrm{H}_{16}$

448

1.235

16.04

12.99<smiles>CCCCCCC1CCCC(C)C1</smiles>

622

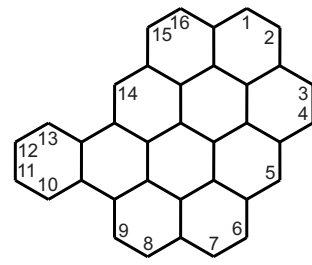

Benz[a]ovalene

75449-88-6 $\quad \mathrm{C}_{36} \mathrm{H}_{16}$ 448

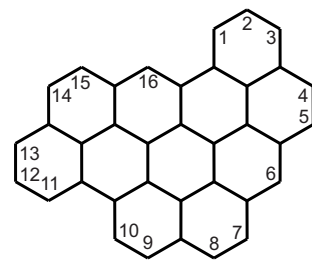

Dinaphtho[8,1,2-abc:8', 1',2'-ghi]coronene

75459-02-8 $\quad \mathrm{C}_{36} \mathrm{H}_{16}$

448

1.267

16.25

3.88

624

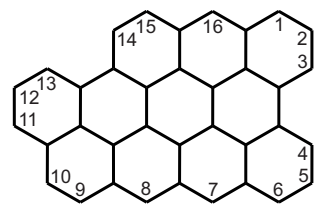

Dibenzo[hi,k/]naphtho[8,1,2-abc]coronene

128345-77-7 $\mathrm{C}_{36} \mathrm{H}_{16}$

448

1.310

15.45
11.79

3.898

625

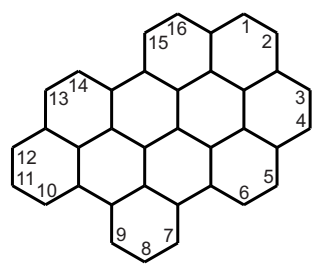

Benz[4,10]anthra[1,9,8-abcd]coronene

117726-83-7 $\quad \mathrm{C}_{36} \mathrm{H}_{16}$

448

1.360

16.02

3.894

626

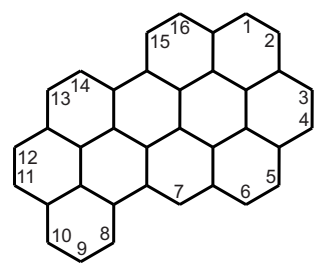

Pyreno[1,10,9-abc]coronene

75459-04-0

$\mathrm{C}_{36} \mathrm{H}_{16}$

448 
627

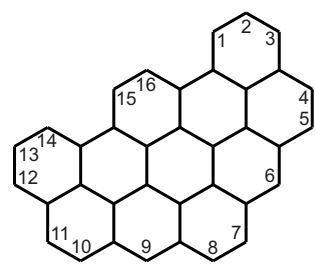
coronene

Pyreno[10,1,2-abc]coronene

Benz[d]ovalene

Dinaphtho[8,1,2-abc:2',1',8'-hij]coronene

Dinaphtho[8,1,2-abc:8',1',2'-jk]coronene

Benzo[qrs]naphtho[3,2,1,8,7-defgh]-

628

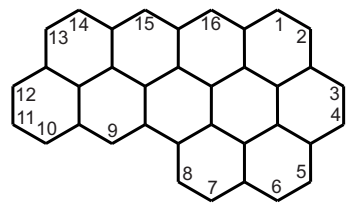

630

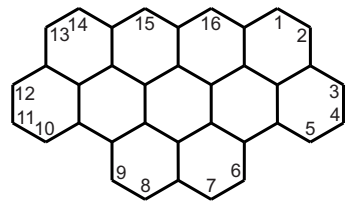

631

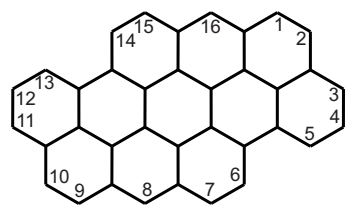

632
Dinaphtho[8,1,2-abc:2', 1', ,'-klm]-

$53086-28-5$

$\mathrm{C}_{36} \mathrm{H}_{16}$

448

1.373

16.11

11.74

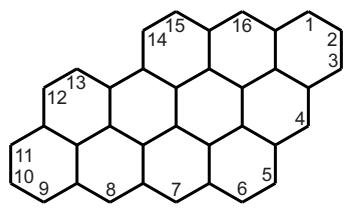

633

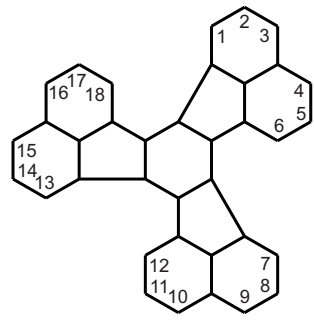

pyranthrene

75449-90-0

$\mathrm{C}_{36} \mathrm{H}_{16}$

448

1.420

16.68

11.75

75449-89-7 $\quad \mathrm{C}_{36} \mathrm{H}_{16}$

448

1.421

16.67
11.73

3.887

$\begin{array}{llll}128345-74-4 & \mathrm{C}_{36} \mathrm{H}_{16} \quad 448 \quad & 1.422 \\ & & & 16.73 \\ & & 11.76 \\ & 3.896\end{array}$

190-47-6

$\mathrm{C}_{36} \mathrm{H}_{16}$

448

1.424

16.71

11.73
3.893

128345-79-9 $\quad \mathrm{C}_{36} \mathrm{H}_{16}$

448

1.530

18.09

11.82

Decayclene

Trinaphthylenebenzene

Benzo[1,2-a,3,4-a',5,6-a']triacenaphthylene 
634

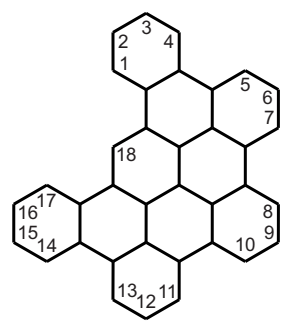

Dibenzo[fg,ij]phenanthro-

$[9,10,1,2,3$-pqrst]pentaphene

$188-00-1$

$\mathrm{C}_{36} \mathrm{H}_{18}$

450

1.126

16.06
14.25
3.891

3.891

635

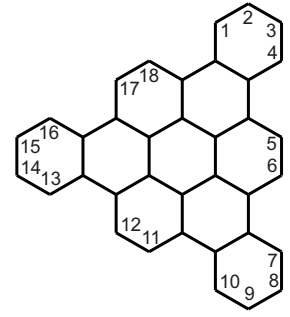

Tribenzo[fgh,pqr,za1b1] trinaphthylene

97269-75-5

$\mathrm{C}_{36} \mathrm{H}_{18}$

450

1.127

16.08

14.27

636

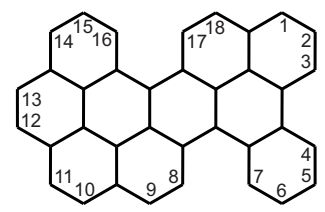

Dibenzo[j,Im] phenanthro-

[5,4,3-abcd] perylene

117726-80-4

$\mathrm{C}_{36} \mathrm{H}_{18}$

450

1.375

15.57
11.32

6.125

637

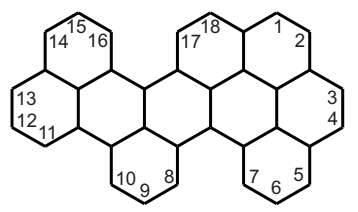

Tribenzo[jk, qr,uv]naphtho-

[2,1,8,7-defg]pentacene

117726-82-6 $\quad \mathrm{C}_{36} \mathrm{H}_{18}$

450

1.483

16.76

11.30
6.070

638

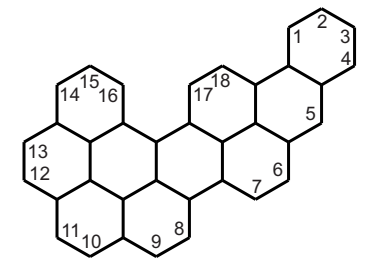

Dibenzo[ $r s, v w x]$ naphtho-

117726-81-5 $\quad \mathrm{C}_{36} \mathrm{H}_{18}$ 450

1.558

[2,1,8,7-k/mn]hexaphene

18.17
11.67

11.67
5.478

639

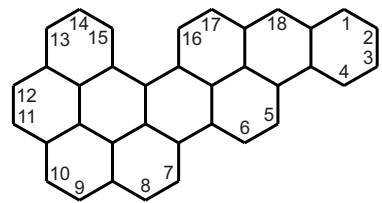

Benzo[rst]pyreno[1,10,9-cde]pentaphene

117740-28-0 $\quad \mathrm{C}_{36} \mathrm{H}_{18}$

450

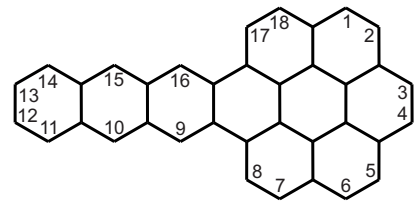

Anthra[2,3-a]coronene

5869-17-0

$\mathrm{C}_{36} \mathrm{H}_{18}$

450 
641

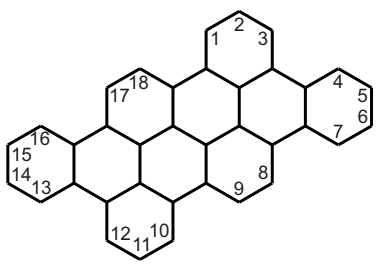

Dibenzo[ij,rst]phenanthro$[9,10,1,2$-defg]pentaphene

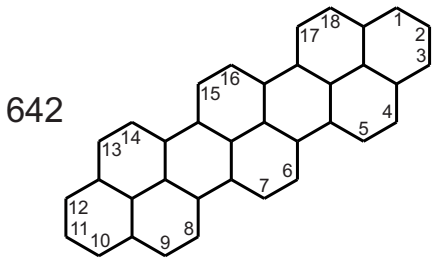

$313-65-5$

$\mathrm{C}_{36} \mathrm{H}_{18}$

450 1.736

18.31

3.894

$\begin{array}{rlll}56181-09-0 & \mathrm{C}_{36} \mathrm{H}_{18} \quad 450 \quad & 2.183 \\ & & & 20.33 \\ & 9.312 \\ & 3.899\end{array}$

Benzo[rst]dinaphtho-

$\left[8,1,2-c d e: 2^{\prime}, 1^{\prime}, 8^{\prime}-k l m\right]$ pentaphene 
643<smiles></smiles><smiles></smiles>

Tetrabenzo[a,e,j,o]perylene

128721-01-7 $\quad \mathrm{C}_{36} \mathrm{H}_{20}$

15.40

14.06

7.324<smiles>C1CCC2CC3C(CC2C1)C1CCCCC1CC1C2CCCCC2C2CC4CCCCC4CC2C31</smiles>

Dinaphtho[3,2,1-fg:3',2', 1'-qr]-

$128721-00-6$

$\mathrm{C}_{36} \mathrm{H}_{20}$

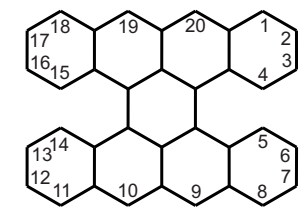<smiles>Clc1ccccc1Br</smiles>

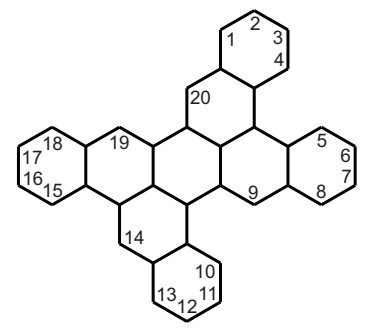

\section{Dinaphtho[1,2,3-fg:3',2', 1'-qr]-
pentacene}

128720-98-9 $\quad \mathrm{C}_{36} \mathrm{H}_{20}$ 452

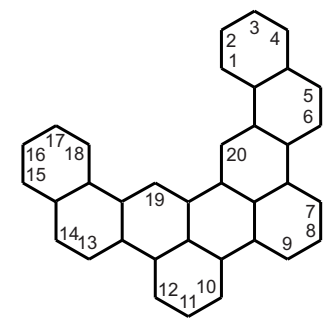

Dinaphtho[1,2-b,2', 1'-n]perylene

$\mathrm{C}_{36} \mathrm{H}_{20}$ 
650<smiles>C1CCC2C(C1)CC1CCCCC1C1C2CC2CCCCC2C2C3CCCCC3CC3CCCCC3C21</smiles>

Dinaphtho[3,2,1-fg:1',2',3'-ij]pentaphene

$128720-99-0$

$\mathrm{C}_{36} \mathrm{H}_{20}$

Dinaphtho[1,2,3-fg:1',2',3'-qr]pentacene

651<smiles>C1=CC2C3CCCCC3CC3CC2C(C1)CC1C3CC2CCCCC2C2C3CCCCC3CC12</smiles>

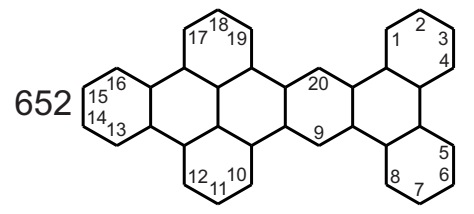

Tetrabenzo[a,c,hi,qr]pentacene

Dinaphtho[1,8-bc:1',8'-mn]picene

653

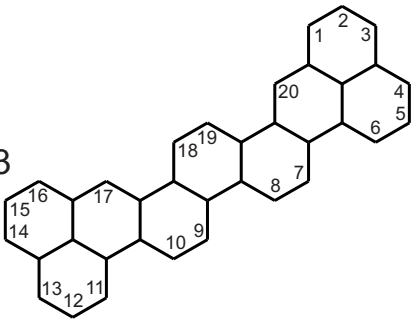

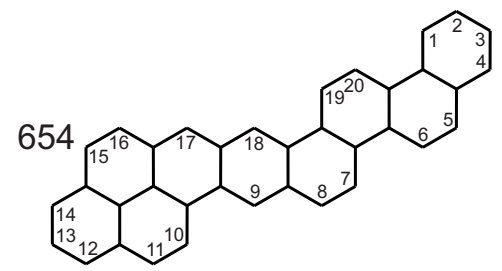

Pyreno[2,1-b]picene

Dibenzo[b,tuv]naphtho[2,1-m]picene

$13354-54-6$

$\mathrm{C}_{36} \mathrm{H}_{20}$

\section{$36474-85-8$}

$\mathrm{C}_{36} \mathrm{H}_{20}$

452

1.317

15.68

11.90
4.969

192-58-5 $\quad \mathrm{C}_{36} \mathrm{H}_{20}$

1.541

17.86
1159

5.279

128721-02-8 $\quad \mathrm{C}_{36} \mathrm{H}_{20}$

$452 \quad 2.224$

20.60

9.265<smiles></smiles>

452 2.436 
Structure

Name
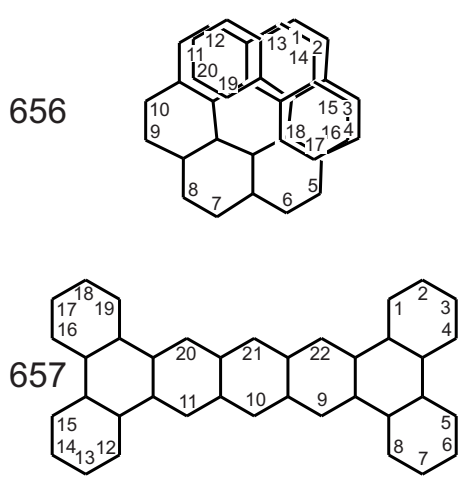

Tetrabenzo[a,c,l,n]pentacene

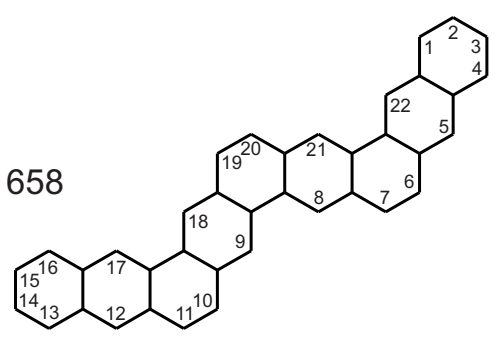

Dinaphtho[2,3-c:2', 3'-m]pentaphene $\quad 133979-16-5 \quad \mathrm{C}_{38} \mathrm{H}_{22}$

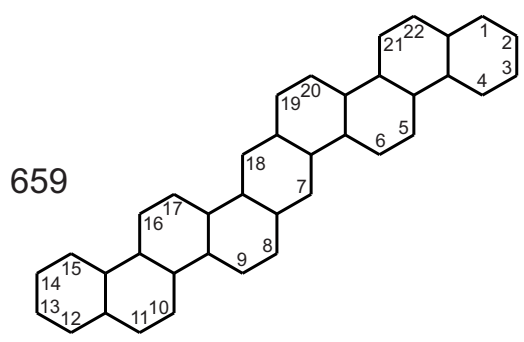

Chryseno[2,1-b]picene

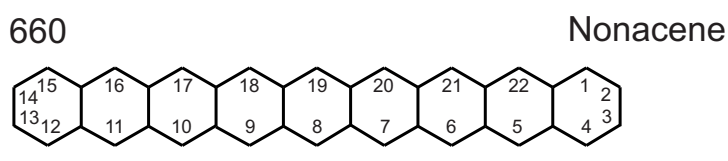

CAS\# Formula MW L/B

20495-14-1 $\quad \mathrm{C}_{38} \mathrm{H}_{22}$

478

1.112

phenanthrene

216-07-9

$\mathrm{C}_{38} \mathrm{H}_{22}$

478

1.637

19.26

3.887

478

2.688

24.80
9.224

9.224
3.904

119000-37-2 $\quad \mathrm{C}_{38} \mathrm{H}_{22}$

478

2.770

24.72
8.927

3.907

258-36-6 $\quad \mathrm{C}_{38} \mathrm{H}_{22}$

478

3.488

26.46
7.585

3.887 